\title{
4 Empirische Untersuchung: Ergebnisse
}

\subsection{Zielsetzung des Kapitels}

Das folgende Kapitel widmet sich der Darstellung und Interpretation der Ergebnisse aus der Analyse. Das erste Teilkapitel (4.2) geht auf die strukturelle Gliederung des Kasusdiasystems ein und konzentriert sich auf die Ergebnisse der Korpusanalyse. Es hat zum Ziel, die abstrakten Kasus, die im gesamten Diasystem angesetzt werden können, herauszuarbeiten und abzubilden, wie diese formal markiert werden. Es bezieht sich sowohl auf die morphologischen Strukturen, d. h. die Typen der Kasusmarkierung, als auch auf die Kontexte, in denen diese abstrakten Kasus distinkt markiert werden. Der Tradition der strukturellen Dialektologie entsprechend (vgl. 2.3.1) zeigt dieses Kapitel damit zunächst einmal die Gemeinsamkeiten der im Untersuchungsgebiet auftretenden Teilsysteme und damit die konstituierenden Faktoren des Kasusdiasystems auf.

Das zweite Teilkapitel (4.3) konzentriert sich auf die geographische Gliederung des untersuchten Diasystems und berücksichtigt dazu sowohl die Daten der Korpusanalyse als auch die aus der Untersuchung der Ortsgrammatiken. Ziel ist es hier, zunächst zu ermitteln, welche der in 2.3.1 für das Diasystem einheitlich beschriebenen Kategorien geographisch gegliedert sind, welche Strukturen der morphologischen Raumgliederung sich in den untersuchten Daten zeigen und inwiefern diese die Annahmen der bisherigen Forschungsliteratur widerspiegeln.

Während sich die ersten beiden Teilkapitel auf die rein paradigmatische Analyse des Kasusdiasystems konzentrieren, gibt das dritte Teilkapitel einen Ausblick in vertiefende syntagmatische Untersuchungen der Korpusdaten.

\subsection{Strukturelle Gliederung des Kasusdiasystems}

Dieses Teilkapitel bildet die strukturelle Gliederung des Kasusdiasystems ab, d. h. die teilsystemübergreifenden Strukturen und Muster, die sich im Kasussystem des Untersuchungsgebiets zeigen. Auf Grundlage der quantitativen Daten der Korpusanalyse werden die abstrakten Kasus, die im Diasystem auf Grundlage der Datenbasis angesetzt werden können, definiert und hinsichtlich ihrer Frequenz sowie ihres Anteils an distinkter Kasusmarkierung hierarchisiert. Im Anschluss widmet sich das Kapitel dem formalen Ausdruck dieser abstrakten Kasus. Es gewährt einen ersten quantitativ basierten Einblick in die morphologischen Strukturen im Diasystem und greift die bereits in 3.3.1 angeführte 
These auf, dass die (distinkte) Kasusmarkierung im (ober)deutschen Diasystem insbesondere durch suppletive Strukturen erfolgt. Die Gliederung der Muster der Kasusmarkierung zeigt einen gewissen Einfluss von Person, Numerus und Genus. Anhand der frequenzbasierten Analyse dieser Muster wird die Dependenz der Kasusdistinktion von eben diesen drei grammatischen Kategorien beschrieben und es wird ermittelt, welche der Kategorien besonders starken Einfluss auf die Kasusmarkierung ausüben. Das Teilkapitel mündet in eine Definition der zentralen Kasusmarker, das heißt der Wortarten, die einen besonders hohen Anteil an distinkten Belegen im Korpus zeigen und der kanonischen Kasusmarkierung damit besonders nahekommen.

\subsubsection{Abstrakte Kasus im Diasystem: eine Hierarchisierung}

Die kurze Übersicht über die diachrone Entwicklung des (ober)deutschen Kasussystems sowie die Einschätzungen von Dal (1971a, 1971b) haben erste Hinweise auf den Erhalt der distinkten Markierung von Nominativ, Akkusativ und Dativ im Diasystem und auf die damit verbundene Hierarchisierung der abstrakten Kasus gegeben, denen hier auf Basis von quantitativen Daten nachgegangen wird. Die Definition der abstrakten Kasus im Diasystem erfolgt gemäß der lateinisch-griechischen Tradition der Kasusmorphologie (vgl. 2.2.2). Die mithilfe des kanonischen Bezugssystems zugeordneten kanonischen Kasus werden dann als abstrakte Kasus angesetzt, wenn sie an einer Stelle im Diasystem distinkt markiert werden, wenn sich demnach mindestens ein distinkter Beleg für den kanonischen Kasus im Korpus findet. Abstrakte Kasus werden hier dementsprechend rein paradigmatisch, folglich ausschließlich durch morphologische Distinktionen bestimmt.

Im Folgenden gebe ich zunächst einen grundlegenden Überblick über die Tokenfrequenz der kanonischen Kasus im untersuchten Korpus. Je höher die Tokenfrequenz eines kanonischen Kasus bzw. der anderen in den folgenden Kapiteln aufgeführten Einheiten im Korpus ist, je mehr Belege also zur Untersuchung zur Verfügung stehen, desto detaillierter und zuverlässiger sind die Erkenntnisse, die sich auf Grundlage der Daten darüber gewinnen lassen. Die Position der Kasus innerhalb der Hierarchisierung wird jedoch nicht anhand ihrer Frequenz, sondern anhand ihres Anteils an distinkten Belegen bestimmt. Je größer der Anteil distinkter Belege eines abstrakten Kasus im Korpus ist, d. h. je häufiger er distinkt markiert wird, desto höher ist die Position dieses Kasus innerhalb der Hierarchie. 
Tab. 7: Frequenz kanonischer Kasus und Anteile ihres distinkten und synkretischen Ausdrucks.

\begin{tabular}{llrrrrr}
\hline kanonischer Kasus & \multicolumn{2}{c}{ Belegmenge } & \multicolumn{3}{c}{ formaler Ausdruck } \\
\cline { 4 - 7 } & & & \multicolumn{2}{c}{ distinkt } & \multicolumn{2}{c}{ synkretisch } \\
\hline Nominativ & 14.556 & $59,9 \%^{60}$ & 6.021 & $41,4 \%$ & 4.876 & $33,5 \%$ \\
\hline Akkusativ & 5.710 & $23,5 \%$ & 729 & $12,8 \%$ & 3.981 & $69,7 \%$ \\
\hline Dativ & 3.997 & $16,4 \%$ & 2.935 & $73,4 \%$ & 556 & $13,9 \%$ \\
\hline Genitiv & 39 & $0,2 \%$ & 11 & $28,2 \%$ & 17 & $44,0 \%$ \\
\hline & $\mathrm{n}=24.305$ & & & & & \\
\hline
\end{tabular}

Tabelle 7 fasst die Menge der Belege zusammen, die den einzelnen kanonischen Kasus in der Analyse zugeordnet worden sind, und listet den jeweiligen Anteil an synkretischen und distinkten Formen auf. Insgesamt wurden im Korpus nach dem in 3.5.1 beschriebenen Vorgehen 24.305 Belege für kasustragende Wortarten ermittelt. Lediglich bei 7 Belegen konnte aufgrund von unvollständigen Teilsätzen kein kanonischer Kasus zugeordnet werden. Bei 5.178 Belegen konnte aufgrund unvollständiger Paradigmen keine zuverlässige Zuteilung von synkretischer oder distinkter Realisierung erfolgen (vgl. 3.5.3).

Mithilfe des in 3.3.3 beschriebenen kanonischen Kasusparadigmas wurden 14.559 Belege dem kanonischen Nominativ, 5.709 Belege dem kanonischen Akkusativ und 3.995 Belege dem kanonischen Dativ zugeteilt. Entgegen der in 2.3.4 formulierten Annahme sind im Korpus jedoch nicht nur diese drei kanonischen Kasus belegt. Es finden sich auch 39 Belege für einen kanonischen Genitiv - ein Umstand, der nicht unbedingt überrascht: Die Annahme, dass in den deutschen Dialekten kein funktionsfähiger synthetischer Genitiv mehr zu finden ist, gründet sich auf Arbeiten (vgl. beispielsweise Shrier 1965, Dal 1971b), die auf rein basisdialektalen Daten basieren und die sich zumeist auf Nennformenparadigmen in Grammatiken stützen (vgl. 2.3.2). Die Daten aus Ruoff (1984) stellen, wie bereits in 3.4.2 ausgeführt, grundsätzlich keine rein basisdialektale Datengrundlage dar,

60 Die Prozentwerte in der Tabelle ergeben sich wie folgt: Die Werte in der Spalte „Belegmenge" führen die absolute Anzahl sowie die Anteile der einzelnen kanonischen Kasus an der Gesamtbelegmenge auf. Nicht aufgeführt sind hier die Belege, denen kein kanonischer Kasus zugeordnet werden konnte. Die Werte in der Spalte „formaler Ausdruck“ führen die Anzahl sowie Anteile an synkretischen und distinkten Belegen an der Belegzahl des jeweiligen kanonischen Kasus auf. Der Anteil der nicht-eindeutigen Belege wird hier nicht mit aufgeführt. 
wodurch gewisse Vertikalisierungstendenzen bezüglich des Genitivgebrauchs, insbesondere in Possessivkonstruktionen, nicht ausgeschlossen sind. Eine solche Erklärung liegt etwa bei Beispiel (7) nahe: Das Transkript wurde in Ruoff (1984: 111) als umgangssprachlich/hochsprachlich klassifiziert (vgl. 6.2.1) und der thematische Kontext der Belege weicht ebenfalls stark von den in den Alltagstexten prototypisch auftretenden Themen (wie beispielsweise Landwirtschaft, Haushalt und Kriegserzählungen) ab.

\section{(7) hier wird also für die gaistige Freihait där Frau, fir die Mitvärantwortung där} Frau gekempft.

,Hier wird also für die geistige Freiheit der Frau, für die Mitverantwortung der Frau gekämpft.

Belege 22648 und 22649, Stuttgart

Insgesamt stammt ein Großteil der Genitivbelege im Korpus (25 Belege, 64\%) aus den beiden standardnächsten Transkripten (Stuttgart und Öhringen_1). Bei den übrigen Transkripten, die Belege für synthetischen Genitiv zeigen, und die nach Ruoff (1984) als dialektaler eingestuft werden können, treten diese Genitivbelege hingegen nur vereinzelt auf. Dies unterstützt die These, dass es sich hierbei nicht um endemische, aber niedrigfrequente Formen, sondern um ein Vertikalisierungsphänomen handelt.

Bezüglich der Tokenfrequenz des kanonischen Genitivs in dieser Analyse lässt sich allerdings feststellen, dass dieser Kasus mit einem Anteil von lediglich 0,2\% im untersuchten Diasystem ein äußerst niedrigfrequentes Phänomen darstellt. Die geringe Belegmenge im Korpus reicht damit für eine vertiefende Analyse - insbesondere zu den Kontexten, in denen der Genitiv auftritt, und damit zu seiner Funktion in verschiedenen Sprechlagen - nicht aus. Sie legt allerdings nahe, dass der synthetische abstrakte Genitiv im Vergleich zu den übrigen Kasus eine marginale Rolle im Diasystem spielt. ${ }^{61}$

Beim Nominativ handelt es sich hingegen um den Kasus, der im Korpus mit einem Anteil von 59,9\% an der Gesamtzahl am häufigsten belegt ist. Ihm folgt der Akkusativ mit einem Anteil von 23,5\% und schließlich der Dativ mit 16,4\%. Für die Analyse bedeutet das zunächst methodisch, dass grundlegend mehr Daten zum kanonischen Nominativ vorhanden sind als zu den obliquen Kasus und darüber somit besonders zuverlässige Aussagen getroffen werden können. Während zum kanonischen Genitiv so wenige Daten vorliegen, dass keine zuverlässigen Aussagen möglich sind, reicht die Datenmenge $\mathrm{zu}$ den beiden anderen kanonischen

61 Eine vollständige Übersicht über alle Belege des kanonischen Genitivs findet sich in Anhang 6.2.2. 
Kasus (Dativ und Akkusativ) jedoch aus, um dort neben allgemeinen Tendenzen auch weitere Phänomene und spezifischere Muster der Kasusmarkierung zu analysieren. Die weitere Analyse konzentriert sich auf die drei ausreichend belegten kanonischen Kasus.

Diese weisen allesamt einen gewissen Anteil an formaler Distinktion auf, können daher als abstrakte Kasus im Diasystem angesetzt werden. Tabelle 7 zeigt zudem, dass der Dativ mit (73,4\%) den größten Anteil an formaler Distinktion und den geringsten Synkretismusanteil besitzt. Auch die Nominativbelege weisen, wenn auch nicht derart ausgeprägt wie beim Dativ, grundlegend häufiger distinkte als synkretische Realisierung auf, während bei den Akkusativbelegen synkretische Belege überwiegen. ${ }^{62}$

Die Daten spiegeln damit quantitativ basiert die Einschätzung aus Dal (1971a, 1971b) wider, die den hochdeutschen Dialektraum als „Dativgebiet“ (Dal 1971a: 189) charakterisiert und damit auf die Prominenz einer distinkten Dativmarkierung im hochdeutschen Diasystem verweist. Die Einschätzung stützt sich auf den starken Erhalt des synthetischen Dativs in hochdeutschen Dialekten. Basierend auf Daten aus Grammatiken arbeitet Dal (1971b: 174-177) verschiedene Distinktionskontexte heraus, die in diesen Systemen für die starke Stellung des Dativs gegenüber Nominativ und Akkusativ verantwortlich sind. Auch in den Korpusdaten zeigen deutlich mehr Kategorien eine Distinktion beim Dativ als eine Distinktion der beiden anderen Kasus: Lediglich beim Personalpronomen der 1. Person Plural (890 Belege) und vereinzelt im Singular (94 Belege) sowie bei wenigen Belegen im Maskulinum findet sich ein Muster mit synkretischem Dativ (N/AD), während etwa das Muster NA/D, das Nominativ-Akkusativ-Synkretismus zeigt, im gesamten Plural der 3. Person, im Femininum und Neutrum dominant ist und beim Maskulinum zumindest häufiger als das Muster N/AD auftritt. ${ }^{63}$

Dal (1971b: 176) führt zudem verschiedene Phänomene an, die illustrieren, dass die Funktion des Dativs im Hochdeutschen die des standardsprachlichen Systems (d. h. die Markierung des indirekten Objekts sowie bestimmter Präpositionalobjekte und adverbialer Bestimmungen) übersteigen. Einige davon, wie beispielsweise der possessive Dativ (z. B. Dr Baur ischt au a Luder, denn dem saine Viecher die fahret au gärn ibers Eck rom, Beleg 9038,

62 Aufgrund der hier noch relativ einfachen Datenstruktur hat sich an dieser Stelle ein einfacher $\chi^{2}$-Test (Pearsons' $\chi^{2}$ ) angeboten, der im Allgemeinen eine starke Assoziation des abstrakten Kasus mit der binären Variable der Kasusdistinktion aufgezeigt hat $(\mathrm{Chi}=3,985.2, \mathrm{df}=3$, p-Wert < 2.2e-16). Die Residualwerte zeigen hier eine starke positive Assoziation von Dativ und Nominativ mit Distinktion (27,7 und 6,7) und von Akkusativ mit Synkretismus (34).

63 Eine ausführliche Übersicht über die Muster der Kasusmarkierung gegliedert nach grammatischen Kategorien findet sich in Tab. 20 und 21. 
Erkenbrechtsweiler/Nürtingen) und weitere Periphrasen, die auf synthetischen Dativformen basieren, sind auch im Korpus belegt.

Die allgemeine Übersicht über die Korpusdaten in Tab. 8 zeigt nun, dass der Dativ nicht nur, wie von Dal (1971a) beschrieben, viele Funktionen und Gebrauchskontexte besitzt und in vielen grammatischen Kategorien distinkt markiert wird, sondern dass eben diese Distinktion in den Daten auch rein quantitativ im Vergleich zum Nominativ und Akkusativ äußerst stark ausgeprägt ist. Nominativ und Akkusativ werden zwar beide im Vergleich zum Dativ anteilig weniger häufig distinkt markiert, sie lassen sich aber ebenfalls hierarchisieren: Der kanonische Nominativ zeigt einen signifikant höheren Anteil an distinkten als an synkretischen Belegen. Beim kanonischen Akkusativ überwiegen hingegen die synkretischen Belege. Aus quantitativer Sicht lässt sich dem Nominativ folglich eine höhere Position als dem Akkusativ in der Hierarchie zuordnen. Diese Distinktionshierarchie der abstrakten Kasus im Diasystem lässt sich also wie folgt beschreiben:

Dativ $>$ Nominativ $>$ Akkusativ

Beachtet man die bisherigen Erkenntnisse zur diachronen Entwicklung (2.2.2.1) und dialektgeographischen Verbreitung (2.3.4) der Muster dieser beiden Kasus, ist dieses Ergebnis erstaunlich: Insbesondere Nominativ und Akkusativ neigen dazu, formal zusammenzufallen und damit in ähnlichen grammatischen Kategorien Synkretismus aufzuweisen. In allen grammatischen Kategorien, in denen der Nominativ synkretisch ist (z. B. im Femininum und Neutrum Singular und im Plural), weist er immer Formengleichheit mit dem Akkusativ auf. Beim Akkusativ ist nur in wenigen grammatischen Kategorien (z. B. beim Reflexivpronomen oder Adjektiven und in bestimmten geographischen Gebieten bei maskulinen Artikelformen, vgl. 2.3.4) zusätzlich mit Formengleichheit mit dem Dativ zu rechnen. Ein solch starker Unterschied im Anteil der distinkten Belege von Nominativ und Akkusativ war daher nicht zu erwarten.

Für die unterschiedliche Hierarchieebene dieser beiden Kasus gehen aus den Korpusdaten drei Ursachen hervor: Die Kategorien, in denen Dativ-Akkusativ-Synkretismus auftritt (die 1. Person Plural sowie Teile der 1. Person Singular und des Maskulinums) nehmen zwar lediglich einen Anteil von 16,7\% der gesamten Muster im Korpus ein, sind damit aber doch für einen gewissen Mehranteil an synkretischen Formen des Akkusativs gegenüber dem Nominativ verantwortlich. Der hohe Anteil an Synkretismus beim Akkusativ ist damit zum Teil dadurch bedingt, dass der Akkusativ je nach grammatischer Kategorie nicht nur Formengleichheit zum Nominativ, sondern auch zum Dativ aufweisen kann. Der Akkusativ ist damit grundsätzlich „,anfälliger“ für Synkretismus als die beiden anderen Kasus, 
die, abgesehen von Mustern des totalen Synkretismus, nur mit jeweils einem anderen Kasus formal zusammenfallen können.

Zudem überwiegt der Anteil der Nominativbelege im Korpus nicht nur generell, sondern auch in Kontexten, in denen grundsätzlich die volle Distinktion N/A/D auftreten kann, in denen es demnach distinkte Nominativ- und Akkusativformen gibt. Die Belege der einzelnen abstrakten Kasus sind folglich nicht homogen über die verschiedenen Kategorien verteilt. In der 1. Person Singular treten etwa 42,6\% der Nominativbelege (ich) auf, während hier lediglich 15,2\% der Akkusativbelege (mich) zu finden sind, was einen Mehranteil distinkter Nominativbelege gegenüber denen des Akkusativs erklärt.

Eben diese Tendenz wird durch ein weiteres Phänomen verstärkt: das frequente Auftreten des Indefinitpronomens man im Korpus. Wie in 3.5.1 bereits angeführt, nimmt man eine Sonderstellung bezüglich der distinkten Nominativmarkierung ein. Es tritt ausschließlich im Nominativ auf, bildet daher keine paradigmatische Reihe. Jeder Beleg von man kann daher als distinkte Nominativmarkierung gewertet werden. man tritt mit 2.094 Belegen (8,4\% der Gesamtbelegzahl) für eine einzelne Wortform recht frequent im Korpus auf ${ }^{64}$ und trägt damit ebenfalls zum größeren Anteil distinkter Formen bei.

Das Diasystem konnte mithilfe der Korpusdaten als Dreikasussystem beschrieben werden, das auf paradigmatischer Ebene Nominativ, Akkusativ und Dativ unterscheidet. Aus typologischer Sicht handelt es sich damit um ein atypisches System: Blake (2001: 158) geht davon aus, dass Dreikasussysteme typischerweise neben einem Nominativ und einem Akkusativ einen Genitiv unterschieden. Die Korpusdaten weisen jedoch aus paradigmatischer Perspektive auf eine marginale Rolle des synthetischen Genitivs im Diasystem hin, dessen Funktionen dort primär durch periphrastische Konstruktionen, die auf Dativformen basieren, übernommen werden. Blakes (2001) Einschätzung basiert jedoch ausschließlich auf einer Analyse synthetischer Kasusdistinktion in verschiedenen Sprachsystemen. Eher analytische Konstruktionen, wie beispielsweise Periphrasen, werden dort nicht berücksichtigt. Auf dieser Grundlage entwirft er eine Kasushierarchie, die wie folgt strukturiert ist:

(9) Nominativ $>$ Akkusativ/Ergativ $>$ Genitiv $>$ Dativ $>$ Lokativ $>$ Ablativ/Instrumental $>$ andere

(Blake 2001: 157)

64 Eine mögliche Ursache für die relativ hohe Frequenz des Indefinitpronomens man in den oberdeutschen Daten deutet sich mit dem in Kasper/Werth (2015: 364-365) dargestellten Phänomen der Agens-Defokussierung an, bei der dieses Indefinitpronomen im Speziellen eine zentrale Rolle spielt. 
Sprachen, die grundsätzlich Kasusdistinktion aufweisen, können abhängig davon, welcher Kasus dort auf niedrigster Ebene angesetzt werden kann, an einer bestimmten Position auf der Hierarchie verortet werden (Blake 2001: 157-158). Das hier untersuchte Kasusdiasystem muss an der Position des Dativs verortet werden, da dieser im Diasystem synthetisch markiert wird und die unterste Position der Kasus in der Hierarchie einnimmt. Die Logik der Hierarchie besagt nun, dass das Diasystem diesen Kasus und zudem je einen Kasus aus jeder höheren Position besitzt (d. h. einen Nominativ, einen Akkusativ oder Ergativ, einen Genitiv und eben einen Dativ) (vgl. Blake 2001: 158).

Der bereits über die gesamte Entwicklung des hochdeutschen Systems zu beobachtende Verlust des morphologischen Genitivs lässt sich durch Blakes (2001) Hierarchie also nicht greifen. Obwohl Blake (2001: 159) lediglich das standarddeutsche Vierkasussystem zur Illustration seiner Kasushierarchie heranzieht - wo es sich durch seinen noch relativ starken morphologischen Genitiv problemlos eingliedern lässt - scheint ihm dieses Problem durchaus bewusst zu sein. Er merkt an: „Some Pama-Nyungan languages lack a genitive, the possessor function being expressed via dative [. . .]" (Blake 2001: 160) und beschreibt damit ein Kasussystem, das dem hier untersuchten Diasystem ähnlich zu sein scheint. Diese sowie einige weitere Ausnahmen aus der Hierarchie (vgl. Blake 2001: 158-162 und Mal'čukov/Spencer 2009b: 653) verdeutlichen, dass Blakes (2001) Hierarchie zwar Vorhersagen für einen Großteil der analysierten Sprachsysteme trifft, dass sie jedoch keinen Anspruch auf Allgemeingültigkeit erhebt. Systeme wie das hier beschriebene oberdeutsche Diasystem werden dadurch zwar als atypisch klassifiziert, nicht aber vollkommen ausgeschlossen.

Ein weiterer Aspekt, den Blake (2001: 158) anhand seiner Hierarchie aufzeigt, beschreibt die abstrakten Kasus im untersuchten Diasystem hingegen sehr treffend. Er besagt, dass der Kasus auf der niedrigsten Position besonders viele Funktionen im System ausfüllt: „[. . . ] i. e. it will be a kind of ,elsewhere case““ (Blake 2001: 158). Dies ist ein Aspekt, der die oben bereits mit Dal (1971a) angeklungene Erklärung für die starke formale Distinktion des Dativs im Diasystem aufgreift. Der Dativ nimmt im Diasystem die niedrigste Position auf Blakes (2001) Kasushierarchie ein und hat ein im Diasystem unübertroffen großes Funktionsspektrum. Neben dem Ausdruck des indirekten Objekts wird der Dativ in periphrastischen Konstruktionen zum Ausdruck diverser peripherer Kasusfunktionen genutzt, für die im Diasystem auf paradigmatischer Ebene keine eigene Form (mehr) angesetzt wird, beispielsweise ehemalige Genitiv- (z. B. und des ischt em Schitza au sai Hauptaufgab, Beleg 9056, Erkenbrechtsweiler/Nürtingen) oder Instrumental- (z. B. alles friher hat m'r's mit d'r Sichel noch gschnitta, Beleg 239, Ettenhausen/Crailsheim) und Ortskontexte (d. h. einen Großteil der Proto-Location) 
(z. B. wärklich de sim-mr halt im Garta, Beleg 835, Michelfeld/Schwäbisch Hall). Diese Funktionen werden durch den Dativ größtenteils distinkt markiert. Lediglich bei der Kernkasusfunktion, d. h. dem Ausdruck des indirekten Objekts, neigt der Dativ signifikant häufig zum Synkretismus. ${ }^{65}$ Zwar füllt auch der Akkusativ neben dem Ausdruck des direkten Objekts ebenfalls einzelne, aber verglichen mit dem Dativ deutlich weniger periphere Kasusfunktionen aus. Akkusativperiphrasen drücken den übrigen, nicht durch Dativperiphrasen markierten Bereich der Proto-Location aus (z. B. Richtungsweisungen wie z. B. on dann is er in d' Mihl komma zum gärwa, Beleg 192, Bieringen/ Künzelsau). Im Gegensatz zur sonst starken Tendenz zum Synkretismus neigt der Akkusativ beim Ausdruck dieser Funktionen jedoch signifikant häufig zur Distinktion, ${ }^{66}$ was bei den peripheren Kasusfunktionen zu einer insgesamt starken Distinktion sowohl durch den Dativ als auch durch den Akkusativ - führt. Der Nominativ trägt hingegen neben dem Ausdruck des Prädikativums (z. B. Ha ja, des is a frihrer Schäfer, Beleg 2376, Wildenstein/Crailsheim) ausschließlich Kernfunktionen (vgl. auch 2.2.2.1).

Hier treffen die Vorhersagen der Kasushierarchie für das Diasystem also zu und liefern damit einen Erklärungsansatz dafür, weshalb der Dativ, der mit lediglich 16,4\% die niedrigste Tokenfrequenz der anzusetzenden abstrakten Kasus aufweist, die höchste Distinktionsquote besitzt: Im Fall von Synkretismus können hier zahlreiche Funktionen nicht mehr distinkt markiert werden. Beim Nominativ sind es zwar deutlich weniger, aber hochfrequente Kernkasusfunktionen, weswegen auch hier Synkretismus große Auswirkungen zeigt. Die Hypothese aus 3.3.6, dass sich der Einfluss von Frequenz auf Kasusmarkierung möglicherweise direkt anhand der Frequenz abstrakter Kasus ermitteln lässt, kann insbesondere aufgrund der vergleichsweise niedrigen Frequenz des Dativs jedoch nicht bestätigt werden.

65 Ausgewertet wurde eine Kreuztabelle mit den jeweils binären Variablen, Kernkasus (IO/ NICHT-IO) und Kasusdistinktion (DISTINKT/SYNKRETISCH) (274 distinkte und 65 synkretische IO-, 2661 distinkte und 491 synkretische NICHT-IO-Belege), mit einem Pearsons' $\chi^{2}$-Test (Chi = 2,6944, $\mathrm{df}=1$, $\mathrm{p}$-Wert $=0,1007$ ). Die Residualwerte zeigen eine positive Assoziation von IO und synkretischen Dativbelegen $(1,5)$.

66 Ausgewertet wurde analog eine Kreuztabelle mit den jeweils binären Variablen, Kernkasus (DO/NICHT-DO) und Kasusdistinktion (DISTINKT/SYNKRETISCH) (505 distinkte und 2971 synkretische DO-, 224 distinkte und 1010 synkretische NICHT-DO-Belege), mit einem Pearsons' $\chi^{2}$-Test $(\mathrm{Chi}=21.169, \mathrm{df}=1, \mathrm{p}$-Wert $=4.205 \mathrm{e}-06)$. Die Residualwerte zeigen eine positive Assoziation von NicHT-DO und distinkten Akkusativbelegen $(43,9)$. 


\subsubsection{Morphologische Strukturen: Typen der Kasusmarkierung ${ }^{67}$}

Dieses Teilkapitel widmet sich der morphologischen Struktur, die im untersuchten Diasystem für den distinkten Ausdruck der drei abstrakten Kasus verantwortlich ist, und damit den Typen der Kasusmarkierung. Diese wurden in den (ober)deutschen Dialekten bislang noch nicht gezielt und umfassend untersucht. Ein Blick in typologisch orientierte Arbeiten lässt hier jedoch Rückschlüsse auf die morphologische Struktur kasustragender Wortarten zu. Howe (1996: 70) sieht den Grund für den starken Erhalt distinkter morphologischer Markierung bei Personalpronomen (in den germanischen Sprachen im Allgemeinen) in deren suppletiver Struktur. Er argumentiert, dass suppletive Formen (beispielweise er/ihn/ihm) aus Portmanteaumorphemen bestehen und damit von einem Synkretismus gleich mehrere Funktionen betroffen wären, womit sich die starke Tendenz zur Distinktion gegenüber affigierten Formen, wo das Affix weniger Funktionen bündelt (beispielsweise meine/meine/meiner), erklären ließe. Zudem beobachtet Howe (1996) in den von ihm analysierten germanischen Pronominalparadigmen einen geringeren Einfluss von phonologisch determinierter Formennivellierung auf suppletive Formen. Auf die Entwicklung des (ober)deutschen Kasussystems (2.2.2.1) bezogen wäre etwa ein verminderter Einfluss der Nebensilbenabschwächung auf suppletive Paradigmen (die eher auf einsilbigen Formen basieren) zu vermuten. Mit Howe (1996: 70-71) lässt sich folglich ein grundlegender Zusammenhang zwischen Suppletion und distinkter Kasusmarkierung bei Pronomen herstellen. Dieser kann zwar noch nicht explizit auf weitere kasustragende Wortarten bezogen werden, dennoch lässt Howes (1996) Argumentation vermuten, dass Suppletion und die damit verbundene Kürze (und daher knappe morphophonologische Oberflächenstruktur) auch bei anderen Wortarten mit Kasusdistinktion assoziiert ist.

Für das untersuchte Diasystem ist daher die These zu prüfen, dass diejenigen Wortarten, die eher zu suppletiven Strukturen neigen (wie beispielsweise Personalpronomen), stärker zur distinkten Kasusmarkierung beitragen und damit kanonischere Kasusmarker darstellen als Wortarten, die eher von affigierten Strukturen geprägt sind (wie beispielsweise Adjektive). Diese These baut auf verschiedene Grundannahmen auf, die sich in der Gliederung der folgenden

67 Die Fragestellung, der im folgenden Kapitel nachgegangen wird, wurde bereits in ähnlicher Form und auf Grundlage eines vorläufigen Datensatzes der hier vorgestellten Korpusanalyse in Ellsäßer (2019) aufgegriffen. Gewisse methodische sowie theoretische Überschneidungspunkte mit dieser Arbeit lassen sich daher nicht vermeiden. Dieses Kapitel setzt allerdings andere Schwerpunkte als die bereits erschienene Arbeit, die sich eher mit der theoretischen Modellierung von Typen der Kasusmarkierung auseinandersetzt, und nutzt einen überarbeiteten Datensatz. 
Datenpräsentation und -analyse widerspiegeln: Die verschiedenen kasustragenden Wortarten, die im untersuchten Diasystem aufgrund der Kombination aus nominaler und konkordialer Kasusmarkierung auftreten (vgl. 2.2.2.2), zeigen auf morphologischer Ebene unterschiedliche Suppletionsgrade und damit verschiedene Typen der Kasusmarkierung. Diese verschiedenen Typen, so die Annahme, variieren in ihrem jeweiligen Anteil an distinkter und synkretischer Kasusmarkierung; wobei eher suppletive Typen im Vergleich zu eher affigierten einen höheren Anteil an distinkten Belegen aufweisen und damit die kanonischeren Kasusmarker im Diasystem darstellen.

Im Folgenden werden zunächst rein quantitativ die Anteile der einzelnen kasustragenden Wortarten im Korpus aufgeführt. Diese Wortarten werden dann auf Basis der oben erwähnten Abstraktionen verschiedenen Suppletionsgraden zugeteilt. Auf diese Weise lassen sich die jeweiligen Typen der Kasusmarkierung herausarbeiten. Schließlich werden die Wortarten auf ihren jeweiligen Anteil an synkretischen und distinkten Belegen hin analysiert, wodurch ein möglicher Zusammenhang zwischen dem Typus der Kasusmarkierung und dem Grad der Distinktion untersucht werden kann.

Tabelle 8 zeigt einen Überblick über die hier analysierten kasustragenden Wortarten und deren anteilige Belegmenge im Korpus. Hier sind einige Besonderheiten der Analyse zu erkennen: Wie bereits in 3.5.1 ausgeführt, wurde das Indefinitpronomen man hier nicht in die allgemeine Kategorie der Indefinitpronomen aufgenommen. Es wird in Tab. 8 zwar der Vollständigkeit halber mit angeführt; da es keine paradigmatische Reihe bildet und damit aus der Perspektive der Kategorie Kasus ausschließlich Nominativdistinktion markiert, ${ }^{68}$ wird es bezüglich der Typen der Kasusmarkierung zunächst nicht weiter analysiert. Ähnlich verhält es sich mit dem Reflexivpronomen. Da es ebenfalls keine vollständige paradigmatische Reihe bildet, ${ }^{69}$ wird es in der Tabelle zwar mit aufgeführt, aber nicht in die Untersuchung der Typen der Kasusmarkierung aufgenommen.

68 Man könnte hier auch argumentieren, dass es sich bei Formen des Indefinitpronomens wie einen und einem um Dativ- und Akkusativformen von man handelt. Behaghel (1923: 399 und 412413) führt beispielsweise eine Verbindung zwischen diesen Formen auf, die jedoch synchron nicht mehr transparent ist. Zudem sind im Korpus auch Belege für die Form einer (z. B. Beleg 10988 aner, Königshofen/Dinkelsbühl) vorhanden, die hier eher als entsprechende Nominativform der oben angeführten Formen des Indefinitpronomens angesehen werden kann. Es wäre auch ein Paradigma denkbar, in dem die Nominativ-Singular-Zelle durch zwei verschiedene Formen (man und einer) besetzt ist (in dem also Overabundance vorliegt). Jedoch wäre auch in einem solchen Paradigma der spezielle Status, den die Form man in der Nominativdistinktion einnimmt, hervorzuheben.

69 Das Reflexivpronomen kann keinen abstrakten Nominativ, sondern nur Dativ und Akkusativ ausdrücken. Dort können zwar gewisse Muster der Kasusmarkierung - insbesondere Synkretis- 
Tab. 8: Anteile der analysierten kasustragenden Wortarten an der Gesamtbelegmenge im Korpus.

\begin{tabular}{llc}
\hline Wortart $^{70}$ & \multicolumn{2}{c}{ Belegmenge } \\
\hline Personalpronomen & 7.627 & $31,4 \%{ }^{71}$ \\
\hline Definitartikel & 6.506 & $26,8 \%$ \\
\hline Demonstrativpronomen & 3.222 & $13,3 \%$ \\
\hline man & 2.094 & $8,6 \%$ \\
\hline Indefinitartikel & 1.681 & $6,9 \%$ \\
\hline Adjektiv & \\
\hline Possessivpronomen bzw. - determinierer & 1.531 & $6,3 \%$ \\
\hline Indefinitpronomen & 746 & $3,2 \%$ \\
\hline Reflexivpronomen & 622 & $2,6 \%$ \\
\hline & 275 & $1,1 \%$ \\
\hline
\end{tabular}

Die Tabelle zeigt zunächst, dass insbesondere Personalpronomen, gefolgt von Definitartikeln, den größten Teil der Belege kasustragender Wortarten im Korpus ausmachen. Den drittgrößten Anteil nehmen die Demonstrativpronomen ein. Wie bereits bei der Beschreibung der Belegmengen kanonischer Kasus argumentiert, bedeutet das zunächst rein methodologisch, dass sich in der folgenden Analyse weit zuverlässigere Aussagen über Strukturen und Muster bei diesen Wortarten treffen lassen als über die weniger frequent belegten Wortarten; schlicht deshalb, weil sich die Abstraktion der Typen der Kasusmarkierung und die damit verbundenen Analysen hier auf eine größere Datenmenge stützen können. Das Indefinitpronomen man ist nicht derart häufig belegt wie noch das Demonstrativpronomen. Noch seltener sind Adjektive und Indefinitartikel sowie (die übrigen) Indefinit- und Possessivpronomen.

mus - untersucht werden. Es lässt sich aber keine Abstraktion der morphologischen Struktur über die ganze paradigmatische Reihe hinweg erstellen.

70 Die relativen Häufigkeiten der Wortarten im Korpus ähneln nicht zufällig denen in den Daten des Standardwerks zur Wortfrequenz in gesprochener Sprache, Ruoff (1981: 20). Ruoff (1984) bildet ein Teilkorpus der in Ruoff (1981) genutzten Daten.

71 Die Prozentwerte beziehen sich auf die Anteile der jeweiligen Wortarten an der Gesamtzahl der Belege.

72 In das Korpus wurden ausschließlich attributive Adjektive aufgenommen. An dieser Stelle der Analyse wird nicht zwischen starken und schwachen Adjektiven unterschieden. Ein Ausblick auf die Differenzierung starker und schwacher Adjektive erfolgt in 4.4. 
Bevor im nächsten Schritt die morphologischen Strukturen dieser Wortformen kategorisiert werden, werden kurz die theoretischen Hintergründe der Zuteilung von Affigierung und Suppletion beleuchtet und mögliche weitere methodische Zugänge aufgezeigt. Wie bereits an verschiedenen Stellen (vgl. 2.2.2.2 und 3.3.1) angeklungen ist, wird die morphologische Struktur der Wortarten in dieser Arbeit nicht als binäres Phänomen aufgefasst, sondern vielmehr angelehnt an primär sprachtyplogisch ausgerichtete (Mel'čuk 1994, Corbett 2007, Hippisley et al. 2004, und Siewierska/Bakker 2013), aber auch speziell auf dem Deutschen basierende Arbeiten (vgl. z. B. Werner 1987, Nübling 1999 und Dammel 2008) als skalares Phänomen begriffen, das sich zwischen den Polen der transparenten Affigierung und der totalen Suppletion bewegt.

Eine frühe Darstellung einer solchen Skala stammt von Werner (1987). Unter Bezugnahme auf den Verlauf der diachronen Entwicklung von verbalen Flexionsformen unterscheidet er beispielsweise zwischen Affigierung ohne und mit Stammvarianten, Wurzelflexion und schwacher beziehungsweise starker Flexion (Werner 1987: 300) und legt mit dieser vorläufigen Skala eine Grundlage, die auch zur synchronen Typologisierung verschiedener Strukturen innerhalb eines Diasystems herangezogen werden kann. In einem kurzen Exkurs bezieht er diese größtenteils auf Beobachtungen zur Verbalmorphologie basierende Skala sogar auf die Kasusmarkierung beim Personalpronomen (Werner 1987: 311).

Corbett (2007) nähert sich der Gliederung des Bereichs zwischen Affigierung und Suppletion aus der Perspektive des oben (3.3.3) bereits eingeführten Ansatzes der kanonischen Typologie. Das von ihm definierte kanonische Ideal eines Flexionsparadigmas baut grundlegend auf Affigierung auf. Ziel dieses kanonischen Flexionsparadigmas ist es, zum einen alle Zellen eines Lexems über dieselbe Stammform als diesem Lexem eindeutig zugehörig zu markieren, zum anderen die einzelnen Ausprägungen einer grammatischen Kategorie, wie beispielsweise Kasus, über Flexionsaffixe maximal zu distinguieren. Suppletion stellt in der Arbeit von Corbett (2007) ein nicht-kanonisches Muster dar, da die Fragestellung dort vorsieht, die Formen des Paradigmas eines Lexems nach außen hin als solche eindeutig abzugrenzen (vgl. Corbett 2007: 9). Ähnlich, wie sie in dieser Arbeit für die Bestimmung von Distinktionsgraden genutzt wird, setzt Corbett (2007) die kanonische Typologie ein, um u. a. die Skala zwischen Affigierung und Suppletion zu operationalisieren und den Suppletionsgrad quantitativ zu ermitteln.

Dabei baut er auf der Suppletionsdefinition nach Mel'čuk (1994: 358) auf, wonach Suppletion als eine Form der Beziehung zwischen zwei linguistischen Zeichen - in dieser Arbeit etwa zwei kasustragenden Wortformen - definiert ist. Diese Beziehung wird als Suppletion definiert, wenn erstens die semantische Differenz der Formen maximal regulär ist; wenn es sich im System folglich um eine gramma- 
tische Differenz handelt - hier beispielsweise, wenn sich die beiden Wortformen in unterschiedlichen Zellen einer paradigmatischen Reihe befinden - und zweitens die formale Differenz, d. h. die morphophonologische Oberfläche der beiden Wortformen, maximal unterschiedlich ist (vgl. Mel'čuk 1994: 358 und Corbett 2007: 11). Ausgehend von seiner Definition des maximal nicht-kanonischen Pols beschreibt Corbett (2007: 15-16) nun verschiedene Kriterien, nach denen Belege kategorisiert werden können. Er nennt hier unter anderem die Transparenz der Wortform (je transparenter die Grenze von Stamm und Affix, desto weniger suppletiv ist die Wortform), die Überschneidungen der morphophonologischen Oberfläche (bei teilweiser Überschneidung der morphophonologischen Oberfläche zwischen den Wortarten spricht er von partieller Suppletion; gibt es keine Überschneidung, spricht er von totaler Suppletion) sowie overter beziehungsweise nicht-overter Markierung der relevanten grammatischen Kategorie (nicht-realisierte Elemente gelten hier als suppletiver als realisierte Elemente) (Corbett 2007: 15-16).

Bezogen auf die Fragestellung und methodische Ausrichtung dieser Arbeit können hier gewisse Einschränkungen bezüglich dieser Kategorien getroffen werden. Das Kriterium, das eine Unterscheidung zwischen overter und nichtoverter Markierung einer grammatischen Kategorie vorsieht, wird hier aufgrund der korpuslinguistischen Vorgehensweise nicht in die Skalierung der Suppletionsgrade integriert. Inwiefern eine Kategorie Nullrealisierung aufweist, lässt sich auf Basis der Datengrundlage sowie der wortbasierten Ausrichtung der Analyse nicht bestimmen. Bezüglich der ersten groben Skalierung unter Einbezug der beiden übrigen Kriterien sowie der deutschen Terminologie orientiere ich mich in dieser Arbeit an dem Teil des Irregularitätskontinuums aus dem Überblicksartikel von Dammel (2008: 26), der die alternierenden Formen als Bezugsebene hat und die beiden übrigen Kriterien von Corbett (2007: 15-16) in einer Skala vereint, wobei der Schwerpunkt der Skala auf der Seite der irregulären Formen, d.h. auf der Ausdifferenzierung eher suppletiver Strukturen, liegt (vgl. Dammel 2008: 3-26). Angelehnt daran lässt sich hier zunächst die Skala in Abb. 17 ansetzen, deren einzelne Grade nicht als vollständig getrennte Einheiten, sondern vielmehr als Stufen eines Kontinuums definiert sind.

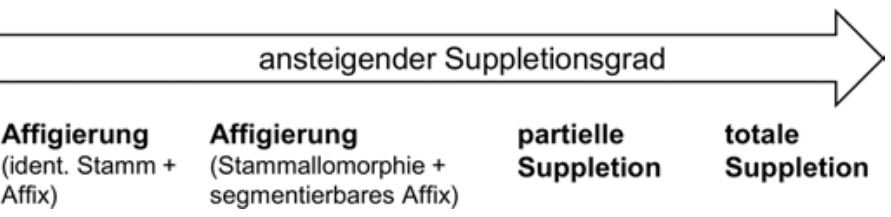

Abb. 17: Suppletionsgrade bei verbalen Kategorien, angelehnt an Dammel (2008: 26). 
Da sich die Arbeit von Dammel (2008), ebenso wie die meisten anderen Arbeiten, die sich mit Suppletion im deutschen Sprachsystem beschäftigen (z. B. Nübling 1999), jedoch empirisch auf verbalmorphologische Flexion stützt, ist die direkte Übertragbarkeit dieser Skala auf die kasusmorphologischen Daten im Verlauf der Analyse kritisch zu hinterfragen. So ist etwa nicht anzunehmen, dass die insgesamt recht kurzen kasusmarkierenden Wortarten als nominale Kategorien zu Stammallomorphie wie in der 2. Stufe in Abb. 17 neigen.

Ebenfalls auf dem kanonischen Ansatz von Corbett (2007) aufbauend beschreiben Siewierska/Bakker (2013: 359-365) ein Vorgehen zur methodischen Umsetzung einer Klassifikation von verschiedenen Suppletionsgraden anhand eines sprachtypologischen Samples von Personalpronomen, das hier exemplarisch angeführt wird. Empirisch zuverlässig und auf große Datenmengen einheitlich anzuwenden wäre hier die von Siewierska/Bakker (2013: 361-365) ausgeführte Berechnung der Levenshtein-Distanz ${ }^{73}$ zwischen zwei Wortformen in verschiedenen Zellen einer paradigmatischen Reihe. Die LevenshteinDistanz quantifiziert im Allgemeinen die Veränderungen, die notwendig sind, um eine Form A in eine Form B umzuwandeln. Dabei würde hier die absolute Menge der morphophonologischen Prozesse berechnet werden, die notwendig sind, um die Wortform der ersten Zelle der der zweiten Zelle anzupassen. Siewierska/Bakker (2013: 364) entwickeln nun wiederum Grenzwerte der Levenshtein-Distanzen, die z. B. die Grenzen von partieller und totaler Suppletion markieren.

Aufgrund ihrer empirischen Exaktheit und mit Blick auf die große Datenmenge, die es in einer Korpusanalyse zu bearbeiten gilt, wäre diese Methode optimal, um die Typen der Kasusmarkierung in den einzelnen kasustragenden Wortarten exakt zu bestimmen. Allerdings sind die notwendigen Voraussetzungen für ein solches Vorgehen beim jetzigen Forschungsstand und auf Basis der Daten in Ruoff (1984) nicht gegeben: Erstens gibt es bislang noch zu wenige Erkenntnisse zu möglicherweise auftretenden Typen der Kasusmarkierung in den unterschiedlichen kasustragenden Wortarten in den dialektalen Systemen des Untersuchungsgebietes. Es gibt also keinerlei Vorwissen, auf das für die Definition von entsprechenden Grenzwerten in der Levenshtein-Distanz zurückgegriffen werden könnte. Zweitens bietet die spezielle Umschrift in Ruoff (1984) bedingt sowohl durch die Variation zwischen den einzelnen

73 In Siewierska/Bakker (2013: 361) wird nicht die Levenshtein-Distanz in ihrer ursprünglichen Form nach Levenshtein (1966), sondern in einer überarbeiteten Version genutzt. 
TranskriptorInnen als auch durch den unsicheren beziehungsweise eher geringen Grad der phonologischen Exaktheit keinen geeigneten Ausgangspunkt für eine solche Berechnung. Und drittens zielt die Analyse, wie in 2.3.5 und 3.3.4 bereits angeführt, maßgeblich auf die Systemfunktion der kasustragenden Wortformen ab (vgl. Shrier 1965). Der formale Ausdruck ist, soweit er nicht die Distinktion von anderen Zellen einer paradigmatischen Reihe betrifft, kein zentraler Gegenstand der Untersuchung. Den Ausgangspunkt der Analyse stellt demnach die einzelne kasustragende Wortform unter Berücksichtigung der entsprechenden paradigmatischen Zelle dar (so beispielsweise die Form ich als Nominativform im Singular in Abgrenzung zu anderen Zellen, wie etwa der Form mich als Akkusativform im Singular). Für eine gezielte vertiefende Analyse der Typen der Kasusmarkierung z. B. zur exakten Klassifikation eines Suppletionsgrades für jeden Einzelbeleg müsste hingegen die gesamte paradigmatische Reihe (um im Beispiel zu bleiben: die vollständige paradigmatische Reihe ich/mich/mir) diesen Ausgangspunkt bilden.

Für eine solche Analyse sind bestimmte Wortarten im Korpus jedoch zu selten belegt. Aus den Ausschnitten der idiolektalen Systeme lässt sich hierfür schlichtweg keine vollständige paradigmatische Reihe rekonstruieren. Beispiele dafür sind etwa Adjektive, die zahlreiche lexikalische Varianten aufweisen, oder Possessivpronomen, die generell recht selten belegt sind. Bei der kurzen Übersicht wird daher auf eine Abstraktion der einzelnen Typen basierend auf beispielhaften paradigmatischen Reihen zurückgegriffen. Ausgehend davon werden die möglichen Suppletionsgrade der einzelnen analysierten kasustragenden Wortformen herausgearbeitet und beschrieben.

Suppletion wird dabei angelehnt an Mel'čuk (1994: 358) und Corbett (2007: 11) als Struktur intransparenter Wortformen operationalisiert, die keine Überschneidungen mit dem morphologischen Material anderer Zellen derselben paradigmatischen Reihe aufweist. Im Rahmen dieser Arbeit wird Suppletion dabei als rein synchrone Kategorie genutzt. Inwiefern zwei Wortformen, deren morphophonologische Oberfläche sich im Korpus unterscheidet, möglicherweise diachron auf dieselbe Form zurückzuführen sind, wird hier nicht berücksichtigt. Grundlage für die Klassifikation bilden ausschließlich die beispielhaft rekonstruierten paradigmatischen Reihen. Adjektive, Possessivpronomen und Indefinitpronomen ${ }^{74}$ können als die Wortarten, die - ausgehend von diesen beispielhaften Reihen - Affigierung aufweisen, betrachtet werden.

74 Hier wieder mit Ausnahme des ohnehin separat behandelten Indefinitpronomens man. 
Tab. 9: Beispielhafte paradigmatische Reihe eines Adjektivs (Sg. Mask.), rekonstruiert aus dem Transkript Unterbergen/Friedberg.

\begin{tabular}{ll}
\hline & Singular \\
\hline Nominativ & ganze \\
\hline Akkusativ & ganza \\
\hline Dativ & ganza \\
\hline
\end{tabular}

Die beispielhafte paradigmatische Reihe eines Adjektivs in Tab. 9 zeigt eine starke Transparenz zwischen dem Stamm ganz- und dem jeweiligen Flexionsaffix. Die Analyse der morphologischen Struktur der Adjektive in den Korpusdaten hat hier ein Problem mit sich gebracht, das oben bereits erwähnt wurde: Adjektive variieren verglichen mit den anderen in der Analyse untersuchten kasustragenden Wortform auf lexikalischer Ebene sehr stark. In Kombination mit der vergleichsweise niedrigen Tokenfrequenz dieser Wortart im Korpus können vollständige Paradigmen einzelner Adjektive, wie das hier angeführte, innerhalb eines Transkripts nur recht selten rekonstruiert werden. Paradigmen zur Analyse von Typen und Mustern der Kasusmarkierung müssen daher zumeist übergreifend über verschiedene Adjektive rekonstruiert werden - ein Vorgehen, das zu einer gewissen Unschärfe bei der Kategorisierung dieser Wortart führt: auf diese Weise und auf Basis des hier genutzten Korpus kann nicht überprüft werden, inwiefern etwa die lexikalisch unterschiedlichen Stämme zu verschiedenen Typen und Mustern der Kasusmarkierung neigen. Hierfür wäre eine vertiefende Analyse, die sich speziell auf Adjektive konzentriert und größere Ausschnitte idiolektaler Systeme nutzt, in denen je eine vollständige paradigmatische Reihe für jedes Adjektiv belegt ist, notwendig. Auf Basis des in Tab. 9 angeführten beispielhaften Paradigmas werden Adjektive hier zunächst verallgemeinernd einem transparenten affigierten Typus der Kasusmarkierung zugeteilt.

Tab. 10: Beispielhafte paradigmatische Reihe eines Indefinitpronomens (Sg. Mask.), rekonstruiert aus dem Transkript Öhringen_1.

\begin{tabular}{ll}
\hline & Singular \\
\hline Nominativ & oiner \\
\hline Akkusativ & oin \\
\hline Dativ & oim \\
\hline
\end{tabular}


Ähnliche Probleme wie bereits für das Adjektiv beschrieben treten, wenn auch nicht ganz so ausgeprägt, auch beim Indefinitpronomen auf. Hier ist die lexikalische Variation zwar nicht derart groß wie bei den Adjektiven. Dennoch gibt es hier auch Varianten (z. B. jedem, alles, oim), die jeweils lexikalisch bedingt verschiedene morphologische Stämme aufweisen. Mit einem Anteil von lediglich 6,2\% an der Gesamtbelegzahl sind Indefinitpronomen zudem noch niedrigfrequenter als Adjektive, was das Problem der aus Einzeltranskripten vollständig zu rekonstruierenden Paradigmen verstärkt. Tabelle 10 illustriert eine der wenigen vollständig rekonstruierbaren paradigmatischen Reihen eines Indefinitpronomens, auf dessen Basis auch diese Wortart dem Typus der transparenten Affigierung zugewiesen wird.

Tab. 11: Beispielhafte paradigmatische Reihe eines Possessivpronomens (bzw. -determinierers, 1. Ps. Sg. Mask.), rekonstruiert aus dem Transkript Königshofen/Dinkelsbühl.

\begin{tabular}{ll}
\hline & Singular \\
\hline Nominativ & mãi \\
\hline Akkusativ & $m \tilde{a} i$ \\
\hline Dativ & $m a \tilde{i n}$ \\
\hline
\end{tabular}

Diesem Typus lassen sich zudem auch die Possessivpronomen zuteilen. Tabelle 11 illustriert ein entsprechendes Paradigma.

Anders als Adjektive und Indefinitpronomen weisen Possessivpronomen keine große lexikalische Variation auf (sie variieren lediglich zwischen morphologisch ähnlich strukturierten Formen wie beispielsweise sãi, mãi oder dei). Die Abstraktion basiert auch hier auf den wenigen Beispielen, die sich aus der noch deutlich geringeren Datenmenge von nur 2,3\% aller Korpusbelege ableiten lassen doch gelingt diese Abstraktion bedeutend einfacher. Wie in Tab. 11 illustriert, lässt sich auch hier ein Stamm relativ transparent von einem Affix separieren, das zumindest einen Kasus distinkt markiert.

Ähnlich, wenn auch hier nicht ganz unstrittig, verhält es sich beim Demonstrativpronomen. Die verhältnismäßig große Belegmenge der Demonstrativpronomen (13,3\%) vereinfacht hier die Rekonstruktion beispielhafter paradigmatischer Reihen, wie etwa Tab. 12. 
Tab. 12: Beispielhafte paradigmatische Reihe eines Demonstrativpronomens, rekonstruiert aus dem Transkript Königshofen/Dinkelsbühl.

\begin{tabular}{ll}
\hline & Singular \\
\hline Nominativ & där \\
\hline Akkusativ & den \\
\hline Dativ & dem \\
\hline
\end{tabular}

Anhand dieses beispielhaften Paradigmas ist man versucht, das Demonstrativpronomen ebenso wie etwa das Adjektiv oder das Possessivpronomen dem Typus der transparenten Affigierung zuzuordnen. Beim Demonstrativpronomens besteht der Stamm jedoch lediglich aus einem einzelnen Segment /d/. Das von Corbett (2007: 15-16) angeführte Kriterium der Transparenz von Stamm und Affix ist durch das geringe morphophonologische Material des Stammes deutlich weniger stark ausgeprägt als bei dem oben beschriebenen Typus. Hier wird daher ein eigener Typus der Kasusmarkierung angesetzt. Dieser weist zwar ebenfalls grundlegend Affigierung auf, ist jedoch auf morphologischer Ebene etwas intransparenter als der oben erwähnte.

Äußerst komplex gestaltet sich schließlich die Klassifikation bei Artikeln. Sowohl Definit- als auch Indefinitartikel können teilweise, wenn es sich beispielweise um Vollformen handelt, demselben Typus wie das Demonstrativpronomen zugeteilt werden (vgl. Tab. 13 und 14). Auch sie zeigen hier insofern eine Form der Affigierung, als zumindest ein Einzellaut in jeder Zelle des Paradigmas erhalten bleibt und durch ein Affix ergänzt wird.

Tab. 13: Beispielhafte paradigmatische Reihe eines Definitartikels (Sg. Mask.), rekonstruiert aus dem Transkript Bieringen/Künzelsau.

\begin{tabular}{ll}
\hline & Singular \\
\hline Nominativ & $d^{\prime} r$ \\
\hline Akkusativ & $d a$ \\
\hline Dativ & dem \\
\hline
\end{tabular}


Tab. 14: Beispielhafte paradigmatische Reihe eines Indefinitartikels (Sg. Mask.), rekonstruiert aus dem Transkript Deilingen/Tuttlingen.

\begin{tabular}{ll}
\hline & Singular \\
\hline Nominativ & an \\
\hline Akkusativ & $a n$ \\
\hline Dativ & $a m$ \\
\hline
\end{tabular}

Vorrangig bedingt durch die Tendenz zur Klitisierung bzw. Formenreduktion, die sich häufig bei Artikeln und Pronomen findet, können jedoch nicht alle Belege der Definit- und Indefinitartikel diesem Typus der Kasusmarkierung zugeteilt werden. Beispielhaft illustrieren Tab. 15 und 16 Artikelparadigmen, die formale Reduktion aufweisen. Sie zeigen verschiedene Aspekte auf, die eine Zuordnung zum oben genannten Typus der Affigierung eines kurzen Stammes bzw. Einzellauts ausschließen. Zunächst kann bei den reduzierten bzw. klitischen Formen nicht zwischen Stamm und Affigierung unterschieden werden - je nach Kliseform wäre hier lediglich ein (verkürzter) Stamm oder ein Teil des Affixes vorhanden. Sie sind also stark intransparent.

Tab. 15: Beispielhafte paradigmatische Reihe eines Definitartikels (Sg. Mask.) mit reduzierten Formen, rekonstruiert aus dem Transkript Bieringen/Künzelsau.

\begin{tabular}{ll}
\hline & Singular \\
\hline Nominativ & $d^{\prime} r$ \\
\hline Akkusativ & $d e$ \\
\hline Dativ & $m$ \\
\hline
\end{tabular}

Tab. 16: Beispielhafte paradigmatische Reihe eines Indefinitartikels (Sg. Mask.) mit reduzierten Formen, rekonstruiert aus dem Transkript Deilingen/Tuttlingen.

\begin{tabular}{ll}
\hline & Singular \\
\hline Nominativ & $a$ \\
\hline Akkusativ & $n$ \\
\hline Dativ & $m a$ \\
\hline
\end{tabular}


Wie sich aus Tab. 16 ablesen lässt, können sich die reduzierten Formen innerhalb eines Paradigmas zudem in ihrer morphophonologischen Oberfläche derart unterschieden, dass totale Suppletion auftritt. Tabelle 15 hingegen zeigt ein Paradigma, in dem verschieden stark reduzierte Formen in den einzelnen Zellen auftreten. Dort kann eher partielle Suppletion angesetzt werden (da in diesem Fall zumindest in zwei Zellen eine partielle Übereinstimmung vorhanden ist). Dabei ist jedoch zu berücksichtigen, dass sich die Klassifikation der reduzierten Formen als suppletiv hier ausschließlich auf die aus dem Korpus rekonstruierten paradigmatischen Reihen und damit auf die in den Transkripten notierte morphophonologische Oberfläche stützt. Inwiefern diese suppletive Struktur jeweils im gesamten Idiolekt oder gar im gesamten Diasystem bei reduzierten Formen auftritt, lässt sich aus diesen Daten nicht erschließen.

Auch Nübling (1992: 223) beschreibt die morphologische Struktur dialektaler Klitika anhand von Daten des Berndeutschen als nicht segmentierbare und eher zu Suppletion neigende Portmanteaustrukturen. Ein gewisser Zusammenhang zwischen Formenreduktion und Suppletion und ein damit verbundener Unterschied in der morphologischen Struktur von vollen und reduzierten Formen wäre also kein unrealistisches Szenario.

Zudem wird häufig ein funktionaler Unterschied zwischen Reduktions- und Vollformen beschrieben. Dazu werden - angelehnt an eine Tradition aus Orts- und Dialektgrammatiken - zwei getrennte Paradigmen für Artikel (und teilweise auch für Personalpronomen) angesetzt: eines für volle und eines für reduzierte Formen (vgl. dazu beispielsweise Studler 2013), denen dann wiederum verschiedene Funktionen zugeteilt werden (vgl. beispielsweise diskurspragmatische Funktionen bei Dirani 2020, Weiß/Dirani 2019 oder der Ausdruck von semantischer und pragmatischer Definitheit bei Nübling 2005 und Himmelmann 1997). Angelehnt an die Argumentation von Howe (1996) lässt sich dieser funktionale Unterschied nun auch bezüglich der Kasusdistinktion vermuten: Es ist zu überprüfen, ob klitische Artikelformen einen größeren Anteil distinkter Belege zeigen als Vollformen.

Dabei stellt eine Operationalisierung von Klise, die alle klitischen Formen im Korpus erfasst, ein großes methodisches Problem dar: Grundsätzlich sollten dadurch alle reduzierten Artikelformen erfasst werden, die keine vollkommen eigenständigen Wortformen mehr darstellen, d.h. sowohl einfache als auch spezielle Klitika (vgl. Terminologie in Nübling 1992). Die prosodisch-phonetischen Merkmale, die für eine umfassende und exakte Definition der Klitika angesetzt werden können, lassen sich aus den Korpusdaten jedoch nicht problemlos erkennen. Nach Nübling (1992: 12-19) müssten etwa die Tilgung der Junktur (und damit die Anlehnung der Artikelform an ein anderes Wort), ein innerer Sandhi (und damit der Verlust von phonologischem Material gegenüber einer entsprechenden Vollform) 
sowie Akzentlosigkeit (und damit die prosodischen Eigenschaften der Form) zur Abgrenzung von vollen Artikelformen nachgewiesen werden.

Die Transkriptionskonvention in Ruoff (1984) sieht die Kennzeichnung einer engen Verbindung zwischen zwei Wortformen (durch einen Bindestrich) vor (vgl. Ruoff 1984: XVI); eine Tilgung der Junktur zu kennzeichnen wäre dort demnach grundsätzlich möglich. Das entsprechende Zeichen wird jedoch von den TranskriptorInnen äußerst selten und ausschließlich für die Allegroform ham-mer und nicht bei (möglichen) klitischen Verbindungen genutzt. Auch die in 3.4 .1 beschriebenen Apostrophe, die zur Markierung dialektnormabweichender Synkopen und Apokopen genutzt werden sollten (vgl. Ruoff/Gabriel 1998: 28 und Ruoff 1984: XVI), können nicht zur eindeutigen Klassifikation von klitischen oder reduzierten Artikelformen genutzt werden. Zwar könnten sie durchaus als Markierung eines internen Sandhi interpretiert werden, da sie jedoch erstens auch außerhalb von Kontexten mit (möglicherweise) klitischen Artikeln auftreten (z. B. m'r) und zweitens lediglich in Fällen genutzt werden, die als von der „Dialektnorm“ abweichend klassifiziert wurden, muss von einem eher uneinheitlichen Gebrauch dieser Zeichen ausgegangen werden. Die phonologisch oder gar phonetisch wenig exakte Transkription in Ruoff (1984) ermöglicht daher keine Klassifikation der Artikelform nach diesen beiden Merkmalen.

Generell stellt eine exakte Aufteilung der Belege in verschiedene Reduktionsstufen bei gesprochensprachlichen und damit zu einem breiten Spektrum an Variation neigenden Daten eine gewisse theoretische und besonders auch methodische Hürde dar. Halpern (2001: 119), der verschiedene Modelle zur Definition und Abgrenzung (verschiedener) Reduktionsformen und Klisestufen bündelt, sieht die Grundlage einer theoretischen Operationalisierung von Klitika in der exakten Abgrenzung verschiedener Reduktionsformen voneinander sowie von entsprechenden Vollformen: „We must ask whether there is a significant split between simple and special clitics, between verbal clitiics and (other) special clitics, between clitics and affixes, or between clitics and independent words“ (Halpern 2001: 119). Für die eindeutige Definition einer Wortform als (einfaches oder spezielles) Klitikon ist folglich deren exakte Abgrenzung zu anderen reduzierten Formen sowie zur Vollform notwendig. Was Halpern (2001) hier aus theoretischer Perspektive fordert, ist jedoch in den hier genutzten Daten methodisch nicht umsetzbar. Die exakte Abgrenzung dieser Formen sowie die dazu notwendige Definition der entsprechenden Grenzwerte in gesprochensprachlichen Daten bedürfen einer ausführlichen phonetischen, prosodischen und darauf aufbauenden morphosyntaktischen Analyse, die auf Grundlage der Transkripte in Ruoff (1984) nicht geleistet werden kann.

Eine eindeutige Unterteilung der Belege in volle, reduzierte und klitische Paradigmen kann folglich nicht vorgenommen werden. Zumindest ein kleiner Einblick in den Zusammenhang von Klise und Kasusdistinktion ist jedoch möglich. 
Zwar können die einzelnen Reduktionsgrade auf Basis der Transkripte nicht zuverlässig abgebildet werden, doch lassen sich zumindest solche Formen untersuchen, die einer Minimalhypothese von Klitika entsprechen. Für die Artikelformen, die in der Transkription lediglich durch ein einziges konsonantisches Zeichen (z. B. $d, m$, $n$ ) wiedergegeben sind, kann ein eigener Akzent auch auf Grundlage der Transkription ausgeschlossen werden. Sie können damit eindeutig als Klitika identifiziert werden, während alle übrigen Formen (z. B. d'r, da, em) zwar durchaus ebenfalls reduzierte Formen bzw. Klitika darstellen können, dieser Minimalhypothese aber nicht entsprechen und hier daher nicht als Klitika gewertet werden. Bei diesen als nicht eindeutig klitisch klassifizierten Formen handelt es sich mit großer Wahrscheinlichkeit nicht ausschließlich um Vollformen; auch teilweise reduzierte Formen, die aber aufgrund der Transkription nicht eindeutig als solche abzugrenzen sind, werden zu dieser Kategorie gezählt.

Ein $\chi^{2}$-Test zeigt, dass die der Minimalhypothese entsprechenden klitischen Artikelformen (Definitartikel: 2.339 klitische Belege, 36\%, Indefinitartikel: 64 klitische Belege, 3,8\%) hier signifikant häufiger mit Distinktion assoziiert sind als die als nicht eindeutig klitisch klassifizierten Formen. ${ }^{75}$ Ein Zusammenhang zwischen reduzierten Formen im Artikelparadigma und Kasusdistinktion deutet sich damit bereits in dieser stark vereinfachten Operationalisierung an und sollte in vertiefenden Arbeiten (auf Grundlage von entsprechend geeigneten Daten) näher untersucht werden.

In Bezug auf die hier besprochene Fragestellung lässt sich also zunächst grundsätzlich festhalten, dass es sich bei beiden Artikeln - sowohl bei definiten als auch bei indefiniten - um Wortarten handelt, denen sich kein einheitlicher Typus der Kasusmarkierung zuordnen lässt. Vielmehr scheinen diese Wortarten ein recht breites Spektrum verschiedener Typen der Affigierung von Einzellauten bis hin zur totalen Suppletion abzudecken, wobei hier insbesondere reduzierte bzw. klitische Artikelformen für die suppletiveren Strukturen und vermutlich auch für einen Anteil distinkter Belege verantwortlich sind.

Etwas eindeutiger als die Typen der Artikel lassen sich die der Personalpronomen bestimmen. Diese variieren je nach grammatischer Kategorie, wie etwa Person, Numerus und Genus, aber teilweise auch bedingt durch Formenreduktion ähnlich dem Artikel - zwischen den Typen der partiellen und totalen Suppletion

75 Ausgewertet wurde eine einfache Kreuztabelle mit den jeweils binären Variablen, Kasusdistinktion (SYNKRETISCH/DISTINKT) und Klise (KLITISCH/NICHT-KLITISCH), mit einem Pearsons' $\chi^{2}$ Test ( Chi = 38,617, df =1, p -Wert = 5,157e-10). Die Residualwerte zeigen eine positive Assoziation von klitischen und distinkten $(3,1)$ sowie von nicht-klitischen und synkretischen Belegen $(2,4)$ und eine negative Assoziation von klitischen und synkretischen $(-3,7)$ sowie nichtklitischen und distinkten $(-2,5)$ Belegen. 
(vgl. dazu auch Howe 1996: 49-50 zu morphologischen Strukturen germanischer Personalpronomen).

So lässt sich in beispielhaften Paradigmen wie in Tab. 17 angelehnt an Corbett (2007: 15-16) die Überschneidung der morphophonologischen Oberfläche zwischen den einzelnen Zellen zur Skalierung heranziehen. Bei Dativ und Akkusativ kann man so etwa einen aus einem Einzellaut bestehenden Stamm ansetzen, der durch das entsprechende Affix modifiziert wird, wohingegen hier im Nominativ dann ein alternativer Stamm angesetzt werden muss und damit partielle Suppletion vorliegt. Ein ähnliches, allerdings standarddeutsches Beispiel führt auch Weber (1987: 311) an und klassifiziert es recht vorsichtig als „leichte Suppletion?“ - eine terminologische Unsicherheit, die mit Corbetts (2007) feinerer Ausdifferenzierung vermieden werden kann. In Beispielen wie etwa beim Paradigma des Personalpronomens im Maskulinum Singular in Tab. 18 kann man hingegen von totaler Suppletion ausgehen - hier gibt es keine Übereinstimmung der morphophonologischen Oberfläche. ${ }^{76}$

Tab. 17: Beispielhafte paradigmatische Reihe eines Personalpronomens (1. Ps. Sg.), rekonstruiert aus dem Transkript Hirrlingen/Tübingen.

\begin{tabular}{ll}
\hline & Singular \\
\hline Nominativ & $i$ \\
\hline Akkusativ & $m i$ \\
\hline Dativ & $m r$ \\
\hline
\end{tabular}

Tab. 18: Beispielhafte paradigmatische Reihe eines Personalpronomens (3. Ps. Sg. Mask.), rekonstruiert aus dem Transkript Frankenbach/Heilbronn.

\begin{tabular}{ll}
\hline & Singular \\
\hline Nominativ & $\ddot{a r}$ \\
\hline Akkusativ & $n$ \\
\hline Dativ & $m$ \\
\hline
\end{tabular}

76 Inwiefern auch hier, ähnlich wie beim Artikel, eine Art der Formenreduktion strukturell mit bestimmten Typen der Kasusmarkierung interagiert, lässt sich auf Basis der Daten kaum feststellen. Aufgrund der etwas höheren lexikalischen Variation von Personalpronomen (beispielsweise $i, e r, s e$ ) wäre hierfür eine deutlich komplexere Operationalisierung von Klitika und unter Umständen auch größere phonologische Exaktheit der Transkription notwendig. 
Abbildung 18 illustriert zusammenfassend die hier angeführte abstrahierte Klassifikation der einzelnen Wortarten nach den angesetzten Typen der Kasusmarkierung:

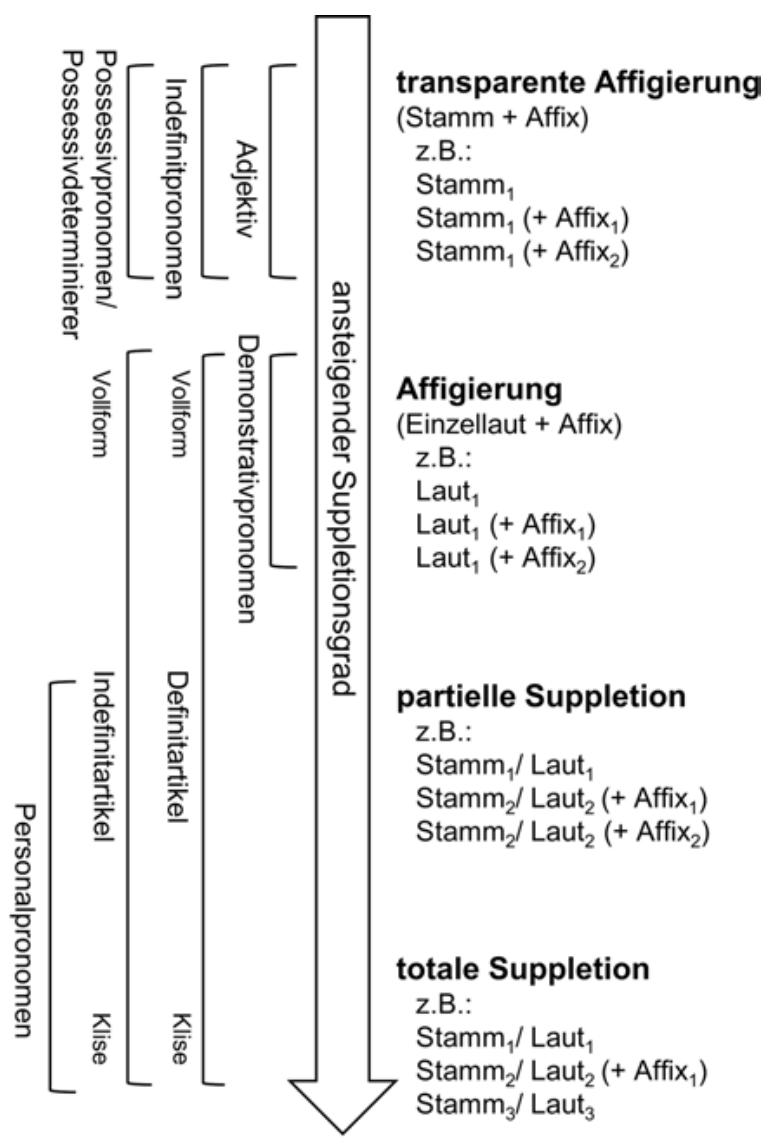

Abb. 18: Angenommenes Spektrum von Typen der Kasusmarkierung für die untersuchten kasustragenden Wortarten; aufgeführt nach Suppletionsgrad.

Abbildung 18 führt die grundlegenden Typen der Kasusmarkierung auf, die im Spektrum zwischen den Polen der Affigierung und der totalen Suppletion angesiedelt sind, und stellt damit eine erste grobe Anpassung der nach Dammel (2008) für die Verbalflexion angesetzten Suppletionsgrade an die morphologische Struktur der kasustragenden Wortarten dar. Im Bereich der eher affigierten Strukturen wurde hier nicht der von Dammel (2008: 26) für verbale Kategorien erarbeitete Typus der Stammalternation übernommen. Dafür wurde ein spezieller, weniger transparenter Typus der Affigierung eingesetzt. Die einzelnen Wortarten 
sind mit ihrem jeweiligen Typenspektrum verzeichnet, wodurch sich eine Staffelung der einzelnen Wortarten nach den Suppletionsgraden zeigt. Die Annahme, dass reduzierte Formen bzw. Klitika bei Artikeln für die suppletivsten Typen der Kasusmarkierung verantwortlich sind, wurde auch in die Abbildung integriert.

Die Wortarten werden nun mit ihrem jeweiligen Anteil an synkretischen und distinkten Formen im Korpus abgebildet, wodurch sich erste Rückschlüsse auf deren Bedeutung für die Kasusdistinktion im Diasystem ableiten lassen. Abbildung 19 zeigt, dass der Definitartikel (52,9\%) und das Personalpronomen $(63,5 \%)$ am häufigsten durch distinkte Formen belegt sind. Dieses Ergebnis basiert auf der Gesamtmenge aller Belege für die einzelnen kasustragenden Wortarten - unabhängig von anderen morphologischen Kategorien, wie Numerus, Genus und Person, die ebenfalls Einfluss auf die Kasusdistinktion üben können (vgl. 4.2.3) und deren Frequenz sich möglicherweise zwischen den einzelnen Wortarten im Korpus deutlich unterscheidet.

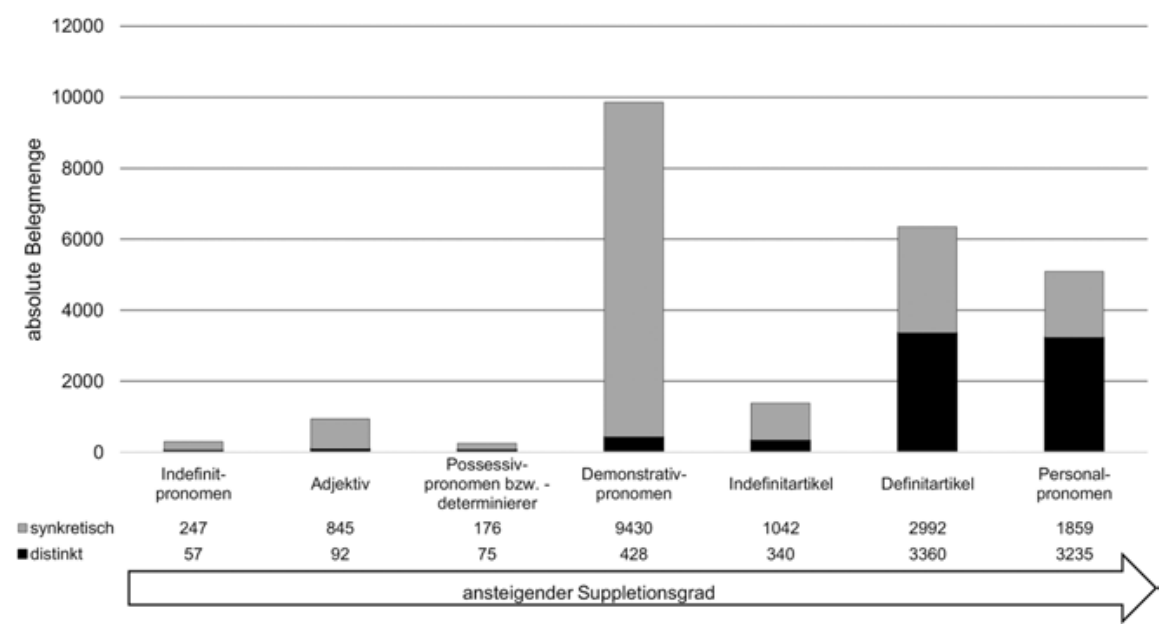

Abb. 19: Anteil synkretischer und distinkter Belege der einzelnen Wortformen; grob sortiert nach deren angenommenem Suppletionsgrad.

Die Wortarten, deren Spektrum eher affigierte Typen enthält, scheinen hier für einen Großteil der synkretischen Belege im Korpus verantwortlich zu sein - ein Zusammenhang, der aufgrund der relativ niedrigen Belegzahlen von Possessivpronomen, Adjektiven und Indefinitpronomen und den damit verbundenen Problemen vorsichtig formuliert ist und sich daher hauptsächlich auf die Erkenntnisse zum Demonstrativpronomen stützt. Auch für den Indefinitartikel liegen verglichen mit den beiden anderen zur Suppletion neigenden Wortarten wenig Belege vor, wodurch er sich schlecht in diese Kategorisierung einfügen lässt. 
Es lässt sich dennoch erkennen, dass tatsächlich zwei der Wortarten, deren Typenspektrum partielle bzw. teilwiese auch totale Suppletion enthält - der Definitartikel und das Personalpronomen - einen Großteil der distinkten Belege ausmachen. Ein kausaler Zusammenhang lässt sich hier zwar aufgrund der nicht formabbildenden Konzeption der Analyse, die bereits zu Beginn dieses Kapitels angesprochen wurde, nicht nachweisen. Dennoch weisen die Daten deutlich darauf hin, dass es tatsächlich eben diese eher suppletiven Typen sind, die die Hauptlast der distinkten Kasusmarkierung im untersuchten Diasystem tragen. Die zentrale Rolle der Suppletion, die sich bereits bei Howe (1996: 70) mit Blick auf die Personalpronomen angedeutet hat, konnte grundlegend auch unter Berücksichtigung eines breiteren Spektrums kasustragender Wortarten bestätigt werden: Die Kategorie Kasus wird im untersuchten Diasystem folglich weniger durch Affigierung als vielmehr durch Suppletion ausgedrückt.

Diese Erkenntnis stützt das grundlegend wortbasierte Vorgehen der Analyse (vgl. 3.3.1). Da die Formen, die den distinkten Ausdruck der Kategorie Kasus tragen, eher schwer segmentierbar und suppletiv sind, eignet sich die vollständige kasustragende Wortform besser als Ausgangspunkt für eine Analyse der Kasusmarkierung als das kasusmarkierende Morphem. Eine zusätzliche morphembasierte Analyse ${ }^{77}$ ist nur dann von Vorteil, wenn auch die Wortarten wie beispielsweise Adjektiv, Possessivpronomen und Indefinitpronomen, die zwar Kasus tragen, ihn aber nicht unbedingt distinkt markieren, untersucht werden sollen.

\subsubsection{Muster der Kasusmarkierung: Interdependenz grammatischer Kategorien}

Die vorangegangenen Kapitel haben bereits die für das Diasystem anzusetzenden Kasus Nominativ, Akkusativ und Dativ definiert sowie erste Erkenntnisse zu den morphologischen Strukturen geliefert, die zur Distinktion dieser Kasus beitragen. Die Argumentation wurde dabei an einigen Stellen schon mit ersten Analysen zur Assoziation der kanonischen Kasus, der Wortarten und darin vertretenen morphologischen Strukturen mit Kasussynkretismus und -distinktion untermauert. Während sich 4.2.2 jedoch noch stark auf die Formseite der Kasusmarkierung konzentriert hat, widmet sich dieses Kapitel nun der Systemfunktion. Es bietet eine vertiefende Darstellung der Gliederung von synkretischen und distinkten Belegen im Korpus und damit den Mustern der Kasusmarkierung.

77 Auch in der hier vorgestellten Analyse wurde in bestimmten Fällen, z. B. bei den Adjektiven, teilweise auf eine morphembasierte Analyse zur Klassifikation von Synkretismus und Distinktion zurückgegriffen. 
Dabei wird die Distinktionshierarchie der angesetzten abstrakten Kasus noch einmal aufgegriffen und empirisch basiert abgebildet, welche Kasus frequent zum Formenzusammenfall neigen und in welchen Kontexten synkretische beziehungsweise distinkte Markierung der einzelnen Kasus auftritt. Die Abbildung eben dieser Kontexte und der dort gefundenen Muster der Kasusmarkierung bietet aber an erster Stelle die Möglichkeit, Interdependenzen zwischen der Kategorie Kasus und anderen (morphologischen) grammatischen Kategorien, namentlich Person, Numerus und Genus, aufzuzeigen. Es wird dargestellt, inwiefern diese Kategorien bestimmte Muster der Kasusmarkierung präferieren, welche dieser Kategorien besonders starken Einfluss auf die Kasusdistinktion üben und wo mögliche Ursachen für diese Präferenzen liegen.

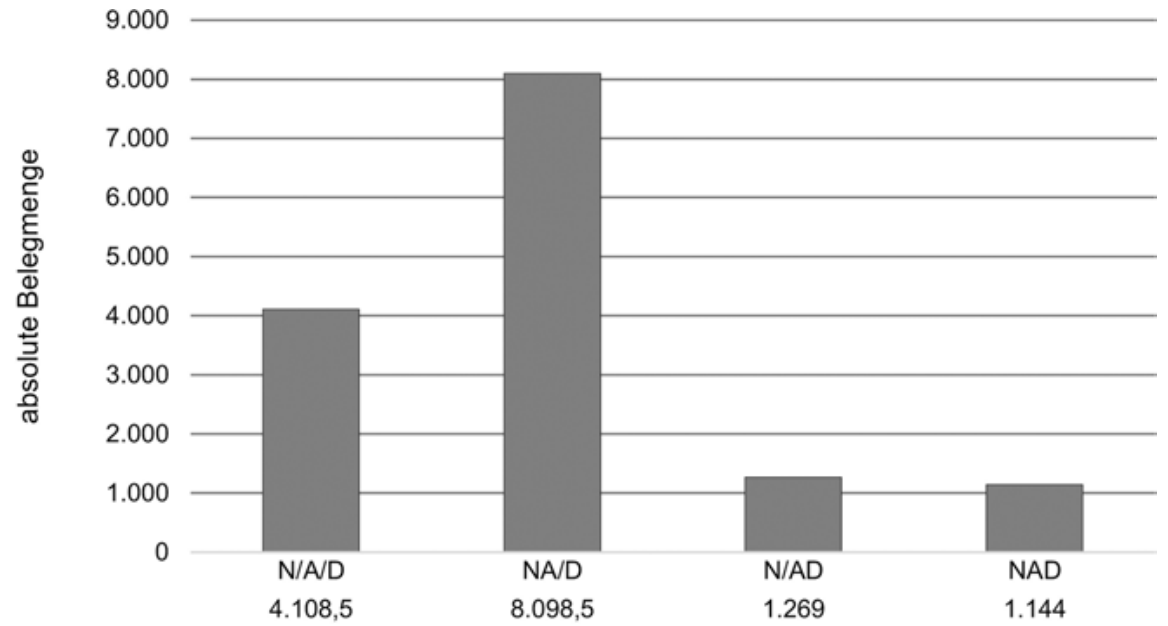

Abb. 20: Anteile der Muster der Kasusmarkierung im gesamten Korpus.

Abbildung 20 gibt einen Überblick über die quantitativen Anteile der Muster der Kasusmarkierung, die im Korpus auf Basis der in 3.5.4 vorgestellten automatisierten Auswertung der Datenbank ermittelt wurden. ${ }^{78}$ Grundlage dafür waren 14.620 Belege. ${ }^{79}$

78 Wie bereits in 3.5.4 ausführlich dargestellt, wurde hier nicht jeder Beleg in der Datenbank einem Muster zugeteilt. Lediglich die Belege, für die eine vollständige paradigmatische Reihe im entsprechenden Transkript belegt war, wurden in diese Übersicht sowie in die folgenden Detaildarstellungen integriert.

79 Den übrigen Belegen (5.178) konnte entweder keine der beiden Variablen zur Kasusdistinktion zugeordnet werden oder sie wurden auf Basis einer unvollständigen paradigmatischen 
Aus der Übersicht ist zunächst zu erkennen, dass es sich bei dem synkretischen Muster NA/D um das mit Abstand frequenteste Muster der Kasusmarkierung handelt, das einen Anteil von 55,4\% der gesamten den Mustern zugrundeliegenden Daten einnimmt, gefolgt von dem vollständig distinkten Muster N/A/D mit einem Anteil von 28\%. Deutlich geringer sind hingegen die Anteile des synkretischen Musters N/AD (8,7\%) und des totalen Synkretismus NAD (7,8\%). Die im kanonischen Paradigma angesetzte volle Distinktion aller drei Kasus wird damit lediglich bei einem Anteil von 28\% der untersuchten kasustragenden Wortformen erreicht. Dennoch lässt sich in 92,2\% Prozent der Belege ein Muster nachweisen, das mindestens einen Kasus distinkt markiert (N/A/D, N/AD und NA/D). Das bedeutet, dass es sich bei den zahlreichen verschiedenen in der Analyse untersuchten Wortformen tatsächlich zu einem Großteil um Kasusmarker, wenn auch nicht unbedingt um kanonische Kasusmarker (nach der Definition in 3.3.5), handelt. Lediglich das Muster des totalen Synkretismus weist keinerlei Markierung eines Kasus auf; die Wortformen, die dieses Muster tragen, können damit nicht als Kasusmarker klassifiziert werden (vgl. dazu 3.3.5).

Die in Abb. 20 aufgeführten Muster lassen sich nach Person, Numerus und Genus übersichtlich gliedern. Die Gliederung spiegelt dabei die Abhängigkeit der Kasusmarkierung von eben diesen grammatischen Kategorien wider und bietet damit einen Einblick in die Auswirkungen der Interdependenz grammatischer Kategorien im Kasusdiasystem. Dependenz wird hier mit Aikhenvald/Dixon (1998: 56) als Abhängigkeitsverhältnis definiert, bei dem die Ausprägung der einen Kategorie (z. B. der Anzahl der Kasusdistinktionen) von der jeweiligen Ausprägung der anderen Kategorie (z. B. des jeweiligen Numerus) abhängig ist. Basierend auf dieser Definition lassen sich die hier untersuchten grammatischen Kategorien im deutschen System in der folgenden inkrementellen Hierarchie abbilden:

\section{Person $>$ Numerus $>$ Genus $>$ Kasus}

Person wurde hier oberhalb des Numerus angesetzt, da die Numerusdistinktion an einer einzigen (zugegebenermaßen recht kleinen) Stelle im System von der Personendistinktion abhängt: bei der höflichen Anrede der 2. Person gibt es keine formale Numerusdistinktion (Sg. Sie, Pl. Sie) ${ }^{80}$ Genus wird an dritter Stelle aufgeführt, da Genusdistinktion nur in der 3. Person und auch dort ausschließlich im Singular auftritt. Genus ist damit sowohl von Person als auch von Nume-

Reihe als SYNKRETISCH klassifiziert und damit nicht in die Übersicht der Muster, die ja auf vollständigen paradigmatischen Reihen basiert, aufgenommen.

80 Für eine diachrone und strukturelle Erklärung dieser Sonderstellung der höflichen Anrede im Pronominalsystem siehe Simon (2003). 
rus abhängig. Die Distinktion der Kasus ist nun wiederum von allen dreien Kategorien abhängig - ein Umstand, den Aikhenvald/Dixon (1998: 74) auch auf Basis typologischer Daten, also sprachsystemübergreifend, feststellen:

If there should be any dependency between case and another system, we would expect case to be dependent on the other system.

(Aikhenvald/Dixon 1998: 74)

Erklärt wird die niedrige Position von Kasus in der Hierarchie durch seine Funktion im grammatischen System. Aikhenvald/Dixon (1998: 73-74) gehen vom Satz als Grundeinheit des grammatischen Systems aus. Je stärker eine grammatische Kategorie den Satz beeinflusst, desto höher steht sie auf der Dependenzhierarchie. So stehen etwa die verbalen Kategorien (z. B. Tempus, Aspekt und Evidenzialität) höher in der Hierarchie, da sie das Prädikat des Satzes beeinflussen, während die übrigen, nominalen Kategorien (z. B. Person, Numerus, Genus) nur die vom Prädikat geforderten Argumente semantisch spezifizieren. Kasus hingegen drückt lediglich die syntaktische Funktion dieser Argumente in der Oberflächenstruktur aus und übt damit einen eher geringen Einfluss auf den Satz selbst aus (vgl. Aikhenvald/Dixon 1998: 74-75). Die Ausprägungen der Kategorie Kasus sind also von der jeweiligen Ausprägung der anderen grammatischen Kategorien abhängig.

Dabei ist zu erwarten, dass sich die allgemeine Interdependenzhierarchie (10) auch in der Abhängigkeit der Kasusdistinktion von den einzelnen anderen grammatischen Kategorien spiegelt, es sich bei diesen Kategorien folglich nicht um unabhängige Variablen handelt. Genus wird beispielsweise nur im Singular differenziert. Die Genusdifferenzierung kann sich folglich nur im Singular auf die Kasusdistinktion auswirken. Zudem sind die einzelnen grammatischen Kategorien nicht homogen über die verschiedenen kasustragenden Wortarten verteilt. So werden die 1. und 2. Person ausschließlich am Personalpronomen markiert, während die 3. Person ein deutlich größeres Wortartenspektrum aufweist.

Eine Operationalisierung der Abhängigkeitsverhältnisse der Kasusdistinktion in den Korpusdaten muss diese Interdependenzen der anderen grammatischen Kategorien berücksichtigen. Hierzu wird nun die bereits in 3.3.4 angeführte Distinktionsquote genutzt. Damit können die einzelnen Belege im Korpus und die ihnen zugeordneten Werte von Kasussynkretismus und -distinktion direkt in Bezug zu den einzelnen grammatischen Kategorien gesetzt werden. Sie gibt den Anteil an distinkten Belegen relativ zur Gesamtbelegmenge der entsprechenden grammatischen Kategorie wieder. Über den Mittelwert der einzelnen Distinktionsquoten können Unterschiede der verschiedenen Wortarten und Kontexte, die für die einzelnen Kategorien relevant sind, ausdifferenziert werden.

Erklärungsansätze für die jeweiligen Distinktionsquoten bieten Übersichten zu den Mustern der Kasusmarkierung im Korpus aufgeteilt nach den einzelnen grammatischen Kategorien (vgl. Tab. 20, 21 und 22). Sie zeigen, welche 
abstrakten Kasus in der entsprechenden Kategorie als distinkt und welche als synkretisch klassifiziert worden sind, d. h. welche Systemfunktionen hinter den Distinktionsquoten stehen.

Tabelle 19 zeigt die Distinktionsquoten aufgeteilt nach den Werten der Kategorie Numerus. Für die jeweiligen Quoten wurde ein Mittelwert aus den Wortarten berechnet, in denen eine Numerusdistinktion möglich ist: Personalpronomen bei der 1. Person und Personalpronomen, Definit- und Indefinitartikel (nur im Singular), Demonstrativpronomen, teilweise Indefinitpronomen und Adjektive. ${ }^{81}$ Die Belege für die 2. Person (352 Belege) reichen für zuverlässige Aussagen nicht aus und werden daher im Folgenden nicht berücksichtigt. ${ }^{82}$

Tab. 19: Distinktionsquoten der Belege kategorisiert nach Numerus.

\begin{tabular}{|c|c|c|c|}
\hline & & Singular & Plural \\
\hline 1. & Person & $99 \%$ & $59 \%$ \\
\hline 3. & Person & $33 \%$ & $6 \%$ \\
\hline \multicolumn{2}{|c|}{ gesamt (Mittelwert) } & $66 \%$ & $33 \%$ \\
\hline & $\begin{array}{l}\text { h Numerus } \\
\text { rminierte Differenz }\end{array}$ & & $33 \%$ \\
\hline
\end{tabular}

Aus der Tabelle ist zu erkennen, dass sich die gemittelten Distinktionsquoten von Singular und Plural um 33\% unterscheiden. Diese Differenz kann als vom Numerus determiniert gewertet werden. Im Singular tritt deutlich mehr Kasusdistinktion auf als im Plural. Die Quoten unterscheiden sich zudem in den verschiedenen Personen. Die 1. Person zeigt im Singular wie im Plural eine deutlich höhere Distinktionsquote als die 3. Person.

Die Differenz in der Distinktionsquote, die durch den Numerus determiniert ist, spiegelt sich auch in den Mustern der Kasusmarkierung. Die Domäne der vollen Distinktion (des Musters N/A/D) ist in jedem Fall der Singular. Im Plural treten ausschließlich verschiedene synkretische Muster auf, d.h. solche, die

81 Es wurden lediglich die Indefinitpronomen berücksichtigt, die sich bezüglich Numerus, Genus und Person klassifizieren lassen (z. B. einer). Possessivpronomen und -determinierer wurden hier nicht berücksichtigt, da sie nicht für jeden Wert der untersuchten Kategorien im Korpus auftreten.

82 Da es sich bei den Daten im Korpus zum Großteil um initiierte Erzählmonologe handelt, ist die Anrede einer 2. Person grundsätzlich relativ selten und zudem stark durch die höfliche Anrede geprägt, da in den meisten Fällen der Explorator angesprochen wird. 
zwischen mindestens zwei Zellen Synkretismus aufweisen. Tabelle 20 gibt einen Überblick über die Muster der Kasusmarkierung im Plural.

Tab. 20: Anteile verschiedener Muster der Kasusmarkierung im Plural.

\begin{tabular}{lrrrrrr}
\hline Person & \multicolumn{2}{c}{ NA/D } & \multicolumn{2}{c}{ N/AD } & \multicolumn{2}{c}{ NAD } \\
\hline 1. & 0 & $0 \%$ & 890 & $25,9 \%$ & 0 & $0 \%$ \\
\hline 3. & 1.771 & $51,5 \%$ & 0 & $0 \%$ & 781 & $22,7 \%$ \\
\hline & $\mathrm{n}=3.442$ & & & & & \\
\hline
\end{tabular}

Die Tabelle zeigt die Anteile der verschiedenen Muster der Kasusmarkierung, die im Korpus im Plural auftreten. Über die Hälfte der Belege zeigt das Muster NA/D, in dem der Dativ distinkt markiert wird, während Nominativ und Akkusativ synkretisch sind. Anders ist dies beim Muster N/AD, wo Nominativ distinkt markiert wird. Auch Tab. 20 zeigt eine deutliche Differenzierung der Muster nach der Kategorie Person: Während das Muster NA/D in der 3. Person auftritt, sind alle 890 Belege für das Muster N/AD für Personalpronomen der 1. Person Plural belegt eine Kategorie, die wiederum ausschließlich dieses Muster zeigt. Mit diesem Muster lässt sich auch die deutlich höhere Distinktionsquote der Pluralbelege der 1 . Person gegenüber denen der 3. Person in Tab. 19 erklären: Wie in Tab. 7 aufgeführt, ist der Nominativ im Korpus mit 59,9\% deutlich häufiger belegt als der Akkusativ (23,5\%) und der Dativ (16,7\%). Ein Muster, das eine Nominativdistinktion enthält, führt damit zu einer deutlich größeren Menge an distinkten Belegen als eines, das einen distinkten Dativ aufweist. Zudem tritt ausschließlich in der 3. Person ein moderater Anteil des totalen Synkretismus mit dem Muster NAD auf, was zu zusätzlichen synkretischen Belegen führt. Die Muster im Plural lassen sich damit vollständig anhand der Kategorie Person gliedern, die des Singulars weisen hingegen deutlich größere Mustervariation auf, was die Tab. 21 illustriert.

Das Muster der vollen Distinktion N/A/D findet sich, wie bereits erwähnt, ausschließlich im Singular. Auch dort ist das Muster jedoch auf maskuline Kategorien der 3. Person sowie auf die 1 . Person beschränkt, ${ }^{83}$ während das Femininum und Neutrum der 3. Person ausschließlich synkretische Muster aufweisen. Hierbei handelt es sich nahezu ausschließlich um das Muster NA/D, das, wie bereits oben erwähnt, verglichen etwa mit dem Muster N/AD relativ wenige distinkte Formen erwarten lässt. Die höhere Distinktionsquote im Singular stützt

83 Wie bereits erwähnt, reichen die Daten, die zur 2. Person vorhanden sind, nicht für eine automatisierte Auswertung der Muster der Kasusmarkierung aus. 
Tab. 21: Anteile verschiedener Muster der Kasusmarkierung im Singular.

\begin{tabular}{|c|c|c|c|c|c|c|c|c|c|}
\hline \multirow{2}{*}{$\frac{\text { Person }}{1 .}$} & \multirow[t]{2}{*}{ Genus } & \multicolumn{2}{|c|}{ N/A/D } & \multicolumn{2}{|c|}{ NA/D } & \multicolumn{2}{|c|}{ N/AD } & \multicolumn{2}{|c|}{ NAD } \\
\hline & & 1.727 & $94,8 \%$ & 0 & $0 \%$ & 94 & $5,2 \%$ & 0 & $0 \%$ \\
\hline & & $\mathrm{n}=1.821$ & & & & & & & \\
\hline \multirow[t]{6}{*}{3.} & Maskulinum & $2.381,5$ & $70,8 \%$ & 636,5 & $18,9 \%$ & 238 & $7,1 \%$ & 106,5 & $3,2 \%$ \\
\hline & & $\mathrm{n}=3.362$ & & & & & & & \\
\hline & Femininum & 0 & $0 \%$ & 1.940 & $91,8 \%$ & 2 & $0,09 \%$ & 172 & $8,1 \%$ \\
\hline & & $n=2.114$ & & & & & & & \\
\hline & Neutrum & 0 & $0 \%$ & 3.748 & $97,8 \%$ & 0 & $0 \%$ & 84 & $2,2 \%$ \\
\hline & & $\mathrm{n}=3.832$ & & & & & & & \\
\hline
\end{tabular}

sich damit auf die Belege der 1. Person und des Maskulinums, die offenbar eine Sonderstellung im Kasusdiasystem einnehmen.

Abbildung 21 zeigt nun auch den Einfluss der Kategorie Genus. Letzterer spiegelt sich grundsätzlich in unterschiedlichen Mustern der Kasusmarkierung beim Maskulinum verglichen mit dem Femininum und Neutrum, was in Abb. 21 illustriert ist.

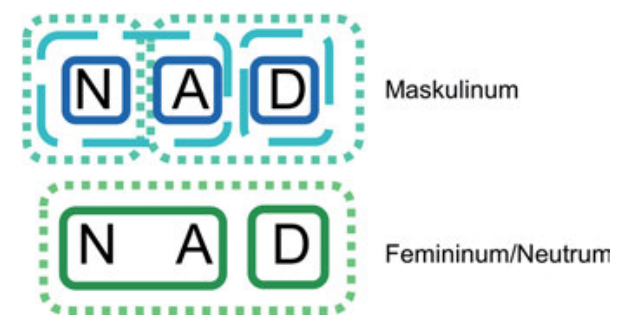

Abb. 21: Genusspezifische Differenzen bei den Mustern der Kasusmarkierung.

Die durchgezogenen Linien zeigen in Abb. 21 die prominenten Muster der Kasusmarkierung an. Die durchbrochenen Linien illustrieren Muster, die auf wenige Kategorien beziehungsweise Kontexte beschränkt sind. Femininum und Neutrum zeigen eine starke Tendenz zum Muster NA/D. Die zwei Belege für N/AD im Femininum können hier als Artefakt der Zuordnung von Synkretismus und Distinktion beziehungsweise der Transkriptionskonvention gewertet werden. Zwar tritt in beiden Kategorien auch der totale Synkretismus (NAD) auf, dieser scheint jedoch eine eher untergeordnete Rolle zu spielen. Auch im Maskulinum finden sich zwar Belege für das im Femininum und Neutrum dominante Muster NA/D 
sowie für die beiden anderen synkretischen Muster. Das dominante Muster, das beim größten Anteil an Belegen auftritt, ist hier jedoch die vollständige Distinktion N/A/D. Auffällig ist hier zunächst, dass das Maskulinum im Vergleich zu den beiden anderen Genera eine deutlich größere Mustervariation aufweist, was auch Abb. 21 reflektiert. Dies ist ein Aspekt, der womöglich durch die diachrone Entwicklung der Kasusmarkierung in den einzelnen Genera zu erklären ist: Während das Muster NA/D im Neutrum seit Beginn der deutschen Sprachgeschichte und im Femininum schon seit dem Mittelhochdeutschen belegt ist, wurde die Distinktion N/A/D beim Maskulinum über einen langen Zeitraum erhalten. Nivellierungstendenzen treten hier vermutlich erst seit dem Frühneuhochdeutschen auf (vgl. dazu Häckel/Walch 1988: 184 und 191) und haben bis heute nur einen Teil des Untersuchungsraums ergriffen: Die verschiedenen Muster im Maskulinum sind an bestimmte geographische Räume gebunden (vgl. dazu näher 4.3), wodurch sich die verhältnismäßig starke Mustervariation gegenüber den beiden anderen Genera begründen lässt.

Insbesondere der große Anteil des Musters N/A/D im Maskulinum führt zu einer deutlich höheren Distinktionsquote im Vergleich zu Femininum und Neutrum, was in Tab. 22 aufgegliedert ist. Die Distinktionsquoten der Genera unterscheiden sich um durchschnittlich 58,5\%. Damit differiert die Distinktionsquote bei Genusunterschieden fast doppelt so stark wie bei Numerusunterschieden, wo diese Differenz 33\% ausmacht (vgl. Tab. 21).

Tab. 22: Distinktionsquoten der Belege im Singular der 3. Person kategorisiert nach Genus.

\begin{tabular}{lccr}
\hline & Maskulinum & Femininum & Neutrum \\
\hline gesamt (Mittelwert) & $72 \%$ & $16 \%$ & $11 \%$ \\
\hline Mittelwert Maskulinum-Femininum & $56 \%$ & \\
\hline Mittelwert Maskulinum-Neutrum & & $61 \%$ \\
\hline durch Genus determinierte Differenz & & $58,5 \%$ \\
\hline
\end{tabular}

Die Ursache für den auffällig starken bzw. diachron langen Erhalt der Kasusdistinktion und die damit verbundene Sonderstellung des Maskulinums lässt sich in den semantischen Eigenschaften der jeweiligen Referenten vermuten, die bereits in 2.2.2.5 wurde unter Bezug auf Bank (2007), Krifka (2009) und Alber/Rabanus (2011) als mögliche Einflussfaktoren auf die Kasusdistinktion angeführt wurden: Unter der Annahme, dass unbelebte Entitäten ohnehin stärker mit patientivischen Rollen assoziiert sind, gehen diese Arbeiten davon aus, dass Kategorien, die eher 
unbelebte Referenten aufweisen, stärker zu Synkretismus neigen. Als Grund dafür wird die distinkte Zuordnung semantischer Rollen aufgeführt, die hier bereits durch das Merkmal der Belebtheit auf semantischer Ebene gewährleistet ist. Anhand der Korpusdaten kann dieser Annahme nun ausgehend von der semantischen Annotation der Einzelbelege empirisch erstmals auch auf Grundlage der Tokenfrequenz nachgegangen werden. Abbildung 22 illustriert die anteilige Belegmenge der einzelnen Genera im Korpus aufgeteilt nach den Stufen der Belebtheitshierarchie.

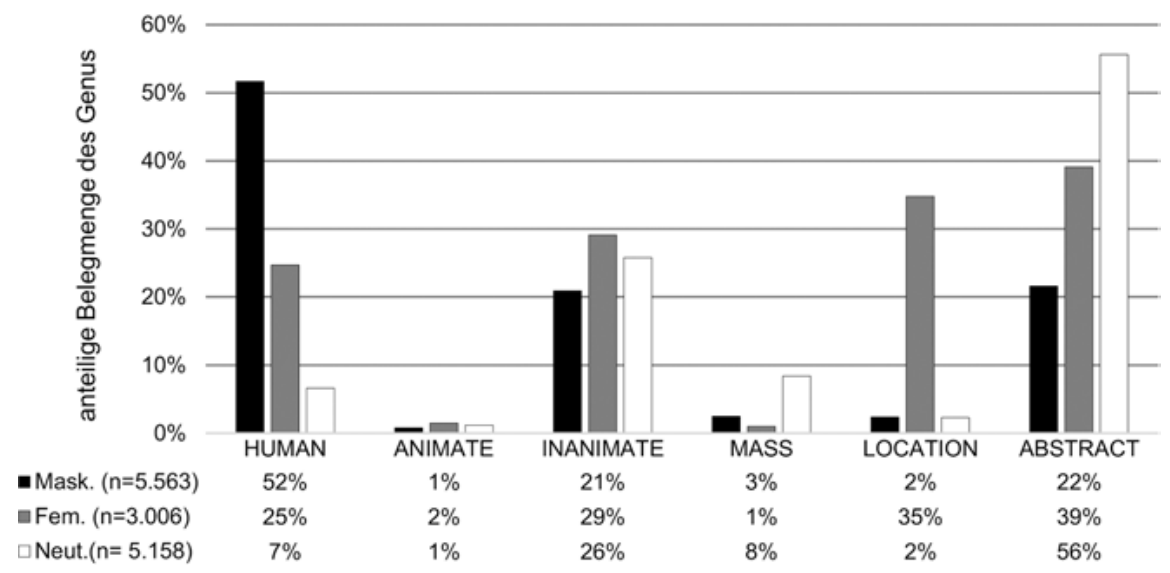

Abb. 22: Anteil der Genus an Belebtheitsstufen in den Korpusdaten.

Hier lässt sich erkennen, dass das Maskulinum bei den menschlichen Referenten (HUMAN) dominiert, während die anderen beiden Genera auf den unteren Belebtheitsstufen dominant vertreten sind. ${ }^{84}$ Damit bestätigen die Daten die Annahme, die Krifka (2009: 155-158) auf Grundlage der Auswertung einer Type-Liste von Nomen aus Ruoff (1981) trifft. Er geht davon aus, dass die niedrige Zahl belebter Referenten (neben weiteren Faktoren) im Femininum als Ursache für die Ausbildung des Nominativ-Akkusativ-Synkretismus zu werten ist: „Perhaps feminine NPs in German simply did not refer often enough to animate beings to create sufficient functional pressure to keep up the N/A-distinction“ (Krifka 2009: 155). Die Korpusdaten zeigen nun erstens, dass sich nicht nur die Typen-, sondern auch die Tokenfrequenz der unterschiedlich belebten Referenten in den kasustragen-

84 Ein entsprechender $\chi 2$-Test zeigt mit einem $\mathrm{p}$-Wert von $2.2 \mathrm{e}-16(\mathrm{Chi}=3031.3, \mathrm{df}=10)$ eine starke Assoziation. Zwar zeigt sich hier mit Cramer'sV = 0,334 lediglich eine mittlere Effektstärke, ein grundlegender Zusammenhang zwischen den Kategorien ist jedoch auch hier zu erkennen. 
den Wortarten der einzelnen Genera unterscheidet, und zweitens, dass dieser Unterschied nicht nur die Distinktion ANIMATE vs. INANIMATE, sondern im Speziellen die Distinktion HUMAN vs. - HUMAN betrifft.

Eine Erklärung für die starke Tendenz zur Nominativdistinktion beim Maskulinum (im Gegensatz zum vollständigen Nominativ-Akkusativ-Synkretismus bei Femininum und Neutrum) könnte also in der Dominanz menschlicher Referenten liegen, deren distinkte Nominativmarkierung im Sprachsystem relevanter zu sein scheint als die bei weniger belebten Referenten. Diese Beobachtung wird durch die Erkenntnisse zur diachronen Ausbreitung des Musters NA/D gestützt: Die Reihenfolge der Kategorien, in denen Nominativ und Akkusativ nivelliert wurden (Neutrum > Femininum > Maskulinum), spiegelt sich im jeweiligen Anteil menschlicher Belege im Korpus, was auf einen gewissen Zusammenhang zwischen diachronem Erhalt der Kasusdistinktion und dem Anteil an menschlichen Referenten in einer grammatischen Kategorie hinweist.

Auf den Einfluss, den die Kategorie Person auf die Muster der Kasusmarkierung übt, wurde bereits bei der Ausführung zum Numerus verwiesen. Wie bereits im Plural weichen auch im Singular die Muster der 1. Person deutlich von denen der 3. Person ab: Zwar dominiert auch hier - ähnlich oder eher noch ausgeprägter als im Maskulinum - das Muster der vollständigen Distinktion N/A/D (94,4\%). Wie aber auch im Plural neigt die 1. Person in den seltenen Fällen von Synkretismus zum Muster N/AD (5,2\%). Die 1. Person in Singular und Plural zeigt also ganz grundsätzlich andere Mustern der Kasusmarkierung als die 3. Person, wo N/AD nur vereinzelt Maskulinum belegt ist. Ebenso wie das Maskulinum nimmt damit auch die 1. Person eine Sonderrolle im Kasusdiasystem ein. Sie weist im Singular einen deutlich höheren Anteil an vollständiger Distinktion auf und neigt bei Synkretismus zu Mustern des Akkusativ-Dativ-Synkretismus anstatt zu denen des Nominativ-Akkusativ-Synkretismus, was wiederum, wie oben bereits angeführt, zu einer höheren Distinktionsquote beiträgt.

Im Diasystem deutet sich damit der Ausbau einer personeninduzierten Spaltung in den Mustern der Kasusmarkierung an. Personeninduzierte Spaltungen („Splits“) innerhalb von Kasussystemen werden in typologischen Arbeiten häufig beschrieben (vgl. dazu insbesondere Siewierska 2004: 63-66). Prominentestes Beispiel ist der Ergativ-Split (Silverstein 1976, Comrie 1981), der zumindest teilweise personeninduziert ist. Neben Belebtheit löst dort auch das Auftreten eines Pronomens der 1. oder 2. Person den Wechsel zwischen von Ergativ-Absolutiv- zu einem Nominativ-Akkusativ-System aus (vgl. Silverstein 1976 zum Kasussystem des Dyirbal). Von einer solch fundamentalen Spaltung ist das untersuchte Diasystem zwar deutlich entfernt, allerdings kann hier durchaus die Tendenz einer grundlegenden Differenzierung anhand der Personen beobachtet werden: Während in der 1. Person immer eine Nominativdistinktion gewährleistet ist und 
dort eher der ansonsten im Diasystem so distinkte Dativ zu Synkretismus neigt, ist die Nominativdistinktion bei der 3. Person nur in bestimmten maskulinen Kontexten gewährleistet, während der Dativ immer distinkt markiert wird. Abbildung 23 illustriert diese Tendenz einer personeninduzierten Spaltung in den Mustern der Kasusmarkierung und deutet dabei die Richtung dieses Ausbaus an.

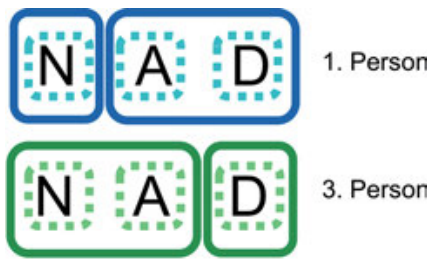

Abb. 23: Personenspezifische Differenzen in den Mustern der Kasusmarkierung.

Die durchgezogenen Linien zeigen die dominanten synkretischen Muster an, während die unterbrochenen Linien die beiden distinkten Muster, die jeweils auf bestimmte Kategorien (auf den Singular bzw. das Maskulinum) reduziert sind, illustrieren. Dass eben diese Kategorien ein gewisses Variationsspektrum aufweisen und die distinkten Muster, zumindest im Maskulinum, diachron erste Nivellierungstendenzen zeigen, verstärkt den Eindruck, der in Abb. 23 angedeutet ist. Für ein vollständiges Bild der personeninduzierten Muster der Kasusmarkierung wären an dieser Stelle jedoch Informationen zu den Mustern der 2. Person notwendig.

Tab. 23: Distinktionsquoten der Belege aller Wortarten kategorisiert nach Person.

\begin{tabular}{lrr}
\hline & 1. Person & 3. Person \\
\hline Gesamt (Mittelwert) & $82 \%$ & $20 \%$ \\
\hline durch Person determinierte Differenz & & $62 \%$ \\
\hline
\end{tabular}

Die unterschiedlichen Muster der 1. und 3. Person führen wortartenübergreifend zu einem relativ großen Unterschied der gemittelten Distinktionsquoten dieser beiden Werte. Tabelle 23 führt die Distinktionsquote der 1. im Vergleich zu der der 3. Person auf. Die Tabelle zeigt eine Differenz von 62\% zwischen den beiden Werten. Person zeigt damit einen höheren Einfluss auf die Kasusdistinktion als die beiden übrigen grammatischen Kategorien.

Verglichen mit den Differenzwerten der beiden anderen grammatischen Kategorien ist der direkte Einfluss der Kategorie Person insbesondere auch aufgrund der Spaltung der Muster der Kasusmarkierung, die sie auslöst, als äußerst stark einzuschätzen. Dies kann auf verschiedene Aspekte zurückgeführt werden: 
Zunächst ist ein gewisser Einfluss der unterschiedlichen Wortartenspektren zu berücksichtigen: Die 1. Person wird ausschließlich durch Personalpronomen ausgedrückt, während diese bei der 3. Person nur einen gewissen Anteil (lediglich $16,5 \%$ aller Belege der 3. Person im Korpus) des Wortartenspektrums ausmacht. Der Anteil an stärker suppletiven Strukturen ist folglich bei der 1. Person deutlich größer als bei der 3. Person. Die hohe Distinktionsquote der 1. Person spiegelt damit zu einem gewissen Teil auch die Erkenntnisse zu den Typen der Kasusmarkierung (4.2.2) wider. Hinter der Quotendifferenz jedoch ausschließlich einen Effekt der Unterscheidung zwischen Personalpronomen und anderen, weniger suppletiven Wortarten anzunehmen, würde allerdings zu kurz greifen: Tab. 24 zeigt die Distinktionsquoten der 1. und 3. Person, die ausschließlich auf Belegen von Personalpronomen beruhen. Auch hier zeigt sich eine deutliche Differenz der Distinktionsquoten. Diese ist mit lediglich (46\%) allerdings geringer als die, die auf Basis des wortartenübergreifenden Vergleichs ermittelt wurde.

Tab. 24: Distinktionsquoten der Belege der Personalpronomen kategorisiert nach Person.

\begin{tabular}{lrr}
\hline & 1. Person & 3. Person \\
\hline Personalpronomen (Mittelwert) & $82 \%$ & $36 \%$ \\
\hline durch Person determinierte Differenz & & $46 \%$ \\
\hline
\end{tabular}

Eine deutlich nachhaltigere Erklärung für dieses Phänomen lässt sich auf semantischer Ebene ansetzen: Während die 1. Person durchweg eine hohe Stufe der Belebtheitshierarchie einnimmt (SELF), kann die 3. Person alle übrigen Stufen (d. h. Human (4.290 Belege, 30,9\%), Animate (204 Belege, 1,5\%), InANimate (3.586 Belege, 25,8\%), MASs (550 Belege, 4\%), LoCATION (384 Belege, 2,8\%) und ABSTRACT (4.894 Belege, 35,2\%)) einnehmen. Damit bildet sie ein deutlich größeres Spektrum der Belebtheitsstufen ab, das zudem die höchste Stufe (SELF) nicht erreicht. Die Referenten der 3. Person sind folglich grundsätzlich weniger belebt als die der 1. Person. Dieser Unterschied geht mit einem Unterschied in den semantischen Rollen, die die Referenten jeweils einnehmen, einher. Abbildung 24 gibt einen Überblick über die Proto-Rollen, die im Korpus jeweils von den Belegen der 1. und der 3. Person ausgedrückt werden.

Während die Belege der 1. Person überwiegend agentivischere Rollen (РROTOAgENS und PROTO-REZIPIENT) einnehmen, dominieren in der 3. Person die weniger agentivischen Rollen (Proto-Patiens und Proto-Location). ${ }^{85}$ Diese Differen-

85 Ein entsprechender $\chi 2$-Test zeigt mit einem $p$-Wert von 2.2e-16 $(\mathrm{Chi}=2617.5, \mathrm{df}=3)$ eine starke Assoziation und mit Cramer’s V =0,362 eine mittlere Effektstärke. 


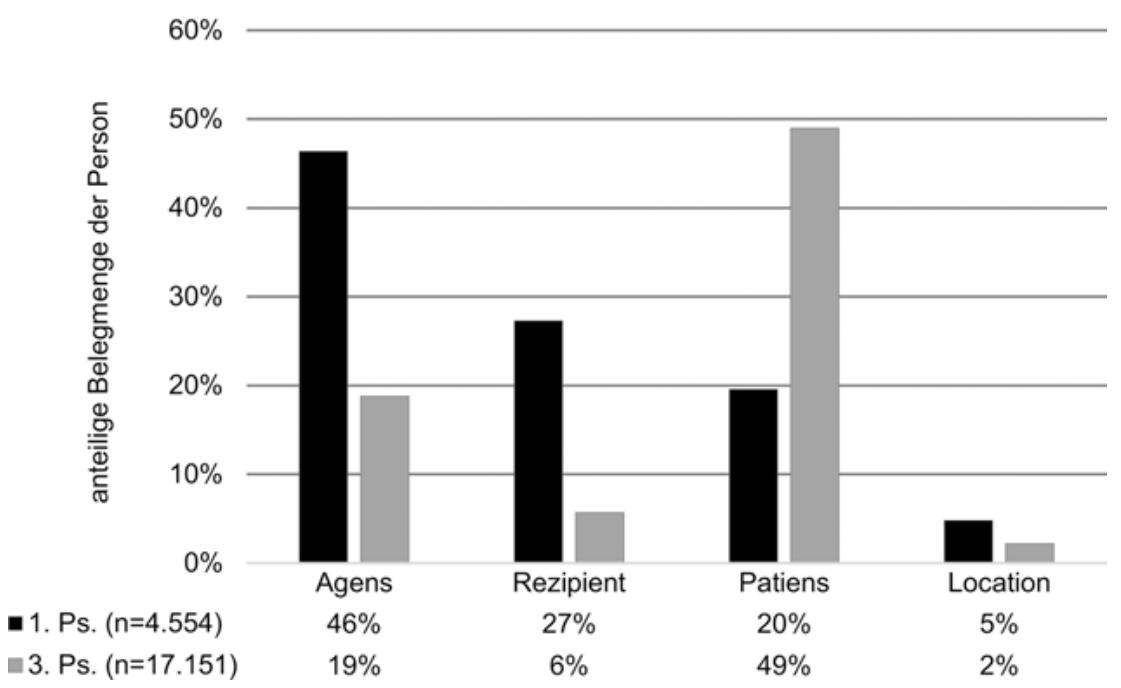

Abb. 24: Anteil der Personen an den Proto-Rollen in den Korpusdaten.

zen zwischen der 1. und 3. Person weisen auf eine semantische Ursache der personeninduzierten Spaltung in den Mustern der Kasusmarkierung und die damit einhergehende große Differenz der Distinktionsquote hin, wie sie sich ähnlich bereits für die genusspezifischen Unterschiede abgezeichnet hat. In den grammatischen Kategorien, die häufig agentivische Rollen und höhere Belebtheitsstufen einnehmen, scheint die Kasusdistinktion generell, besonders aber die Nominativdistinktion (z. B. N/A/D bzw. N/AD) stärker erhalten zu sein als in den Kategorien, die weniger agentivische Rollen und niedrigere Belebtheitsstufen einnehmen. Diese neigen bei erhaltener Kasusdistinktion eher zur Unterscheidung von Akkusativ und Dativ (z. B. NA/D) und damit zu einer Differenzierung verschiedener patientivischer Rollen.

Bezogen auf die Dependenz der Kategorie Kasus im Diasystem lassen sich diese grammatischen Kategorien auf Grundlage der Distinktionsquoten abschließend wie folgt hierarchisieren:

\section{(11) Person $>$ Genus $>$ Numerus}

Sowohl die Übersicht über die Muster der Kasusmarkierung als auch die Distinktionsquoten haben gezeigt, dass Kasusdistinktion im Diasystem hauptsächlich durch die 1. Person (82\%) und durch die 3. Person Singular Maskulinum (72\%) 
getragen wird. Als mögliche Ursache dafür wurde deren Assoziation mit stark agentivischen bzw. menschlichen Referenten angeführt. Pluralische, feminine und neutrale Wortformen der 3. Person Singular zeigen deutlich niedrigere Distinktionsquoten und spielen damit eine eher untergeordnete Rolle bei der Kasusmarkierung im untersuchten Diasystem.

\subsubsection{Zentrale und periphere Kasusmarker}

Im vorigen Teilkapitel wurde auf Basis der wortartenübergreifenden Erkenntnisse ein erster Zusammenhang zwischen Kasus und den Kategorien Genus, Numerus und Person aufgezeigt. In diesem Teilkapitel werden nun die kasustragenden Wortarten in den verschiedenen grammatischen Kategorien einzeln auf ihre Rolle im Kasusdiasystem hin analysiert. Ziel ist es, herauszuarbeiten, welche der kasustragenden Wortformen grundsätzlich Kasusmarker sind und welche darüber hinaus dem Ideal der kanonischen Kasusmarkierung (vgl. 3.3.5) besonders nahe kommen und damit zentral zur Kasusdistinktion im Diasystem beitragen.

In 3.3.5 wurden die Kriterien für die kanonische Kasusmarkierung definiert. Diese bestehen in einer vollständigen paradigmatischen Reihe und einer distinkten Wortform in jeder Zelle dieser Reihe. Letzteres lässt sich in der Analyse auf verschiedene Arten quantifizieren: Vollständige Kasusdistinktion äußert sich im Muster N/A/D. Die Wortarten, die dieses Muster aufweisen - das Personalpronomen der 1. Person Singular sowie verschiedene Wortarten in der 3. Person Singular Maskulinum - kommen damit grundlegend auch als zentrale Kasusmarker in Frage. Eben diese Wortarten zeigen im Diasystem jedoch eine gewisse Mustervariation. Wenn auch zu geringeren Anteilen sind hier neben N/A/D auch synkretische Muster belegt (vgl. 4.2.3). Das kanonische Ideal eines Kasusmarkers wird also nicht vollständig erreicht.

Um alle Wortarten anhand dieses Kriteriums in ihrer Nähe zur kanonischen Kasusmarkierung zu staffeln, ist eine feinkörnigere Ausdifferenzierung notwendig, die durch die Distinktionsquote erzielt werden kann. Ein kanonischer Kasusmarker müsste nach dieser Definition eine Distinktionsquote von 100\% aufweisen. Die exakte Quote zeigt, welche kasustragenden Wortformen grundsätzlich Kasusmarkierungen sind, d. h. welche mindestens einen distinkten Beleg aufweisen, und wie diese anhand des Anteils distinkter Belege hierarchisiert werden können, $d . h$. welche eine zentrale und welche eine eher periphere Rolle bei der Kasusmarkierung im Diasystem spielen. Tabelle 25 gibt einen Überblick über die analysierten kasustragenden Wortformen, ihre Belegmenge im Korpus sowie ihre jeweilige Distinktionsquote. 
Tab. 25: Kasustragende Wortarten sortiert nach Distinktionsquote im Korpus.

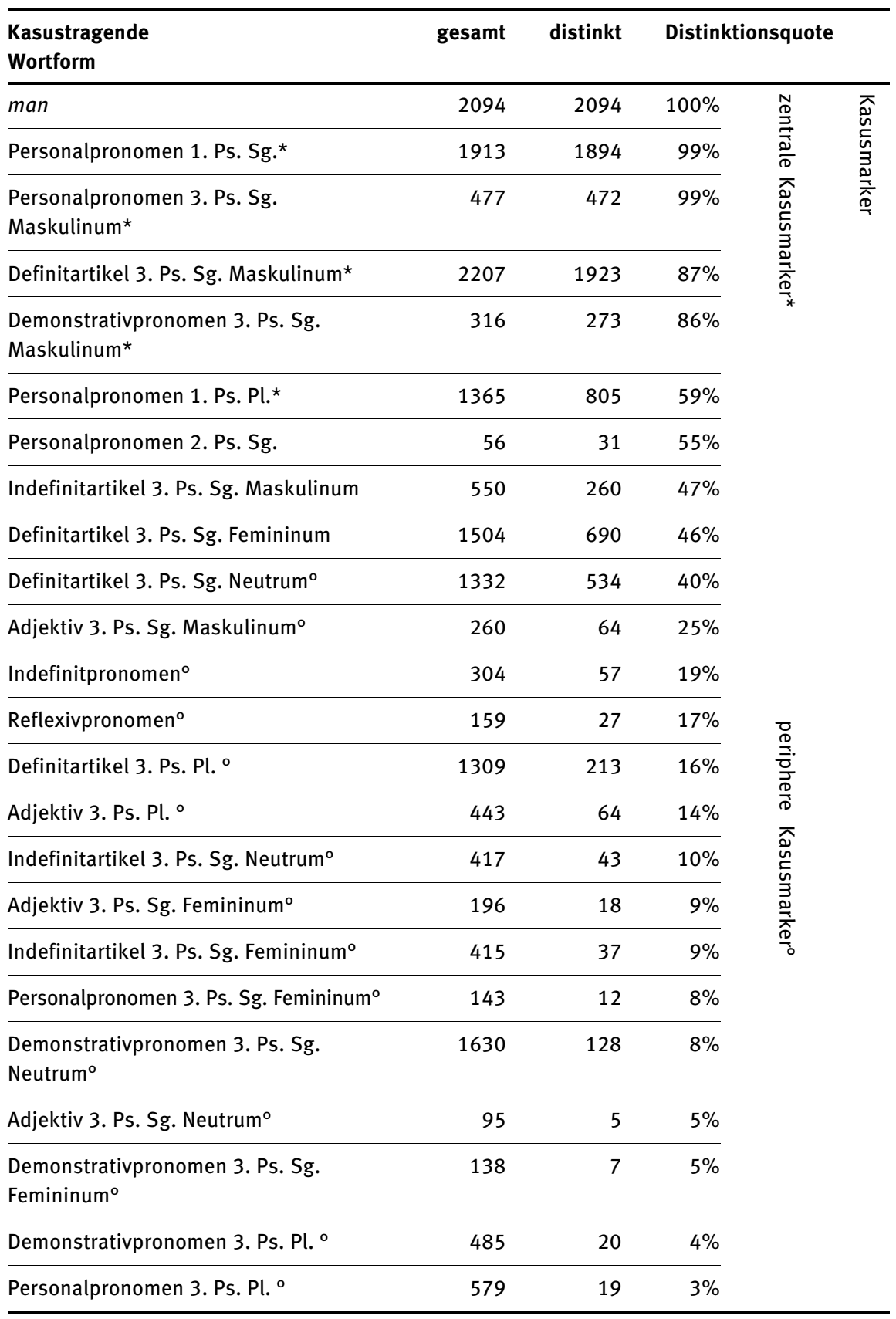


Tab. 25 (fortgesetzt)

\begin{tabular}{|c|c|c|c|}
\hline $\begin{array}{l}\text { Kasustragende } \\
\text { Wortform }\end{array}$ & gesamt & distinkt & Distinktionsquote \\
\hline $\begin{array}{l}\text { Possessivpronomen/-determinierer } \\
\text { 3. Ps. Sg. Mask. }{ }^{\circ}\end{array}$ & 0 & 0 & $0 \%$ \\
\hline $\begin{array}{l}\text { Possessivpronomen/ -determinierer } \\
\text { 3. Ps. Sg. Fem. }\end{array}$ & 0 & 0 & \\
\hline $\begin{array}{l}\text { Possessivpronomen/-determinierer } \\
\text { 3. Ps. Sg. Neut. }\end{array}$ & 0 & 0 & \\
\hline Possessivpronomen 3. Ps. Pl. ${ }^{\circ}$ & 0 & 0 & \\
\hline Personalpronomen 3. Ps. Sg. Neutrumº & 929 & 0 & \\
\hline Personalpronomen 2. Ps. Pl. ${ }^{\circ}$ & 6 & 0 & \\
\hline
\end{tabular}

Die Tabelle führt die kasustragenden Wortformen gegliedert nach ihrer Distinktionsquote im Korpus auf. Die Gesamtzahl der Wortformen, auf der die Distinktionsquote basiert, umfasst dabei nur die Belege, die eindeutig als synkretisch oder distinkt klassifiziert werden konnten. Die Belege, die nach dem in 3.5.3 beschriebenen Vorgehen keinem dieser beiden Werte zugeteilt werden konnten, zählen nicht zur Gesamtzahl der Belege.

Ganz grundsätzlich lässt sich in der Tabelle erkennen, dass das Personalpronomen der 3. Person Singular Neutrum, das der 2. Person Plural ${ }^{86}$ sowie die Possessivpronomen keine Kasusmarkierungen darstellen, da sie eine Distinktionsquote von $0 \%$ aufweisen. Insbesondere bezüglich des Personalpronomens der 3. Person Singular Neutrum scheint dies zunächst verwunderlich zu sein: Hier wäre zumindest eine Dativdistinktion (z. B. es/es/ihm) zu erwarten gewesen. Im Korpus findet sich jedoch keine vollständige paradigmatische Reihe; es gibt keinen Beleg für eine Form dieses Personalpronomens, die den abstrakten Dativ ausdrückt. Damit ist hier auch das Kriterium der vollständigen paradigmatischen Reihe nicht erfüllt.

86 Auf das Problem der geringen Belegmenge zur 2. Person in den Korpusdaten wurde bereits mehrfach verwiesen. Erkenntnisse zur 2. Person in diesem Kapitel sind daher wohl nicht auf andere Datentypen (z. B. Gesprächsdaten) generalisierbar. 
Dieses Beispiel illustriert die grundlegende Eigenschaft der hier angeführten Distinktionsquote: Sie spiegelt - anders als etwa die Synkretismusquote bei Alber/Rabanus (2011) - nicht die im Diasystem grundsätzlich angelegten, sondern die tatsächlich im Korpus belegten Muster der Kasusmarkierung wider. Damit erfasst die Quote lediglich die Kasusdistinktionen, die so frequent auftreten, dass sie im Korpus nachzuweisen sind. Die Distinktionsquote, die hier für die kasustragenden Wortformen ermittelt wurde, ist damit auch in gewissem Maß von dem Ausschnitt aus den idiolektalen Systemen abhängig, der im Korpus enthalten ist. In die Quote werden nur die Belege aufgenommen, die eindeutig als distinkt klassifiziert werden konnten. Wie bereits in 3.5.3 ausgeführt, erlaubt die Analysemethode jedoch eine einfachere Klassifikation von synkretischen als von distinkten Belegen, da für erstere unter Umständen zwei paradigmatische Zellen ausreichen, während für letztere eine vollständige paradigmatische Reihe notwendig ist. Es ist folglich anzunehmen, dass es im Diasystem tatsächlich einen deutlich größeren Anteil an distinkten Belegen gibt, als die hier angeführten Distinktionsquoten vermuten lassen. Die Distinktionsquote stellt damit eine Art Minimalhypothese der Kasusmarkierung dar.

Das Kriterium der vollständigen paradigmatischen Reihe ist auch beim Indefinitpronomen man sowie beim Reflexivpronomen nicht erfüllt. Die Belege des Reflexivpronomens wurden dann als distinkt klassifiziert, wenn die beiden vorhandenen Zellen distinkte Formen aufgewiesen haben, d. h. wenn keine Distinktion von Akkusativ und Dativ besteht. man tritt, wie bereits ausgeführt, ausschließlich im Nominativ, das Reflexivpronomen hingegen nur im Akkusativ und Dativ auf. Die Belege von man wurden immer als distinkt klassifiziert. Sie stellen jedoch keinen kanonischen Kasusmarker dar, obwohl die Wortform eine zentrale Rolle für die Kasusmarkierung im Diasystem spielt.

Die übrigen Wortarten weisen sowohl vollständige paradigmatische Reihen als auch $\mathrm{zu}$ bestimmten Anteilen Kasusdistinktion auf. Wie angesichts der Erkenntnisse in 4.2.3 zu erwarten, zeigen die Wortarten in der 1. Person sowie solche in der 3. Person Maskulinum besonders hohe Distinktionsquoten. Hier sind insbesondere die eher suppletiven Wortarten, wie das Personalpronomen, der (maskuline) Definitartikel, aber auch das eher affigierte (maskuline) Demonstrativpronomen zu nennen.

Die Grenze zwischen eher kanonischen (zentralen) und weniger kanonischen (peripheren) Kasusmarkern wird durch eine statistische Analyse ${ }^{87}$ bestimmt: Sind

87 Ausgewertet wurde eine Tabelle mit den jeweiligen Belegmengen für die Werte der Variable Kasusdistinktion (SYNKRETISCH/ DISTINKT) für die einzelnen kasustragenden Wortformen mit einem Pearsons' $\chi^{2}$-Test $(\mathrm{Chi}=7856.6, \mathrm{df}=22, \mathrm{p}$-Wert $<2.2 \mathrm{e}-16)$. Eine vollständige Übersicht über die Residualwerte findet sich in Anhang 6.2.3. 
die Wortformen signifikant mit distinkter Kasusmarkierung assoziiert, werden sie als zentral gewertet. Zeigen sie zwar einen Anteil an Kasusdistinktion, sind aber signifikant mit synkretischer Kasusmarkierung assoziiert, zählen sie als peripher. Die so als zentral klassifizierten Kasusmarker sind in Tab. 25 mit einem *-Symbol markiert. Es handelt sich hier um die Wortformen, die neben einer vollständigen paradigmatischen Reihe auch die höchste Distinktionsquote aufweisen und für die größtenteils auch das Muster N/A/D angenommen werden kann: das Personalpronomen der 1. Person Singular sowie das Personalpronomen, der Definitartikel und das Demonstrativpronomen der 3. Person Singular Maskulinum. Lediglich das Personalpronomen der 1. Person Plural fällt zwar in diese Kategorie, neigt jedoch nicht zum distinkten Muster N/A/D, sondern zeigt ausschließlich das Muster N/AD. Der hohe Anteil an distinkten Formen lässt sich über die allgemein hohe Frequenz von Nominativbelegen im Korpus (vgl. 4.2.1) sowie hier im Speziellen über eine vermutlich textsortenspezifische Neigung zur nominativischen Selbstreferenz erklären: Die Transkripte enthalten zum Großteil Erzählungen, die Erlebnisse und Gegebenheiten aus SprecherInnenperspektive wiedergeben.

Die Wortarten, die signifikant mit Synkretismus assoziiert und mit einem ${ }^{\circ}$-Symbol gekennzeichnet sind, werden zu den peripheren Kasusmarkern gezählt. Die Wortarten, die in der Tabelle kein Symbol tragen, weisen weder eine signifikante Assoziation mit distinkter noch mit synkretischer Kasusmarkierung auf. Sie lassen sich anhand des statistischen Tests weder den zentralen noch den peripheren Markern zuordnen.

In 3.3.6 wurde die Hypothese aufgestellt, dass Frequenz einen gewissen Einfluss auf die Kasusmarkierung übt. In 4.2.1 wurde gezeigt, dass ein solcher Einfluss sich nicht über die allgemeine Tokenfrequenz der abstrakten Kasus nachweisen lässt. Die folgende Abbildung zeigt jedoch, dass auf Ebene der Kasusmarker durchaus ein Zusammenhang zwischen hoher Tokenfrequenz und einer zentralen Rolle der kasustragenden Wortform für die Kasusdistinktion im Diasystem zu erkennen ist.

Abbildung 25 listet die verschiedenen kasustragenden Wortformen gegliedert nach ihrer jeweiligen Tokenfrequenz auf. Zentrale Kasusmarker nehmen dabei tatsächlich zum größten Teil die ersten, immer aber die oberen Plätze dieser Liste ein, gefolgt von den peripheren Kasusmarkern, die über den gesamten, sehr breiten mittleren Bereich verteilt sind. Die kasustragenden Wortarten, die nicht als Kasusmarker gewertet werden, nehmen fast ausnahmslos die unteren Plätze der Liste ein, zeigen damit eine besonders niedrige Tokenfrequenz. Einzige Ausnahme bildet hier das Personalpronomen der 3. Person Singular Neutrum es, das zwar keinen Kasusmarker darstellt, jedoch relativ frequent im Korpus auftritt. Diese Wortart tritt im Korpus jedoch häufig in sehr speziellen Kontexten (z. B. als expletives es) auf, was die Sonderposition hier erklärt. 


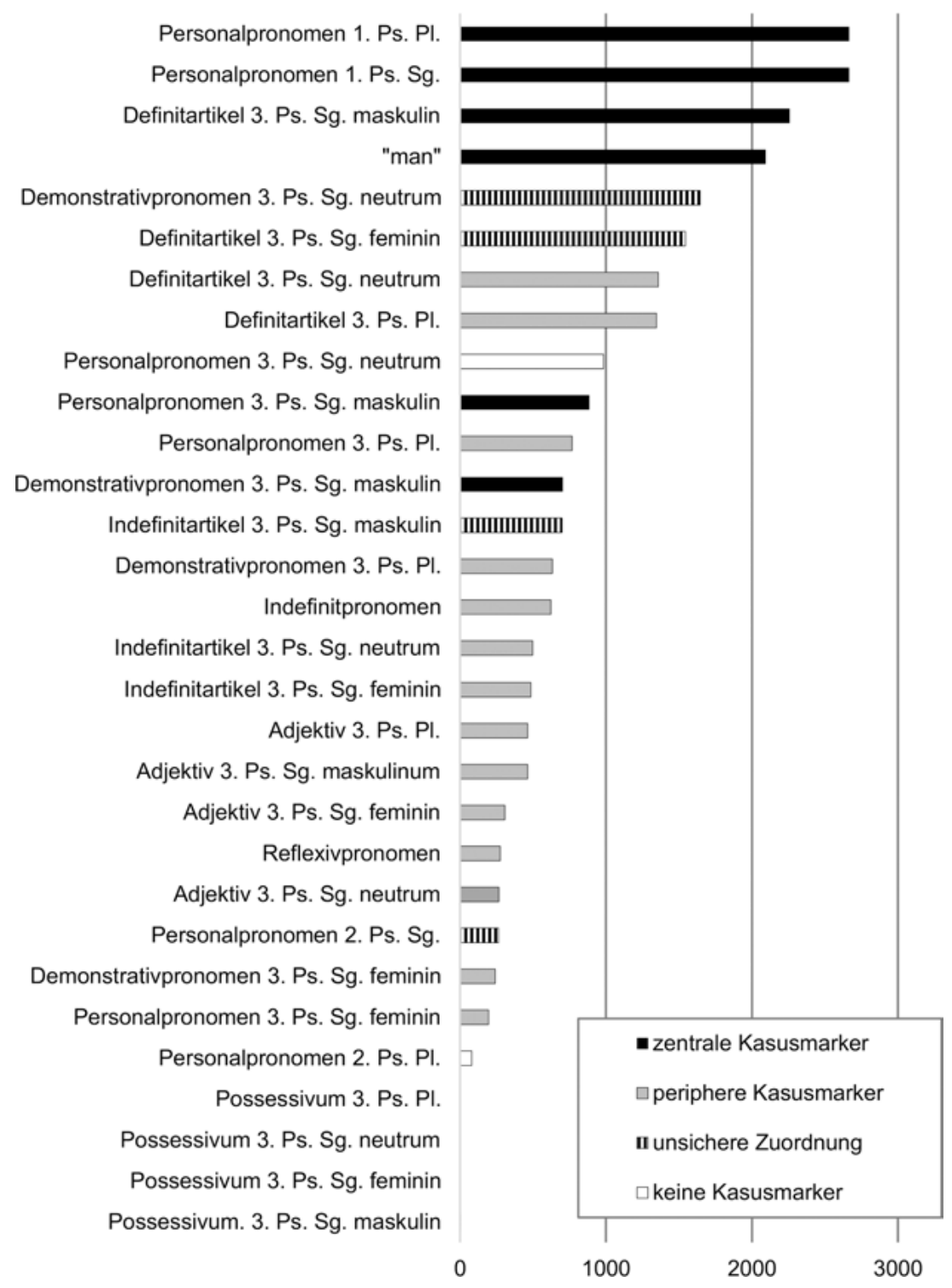

Abb. 25: Kasustragende Wortarten sortiert nach ihrer Tokenfrequenz und gegliedert nach ihrer Rolle in der Kasusdistinktion. ${ }^{88}$

88 Grundlage für diese Tabelle bildet nicht wie in Tab. 25 die Gesamtmenge der als synkretisch oder distinkt klassifizierten Belege, sondern die Gesamtmenge aller Belege der jeweiligen Wortart im Korpus. 
Hochfrequente Wortarten nehmen damit eine zentralere Rolle in der Kasusmarkierung im Diasystem ein als niedrigfrequente - ein Zusammenhang, der sich womöglich durch die diachrone Entwicklung erklären lässt: Bybee (1985: 57) geht davon aus, dass hochfrequente Wortformen eher separat gelernt werden und damit kognitiv als eigenständige Repräsentation und nicht unbedingt als Teil eines Paradigmas repräsentiert sind. Dies führt sie als Argument dafür an, dass hochfrequente Formen weniger von Wandelprozessen beeinflusst sind: „Frequency [. . .] determines the ability of a form to resist morpho-phonemic change“ (Bybee 1985: 57). In Bezug auf die Kasusdistinktion im Diasystem würde das bedeuten, dass die zentraleren Kasusmarker aufgrund ihrer hohen Tokenfrequenz diachron weniger von den Prozessen der Kasusnivellierung (vgl. 2.2.2.1) betroffen waren. Diese Hypothese wird durch die Beobachtung gestützt, dass zentralere Kasusmarker auch größtenteils suppletivere Typen der Kasusmarkierung aufweisen. Für vertiefende Erkenntnisse zu diesem Zusammenhang wäre jedoch eine diachron ausgerichtete Analyse der Kasusmarkierung notwendig.

Die paradigmatisch fokussierte Analyse in diesem Kapitel hat gezeigt, dass die Kasusmarkierung im Diasystem hauptsächlich durch Personalpronomen der 1. Person Singular und der 3. Person Maskulinum sowie durch maskuline Definitartikel und Demonstrativpronomen gewährleistet wird. Diese zentralen Kasusmarker zeigen eher suppletive Typen der Kasusmarkierung und sind größtenteils hochfrequent. Die Analyse hat aber auch dokumentiert, dass ein Großteil der hier als peripher klassifizierten Kasusmarker zu einem nicht zu unterschätzenden Teil zur Kasusdistinktion beiträgt - auch diese Wortarten zeigen durchaus distinkte Belege.

Eine vertiefende syntagmatische Analyse (vgl. dazu 4.4) muss an eben dieser Stelle ansetzen: Hier wäre zu klären, inwiefern zentrale und periphere Marker sowohl innerhalb einer Nominalphrase als auch auf Satzebene bei der Kasusmarkierung interagieren. Es gilt beispielsweise zu untersuchen, zu welchem Ausmaß das Diasystem Redundanzen oder auch Unterspezifizierungen bei der Kasusmarkierung zulässt und inwiefern sich dadurch möglicherweise die in der paradigmatischen Analyse ermittelten Distinktionsquoten erklären lassen.

\subsubsection{Zwischenfazit}

Die korpusbasierte Analyse der strukturellen Gliederung hat gezeigt, dass das Diasystem eine typologisch eher atypische Kombination abstrakter Kasus aufweist: Der synthetische Genitiv ist nur marginal belegt; es lässt sich jedoch ein Nominativ, ein Akkusativ und ein Dativ ansetzen (4.2.1). Der Dativ weist aufgrund seines großen Funktionsspektrums einen großen Anteil an distinkter Markierung auf und 
nimmt damit eine herausragende Position in der Distinktionshierarchie des Diasystems ein, was sich auch in den Mustern der Kasusmarkierung spiegelt: lediglich die 1. Person, ein kleiner Teil der 3. Person Maskulinum sowie das Muster NAD zeigen synkretische Dativformen. Niedriger ist der Anteil distinkter Formen des Nominativs. Der Akkusativ weist den geringsten Anteil distinkter Formen auf. Obwohl das Muster NA/D, wo sowohl Nominativ als auch Akkusativ synkretisch sind, hochfrequent und in zahlreichen Kontexten auftritt, gleichen sich die beiden Kasus im Anteil distinkter Kasusmarkierungen deutlich weniger als erwartet, was sowohl durch die Kategorien mit Akkusativ-Dativ-Synkretismus als auch die zahlreichen und zudem äußerst frequenten Kontexte der Nominativdistinktion begründet werden konnte.

Bei der distinkten Markierung der abstrakten Kasus spielen suppletive Strukturen tatsächlich eine herausragende Rolle (4.2.2). In den Fällen, in denen affigierte Strukturen zur Kasusdistinktion beitragen, handelt es sich um solche mit eher intransparenter Affigierung, wobei sich bei diesen Typen der Kasusmarkierung im Speziellen ein Zusammenhang zwischen Formenreduktion, Suppletion und Kasusdistinktion abgezeichnet hat. Eine modifizierte Analyse auf Basis eines Korpus mit phonologisch-prosodisch exakterer Transkription könnte hier noch vertiefende Einblicke ermöglichen. Entsprechende methodische Überlegungen und erste konkrete Vorschläge für den Aufbau einer solchen Analyse wurden in 4.2.2 angeführt.

Die Muster der Kasusmarkierung sind stark von der Dependenzhierarchie zwischen Kasus und anderen grammatischen Kategorien - namentlich Genus, Numerus und Person - geprägt (4.2.3). Diese Kategorien üben nicht nur Einfluss auf die exakten Muster, sondern auch auf die Höhe der Distinktionsquote aus. Die 1. Person Singular sowie die 3. Person Maskulinum zeigen besonders viel Kasusdistinktion und sind die Domänen des Musters N/A/D. Der Grund für die Sonderstellung dieser Kategorien scheint ihre Assoziation mit besonders agentivischen bzw. menschlichen Referenten zu sein und damit bei semantische Regularitäten zu liegen, die über die grammatischen Kategorien Einfluss auf das Kasussystem üben.

Die Erkenntnisse zu Typen und Mustern der Kasusmarkierung spiegeln sich auch in den Wortarten wider, die die zentralen Kasusmarker im Diasystem darstellen (4.2.4). Dabei handelt es sich um eben die Wortarten, die zum einen hochfrequent sind und zum anderen eher suppletive bzw. intransparent affigierte Strukturen zeigen - Personal- und Demonstrativpronomen sowie Definitartikel -, die in der 1. Person oder im Maskulinum Singular auftreten. Daneben gibt es im Diasystem jedoch auch zahlreiche periphere Kasusmarker, die zwar keine derart hohe Distinktionsquote aufweisen wie die zentralen Marker, aber doch zu einem gewissen Anteil zur Kasusdistinktion beitragen. Methodisch 4.2.4 dabei gezeigt, 
dass das Korpus teilweise keinen vollkommen homogenen Ausschnitt aus dem Kasusdiasystem abbildet: So treten manche Wortarten besonders niedrigfrequent auf, wodurch sich keine vollständige paradigmatische Reihe rekonstruieren lässt, bei manchen ist eine bestimmte paradigmatische Zelle überrepräsentiert. Die transparente Übersicht über die zugrundeliegenden Daten hat sich in diesen Fällen als äußerst hilfreich erwiesen.

Neben einem Einblick in die strukturelle Gliederung des Kasusdiasystems sowie in die Datenstruktur des Korpus hat dieses Kapitel die Grundlage für die folgende dialektgeographische Analyse geschaffen: Angelehnt an die strukturelldialektologische Tradition wurden zunächst die übergreifenden Muster und Strukturen abgebildet, die das Diasystem als solches konstituieren. Variation, die sich hier bereits angedeutet hat, wird im folgenden Kapitel auf ihre geographische Determination hin untersucht. Die Kategorien hingegen, die keine Variation zeigen, können als teilsystemübergreifend und damit als die Faktoren gewertet werden, die das untersuchte Diasystem konstituieren.

\subsection{Geographische Gliederung des Untersuchungsgebietes}

In 2.3 wurde das Forschungsfeld der dialektgeographischen Kasusmorphologie bereits ausführlich skizziert. Es hat sich dabei als bislang noch weniger erforschter Bereich erwiesen, in dem zahlreiche weiße Flecken existieren, die es noch zu erkunden gilt. Dieses Kapitel hat zum Ziel, einen Vorstoß in das Feld der kasusmorphologischen Raumgliederung $\mathrm{zu}$ wagen und damit zumindest einen Teil der offenen Fragen aufzugreifen und auf Grundlage der hier analysierten Daten zu beantworten. Dabei werde ich mich auf die geographische Gliederung der Muster der Kasusmarkierung konzentrieren - einen Aspekt, zu dem zwar insbesondere durch Shrier (1965) bereits erste Erkenntnisse existieren, die jedoch speziell im Untersuchungsgebiet der Korpusanalyse auf groben Abstraktionen einer recht kleinen Datengrundlage beruhen und daher noch viel Raum zur strukturellen Vertiefung und geographischen Verdichtung bieten. Dabei werde ich induktiv vorgehen und die kasusmorphologische Raumgliederung auf Basis der Korpusdaten sowie ergänzend auf Grundlage der Ortsgrammatiken bestimmen.

Insbesondere ein Blick zur in den letzten Jahren recht ausführlich untersuchten räumlichen Gliederung syntaktischer Phänomene zeigt hier interessante Parallelen auf und bietet nützliche Beschreibungsmodelle, die auch an die kasusmorphologische Raumgliederung angelegt werden können. Den Ausgangspunkt bilden die Muster der Kasusmarkierung in maskulinen Wortarten, die sich im Verlauf des Kapitels als raumbildende Kategorien herauskristallisieren. 
Basierend darauf werden generelle Strukturen der kasusmorphologischen Raumbildung herausgearbeitet und mit allgemeinen Erkenntnissen der morphosyntaktischen Dialektgeographie in Bezug gesetzt. Inwiefern diese Modelle vollständig auf die geographischen Strukturen der Kasusmorphologie übertragen werden können, beziehungsweise an welchen Stellen sie unter Umständen ergänzt oder verändert werden müssen, um die Komplexität der Kategorie Kasus zu erfassen, wird im Verlauf des Kapitels zur Diskussion gestellt.

\subsubsection{Raumbildende Kategorien und konstituierende Faktoren des Diasystems}

In 4.1 wurden die verschiedenen Kasusmarker bereits ausführlich abgebildet. Abstrahiert von möglicher geographischer Variation wurden Muster und Strukturen der Kasusmarkierung dort zusammenfassend für das gesamte Diasystem dort beschrieben. In diesem Teilkapitel stehen nun die Kasusmarker und Muster im Vordergrund, die das Diasystem in Teilsysteme gliedern und dabei geographische Distribution aufweisen: die raumbildenden Kategorien der Kasusmarkierung im Untersuchungsgebiet.

Im untersuchten Diasystem können aufgrund der geographischen Nähe der einzelnen Erhebungsorte eng verwandte Teilsysteme erwartet werden, d. h. solche, die sich nur in den Mustern weniger Kasusmarker unterscheiden. Die Kategorien, deren Muster keine Variation im Diasystem zeigen, die das Diasystem nach der Tradition der strukturellen Dialektologie als solches konstituieren, überwiegen hier demnach deutlich. Kapitel 4.2.3 führt keine Variation bei den Mustern im Plural (N/AD in der 1. und NA/D in der 3. Person) an. Diese sind demnach im gesamten Gebiet identisch. Auch die starke Stellung des (distinkten) Dativs, die sich sowohl in zahlreichen Mustern der Kasusmarkierung als auch im großen Anteil der distinkten Dativmarkierung abzeichnet, kann als konstituierender Faktor des Diasystems gewertet werden, ebenso wie die hohe Synkretismusquote beim Akkusativ.

Geographische Variation wird nun grundsätzlich nur bei den Kategorien erwartet, die in der Übersicht 4.2.3 mehrere Muster der Kasusmarkierung zeigen. Dabei ist jedoch nicht jede Kategorie, die mehr als ein Muster aufweist, raumbildend. Variation innerhalb der Muster einer Kategorie kann in den Korpusdaten neben der geographischen Distribution der Teilsysteme auch andere Ursachen haben. Auch eine diasystemübergreifende strukturelle Variation, beispielsweise durch Vertikalisierungstendenzen, ist möglich. Ebenso sind Artefakte von Transkription oder Datenanalyse denkbar. So lässt sich beispielsweise vermutlich der (ohnehin recht geringe) Anteil des totalen Synkretismus beim Femininum (8,1\%) 
und Neutrum (2,2\%) oder auch der (verschwindend geringe) Anteil des Musters N/AD (0,09\%) im Femininum erklären.

Das Maskulinum zeigt gegenüber den anderen Kategorien ein besonders breites Variationsspektrum. Alle möglichen Muster (N/A/D 70,8\%, NA/D 18,9\%, N/AD 7,1\% und NAD 3,2\%) werden im Untersuchungsgebiet tatsächlich umgesetzt, ein Umstand, der auf Grundlage der Literatur durchaus zu erwarten war. Shrier (1965: 431-435) zieht das Maskulinum als primäre Grundlage zur kasusmorphologischen Gliederung des gesamten deutschsprachigen Gebiets heran und auch die übrigen in 2.3 angeführten Arbeiten legen einen starken Fokus auf diese Kategorie. Die Korpusdaten bestätigen damit die zentrale Rolle der maskulinen Kategorien bei der kasusmorphologischen Raumgliederung im oberdeutschen Gebiet.

Doch auch eine weitere Kategorie weist in den Daten eine gewisse Mustervariation auf. Die 1. Person Singular zeigt neben dem dominanten Muster N/A/D auch vereinzelt das dort für den Plural charakteristische Muster N/AD. Verglichen mit dem dem Anteil der Mustervariation im Maskulinum ist der Anteil der Belege dieses abweichenden Musters (das in Shrier 1965: Karte 13 zudem nicht angeführt wird) zwar mit 5,2\% äußerst gering, doch wäre eine strukturelle und damit auch geographische Ausbreitung gerade dieses Musters in der 1. Person Singular keineswegs abwegig. Eine Ausdehnung des charakteristischen Musters der 1. Person vom Plural auf den Singular analog zum Prozess bei der 3. Person wäre ein durchaus realistisches Szenario.

In den folgenden Kapiteln gilt es daher erstens zu untersuchen, inwiefern die in maskulinen Kategorien, aber auch bei der 1. Person Singular im Korpus nachgewiesene Mustervariation tatsächlich geographisch determiniert ist, wie zweitens die geographischen Muster den Raum gliedern und ob es drittens womöglich weitere Faktoren neben der geographischen Determination gibt, die Mustervariation bestimmen.

\subsubsection{Daten zur Raumgliederung anhand maskuliner Kategorien}

Während sich die Analyse bislang auf die Daten der frequenzbasierten Korpusanalyse konzentriert hat, werden nun zur Darstellung der kasusmorphologischen Raumgliederung die Erkenntnisse aus der Untersuchung der Ortsgrammatiken mit denen der Korpusanalyse kombiniert. Die unterschiedlichen Eigenschaften der Daten bieten dabei die Möglichkeit, verschiedene Aspekte der dialektgeographischen Gliederung des untersuchten Kasusdiasystems zu beleuchten: Das dichte Ortsnetz der Korpusbelege ermöglicht die detaillierte Analyse eines kleinen Untersuchungsgebiets. Verglichen damit ist das Ortsnetz der Ortsgrammatiken 
nicht besonders dicht, deckt jedoch ein deutlich größeres Gebiet, das nahezu den gesamten oberdeutschen Sprachraum umfasst, ab. Es ermöglicht die Einbettung der Ergebnisse zur Gliederung der Korpusdaten in großräumigere Strukturen.

Aufgrund des bereits in 3.2.2 angesprochenen kanonisierten Aufbaus der Ortsgrammatiken können für nahezu jeden Ort vollständige Paradigmen zu diversen kasustragenden Wortarten abgebildet werden. Damit liegt hier ein sehr dichtes Belegnetz, d. h. eine sehr homogene Datenstruktur vor (vgl. dazu 3.2.2). Die Daten der Korpusanalyse sind in diesem Punkt deutlich heterogener. Abhängig von Länge und Inhalt des Redebeitrags sowie von der Gebrauchsfrequenz der jeweiligen Wortart variiert die Belegzahl an den Ortspunkten so stark, dass an einzelnen Erhebungspunkten für bestimmte Kasusmarker kein vollständiges Paradigma rekonstruiert werden kann. Trotz des relativ dichten Ortsnetzes ist hier folglich mit gewissen strukturellen Lücken in der geographischen Abbildung der Muster und damit mit einem heterogenen Belegnetz $\mathrm{zu}$ rechnen.

Zudem ist ein Unterschied in den abgebildeten Mustern der Kasusmarkierung zwischen den beiden Datengrundlagen denkbar, der auf diachrone Wandelprozesse im Kasusdiasystem zurückzuführen ist. Es ist davon auszugehen, dass die Ortsgrammatiken, die vorwiegend Nennformen und damit wahrscheinlich auch Erinnerungsformen auflisten, einen eher älteren Sprachstand abbilden als die Korpusdaten, die gesprochene Sprache und damit die aktive Kompetenz der SprecherInnen wiedergeben. Bezogen auf ihren Erhebungszeitpunkt decken die Ortsgrammatiken zwar ohnehin insgesamt einen deutlich größeren Zeitraum ab als die Korpusdaten; der Großteil der Grammatiken ist jedoch vor den Tonaufnahmen zu Ruoff (1984) entstanden (vgl. dazu die Übersicht der zeitlichen Einordnung der Daten in Abb. 26). Dabei handelt es sich um einen zusätzlichen Aspekt, der dafür spricht, dass in den Ortsgrammatiken ein älterer Sprachstand abgebildet wird. Bei direktem Vergleich von Mustern der Kasusmarkierung in einzelnen Orten und Gebieten in beiden Datengrundlagen muss diese Chronologisierung in jedem Fall berücksichtigt werden. Die folgende Kartierung der Daten rekonstruiert demnach einen synchronen Zustand auf Grundlage eines diachronen Datenspektrums, das in Abb. 26 dargestellt ist. 


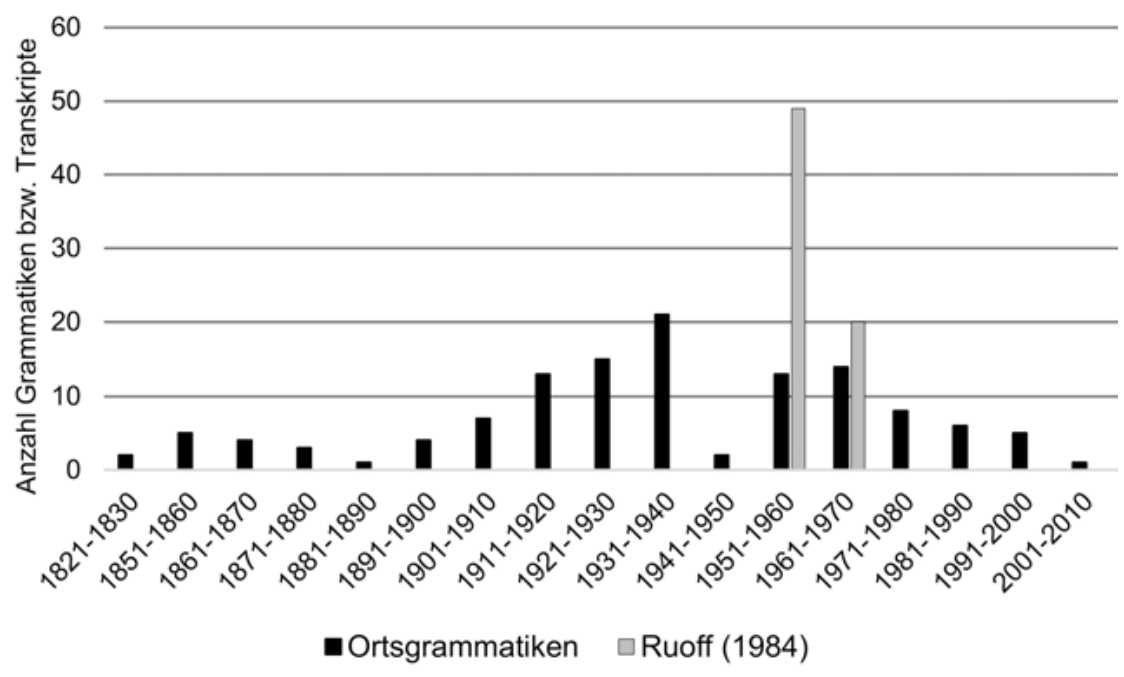

Abb. 26: Zeitliche Einordnung der Datengrundlagen nach Erhebungs- bzw. Publikationsdatum.

Wie bereits in 4.3.1 angeführt wurde, werden primär maskuline Wortarten als raumbildende Kategorien angesetzt. Im Folgenden werde ich daher zunächst die geographischen Raumbilder aufgeteilt nach den einzelnen maskulinen Wortarten in Karten darstellen, danach auf allgemeinere Gliederungsfaktoren, die sich in dieser Darstellung zeigen, eingehen und abschließend einen kurzen Überblick über die Muster des Personalpronomens der 1. Person Singular im Raum geben. Zunächst werde ich mich dabei auf den Untersuchungsraum der Korpusanalyse konzentrieren.

Die Karten zu den Korpusdaten sind wie folgt gestaltet: Die einzelnen Farben innerhalb der Kuchendiagramme weisen auf verschiedene Muster der Kasusmarkierung hin. Enthält ein Diagramm mehr als eine Farbe, konnte Variation zwischen den einzelnen Mustern festgestellt werden. In 4.3.4 wird auf diese Variation explizit eingegangen. Die Legende führt zudem jeweils die absolute Belegmenge an, auf deren Basis das jeweilige Muster der Kasusmarkierung rekonstruiert wurde. Die Größe der Kuchendiagramme richtet sich nach der relativen Belegzahl, die der automatisierten Analyse von Mustern der Kasusmarkierung für die entsprechende Wortart am jeweiligen Ort zur Verfügung standen. An Orten, an denen ein graues Punktsymbol abgebildet ist, sind nicht genug Belege vorhanden, um ein Muster zu rekonstruieren. An den Orten, an denen zwei Transkripte vorhanden sind, ist das kleinere Diagramm vor dem größeren abgebildet. 
Abbildung 27 zeigt die Ergebnisse zur geographischen Gliederung des maskulinen Adjektivs. Dort fällt zunächst das unvollständige Belegnetz auf, das bereits oben als typisches Merkmal einer Korpusanalyse gesprochener Sprache wurde. Es konnten nicht für jeden, aber doch für knapp die Hälfte der untersuchten Orte Muster konstruiert werden. An den übrigen Orten zeigen sich ausschließlich synkretische Muster. Die volle Distinktion N/A/D, die eigentlich das dominante Muster maskuliner Kategorien darstellt (vgl. 4.2.3), tritt hier in keinem Teilsystem auf, was auch die relativ niedrige Distinktionsquote des (maskulinen) Adjektivs erklärt (vgl. Tab. 25), das mit 25\% die mit Abstand niedrigste Quote aller maskulinen Kasusmarker zeigt. Stattdessen finden sich Belege für den totalen Synkretismus NAD (z. B. nennenswärda, alda, erschda, Donaustetten/Ulm), das Muster NA/D (z. B. hendre, kloine/ letschta, Dillingen/Frauenriedhausen) sowie am häufigsten solche für N/AD (z. B. guter/ erschta, falscha, Mindelaltheim/Günzburg). Eine Raumbildung ist hier nur schwer zu erkennen; lediglich eine relativ homogene Verbreitung des N/AD-Musters im Süden und Osten sowie eine Konzentration des Musters NAD im Zentrum des Gebiets können hier angenommen werden.

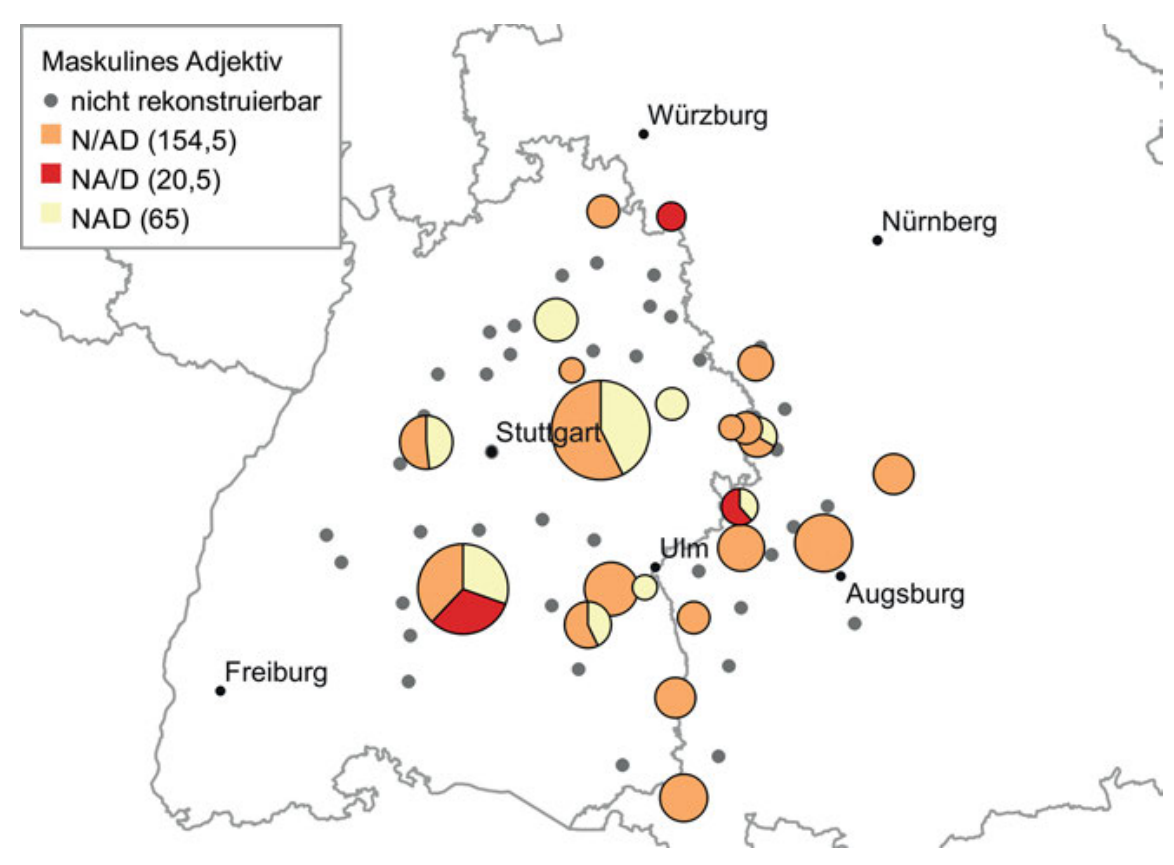

Abb. 27: Kasusmorphologische Raumbildung anhand der Korpusdaten zum maskulinen Adjektiv. 
Dieses Ergebnis ist beim Adjektiv nicht unbedingt verwunderlich: Bereits in 4.2.3 konnte diese Wortart als peripherer Kasusmarker klassifiziert werden. Sie bildet zwar eine paradigmatische Reihe, weist aber genusübergreifend einen relativ hohen Synkretismusanteil auf, wodurch mit einem großen Anteil an distinkten Belegen nicht $\mathrm{zu}$ rechnen war. Wie bereits in 4.2.2 ausgeführt wurde, stellt die wortbasierte Ausrichtung der Analyse zudem eine gewisse Hürde für die Klassifikation der Adjektive dar, da diese häufig transparente Flexion aufweisen (was eher eine morphembasierte Analyse verlangen würde) und zudem, verglichen mit anderen kasustragenden Wortarten, zu starker lexikalischer Variation neigen. Die daraus resultuierende verhältnismäßig große Heterogentität der Daten kann hier unter Umständen zu einem weniger eindeutigen Kartenbild geführt haben.

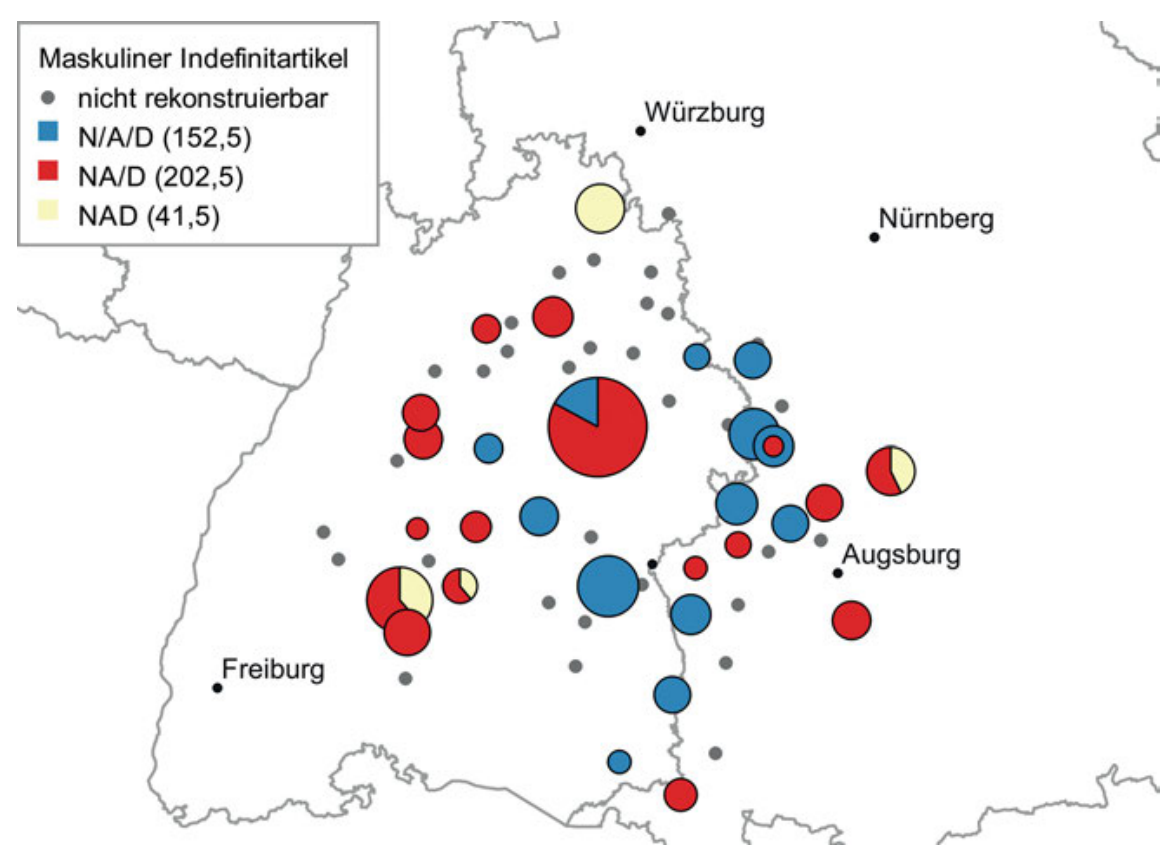

Abb. 28: Kasusmorphologische Raumbildung anhand der Korpusdaten zum maskulinen Indefinitartikel.

Ganz anders als beim maskulinen Adjektiv stellt sich die geographische Gliederung des Untersuchungsgebiets anhand des maskulinen Indefinitartikels in Abb. 28 dar. Zwar zeigt die Karte auch hier nicht für jeden der Ortspunkte ein Muster der Kasusmarkierung, doch sind die Ergebnisse hier deutlich gleichmä- 
Biger über das Gebiet verteilt als beim Adjektiv. Auch die Muster selbst unterscheiden sich von denen des Adjektivs. Das dort dominante Muster N/AD tritt hier nicht auf. Dagegen zeigt sich ein recht ausgewogener Anteil an voller Distinktion (z. B. a/ en/ ma, Altheim/Ulm) und dem Muster NA/D (z. B. an, an / ma, Heimsheim/Leonberg). An einzelnen Ortspunkten tritt auch Variation zwischen diesen beiden Mustern auf. Der totale Synkretismus NAD (z. B. an, an, an, Ballersdorf/Neuburg) kommt nur punktuell vor. Die Belege der vollen Distinktion zeigen eine gewisse geographische Bündelung im zentralen und südöstlichen Bereich des Untersuchungsraums. Lediglich am südlichen und östlichen Rand und im Westen zeigen sich die Belege für das Muster NA/D. Im Norden konnte für ein größeres Gebiet kein Muster rekonstruiert werden.

Insbesondere die Belege des Musters NA/D im Süden und Osten des Untersuchungsgebiets unterscheiden sich hier von den Annahmen, die auf Basis der bisherigen Forschungsliteratur (2.3.4), namentlich auf Basis von Shrier (1965), getroffen werden konnten. Wie in Abb. 6 dargestellt, wurde dieses Muster dort lediglich für den westlichen Rand des Untersuchungsgebiets prognostiziert. Im Süden wurde hier ausschließlich die volle Distinktion und am Ostrand das Muster N/AD angesetzt (vgl. dazu Abb. 7). Die Korpusdaten zeigen folglich ein deutlich größeres Verbreitungsgebiet des Musters NA/D. Hierbei kann es sich sowohl um eine auf das lose Ortsnetz zurückzuführende Ungenauigkeit in den Karten von Shrier (1965) als auch um einen durch diachron zunehmende Kasusnivellierung bedingten Unterschied zwischen den Korpusdaten und den auch von Shrier (1965) benutzten Ortsgrammatiken handeln.

Die geographische Gliederung anhand des maskulinen Indefinitartikels ist dabei einheitlicher als sich durch die bisherigen Erkenntnisse zur Kasusdistinktion beim Indefinitartikel hätte erwarten lassen. Das breite Spektrum der morphologischen Strukturen, das hier unter anderem auch durch Formenreduktion geprägt ist (vgl. 4.2.2), hätte durchaus eine größere und nicht unbedingt raumbildende Mustervariation hervorrufen können. Dennoch zeigt sich insbesondere das Gebiet der vollen Distinktion relativ eindeutig in den Korpusdaten - eine Erkenntnis, die durchaus als Argument für die morphologische Zuverlässigkeit der Transkription in Ruoff (1984) gewertet werden kann.

Der maskuline Indefinitartikel konnte anhand des $\chi^{2}$-Tests in 4.2.4 weder der Gruppe der zentralen, noch der der peripheren Kasusmarker zugeordnet werden. Verglichen mit dem maskulinen Adjektiv, das als peripherer Kasusmarker klassifiziert wurde, zeigt er im Untersuchungsgebiet jedoch einen deutlich geringeren Anteil an synkretischen Mustern und ein Kerngebiet der vollen Distinktion N/A/D, was sich auch in seiner höheren Distinktionsquote (47\%) widerspiegelt. Damit deutet sich beim Indefinitartikel eine geographische Struktur an, die sich im Folgenden als charakteristisch für die zentralen Kasusmarker im Untersuchungsgebiet 
der Korpusanalyse erweisen wird: ein Distinktionsgebiet, das im Zentrum des Untersuchungsraums verankert ist und wo Kasusnivellierung weder durch das Muster N/AD noch durch das ansonsten in der 3. Person dominante Muster NA/D auf die zentralen maskulinen Kasusmarker übergegriffen hat.

Besonders klar zeigt sich diese spezielle Struktur der geographischen Gliederung in Abb. 29 zum maskulinen Definitartikel. Die volle Distinktion N/A/D (z. B. $d r /$ en/ em, Alfdorf/Schwäbisch Gmünd) dominiert hier, gefolgt vom Muster NA/D (z. B. däär, däär/ am, Gellmersbach/Heilbronn). Das Muster N/AD (z. B. d'r/ en, en, Hengstfeld/Crailsheim_2) tritt zwar nicht derart häufig wie die beiden anderen Muster auf, zeigt aber eine relativ klare Raumbildung im Osten und Nordosten des Gebiets. Mit Ausnahme dieses kleineren Raums dominiert die volle Distinktion überall; ein geschlossenes Distinktionsgebiet, in dem ausschließlich N/A/D auftritt, bildet sie jedoch ausschließlich im Zentrum. Im Westen, im mittleren Osten sowie vereinzelt im Norden tritt neben der vollen Distinktion auch verstärkt das Muster NA/D auf. An den Rändern des Distinktionsgebiets findet sich an einzelnen Orten Variation zwischen N/A/D und den beiden synkretischen Mustern.

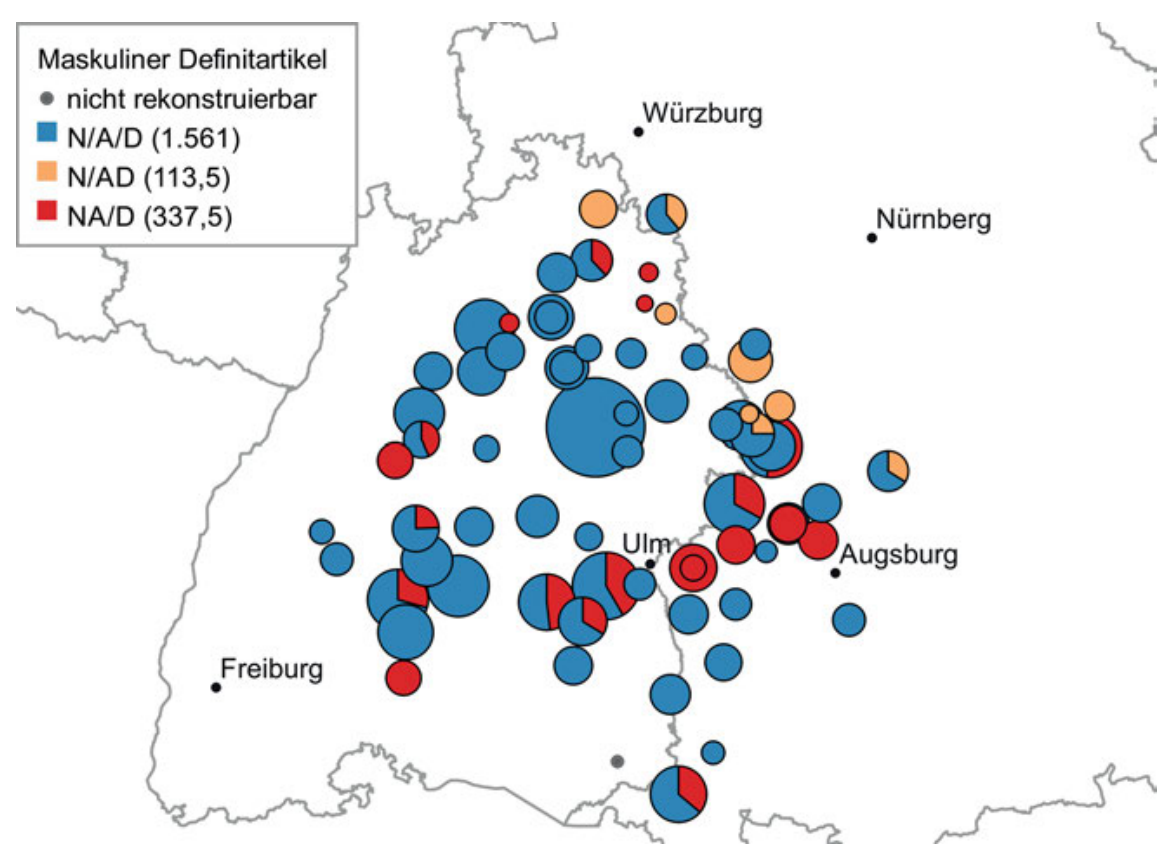

Abb. 29: Kasusmorphologische Raumbildung anhand der Korpusdaten zum maskulinen Definitartikel. 
Wie bereits beim Indefinitartikel unterscheiden sich auch die Ergebnisse beim Definitartikel im Korpus von den bisherigen Erkenntnissen: Hier findet sich das Muster NA/D zwar tatsächlich am westlichen Rand des Untersuchungsgebiets, aber zudem auch im Norden, im Süden sowie im Südosten. Lediglich im Zentrum, wo ausschließlich volle Distinktion auftritt, und im Nordosten, wo das in Shrier (1965) für den östlichen Rand prognostizierte Muster N/AD vorkommt, gibt es keine Belege für NA/D. Dieses Muster zeigt sich in den Korpusdaten damit nicht nur deutlich weiter östlich als in Shrier (1965) angesetzt. Auch das bereits in Rowley (2004: 357) mithilfe von Korpusdaten nach Osten erweiterte Gebiet erfasst nicht die vollständige Verbreitung dieses Musters.

Das Muster NA/D lässt sich - zumindest in der an der Systemfunktion orientierten Verwendungsweise von Rowley (2004) - schwerlich mit dem Terminus „rheinischer Akkusativ“ bezeichnen. Die Korpusdaten zeigen, dass das Verbreitungsgebiet dieses Musters deutlich über den alemannischen Raum entlang des Rheins, den Hildebrand (1869) ursprünglich als Namensgeber und zur geographischen Verortung für dieses Muster gewählt hat, hinausgeht. Weshalb der Terminus für das Muster NA/D nach wie vor recht prominent ist, zeigt ein kurzer Blick auf die entsprechende Karte, die auf Grundlage der Ortsgrammatiken erstellt wurde: Abb. 35 zeigt das Muster NA/D tatsächlich primär im alemannischen Gebiet und entlang des Rheins. Lediglich im Norden des oberdeutschen Raums beziehungsweise im rheinfränkischen Gebiet ist das Muster auf dieser Karte belegt. Für den Bereich im Zentrum und im Südosten des Untersuchungsgebiets der Korpusanalyse, wo hier zusätzliche Belegräume nachgewiesen werden konnten, zeigt die Karte jedoch eine große Lücke im Ortsnetz, einen weißen Fleck in der Analyse der Ortsgrammatiken. Da es sich dabei bislang um die primäre Datenquelle der kasusmorphologischen Dialektgeographie handelt, ist es nicht unbedingt verwunderlich, dass das Muster bis jetzt ausschließlich am Rhein und nicht - um bei der Flussreferenz zu bleiben - auch an der Donau angesetzt wurde.

Wie bereits oben in Bezug auf den Indefinitartikel erwähnt, ist das klare Raumbild beim maskulinen Definitartikel unter Berücksichtigung der verschiedenen Reduktionsformen, die hier auftreten und durchaus zu Unterschieden in der Systemfunktion führen können (vgl. 4.2.2), erstaunlich. Die hohe Frequenz des Definitartikels im Korpus (allein im Maskulinum 2.258 Belege) gewährleistet jedoch eine besonders dichte Abdeckung des Untersuchungsraums. Lediglich für das Teilsystem an einem einzelnen Ort konnte kein Muster rekonstruiert werden. Der maskuline Definitartikel ist daher auch aus rein methodologischen Gründen geradezu prädestiniert für die Analyse der dialektgeographischen Gliederung des Untersuchungsraums, was sicher auch den starken Fokus kasusmorphologischer Untersuchungen auf diese Kategorie erklärt (vgl. Meyer 1967, Dal Negro 2004, Studler 2013, Perrig 2018 uvm.). 
Für das maskuline Demonstrativpronomen lassen sich keine Vermutungen aus der Forschungsliteratur heranziehen. Shrier (1965) bindet diese Wortart weder in ihre Karte zum Überblick über synkretische Muster im Maskulinum (Abb. 6) noch zur vollen Distinktion (Abb. 7) ein. Auch die Ergebnisse der Korpusanalyse in Abb. 30 sind verglichen mit denen zum Definitartikel etwas ernüchternd. Die Daten reichen hier nicht aus, um ein flächendeckend einheitliches Bild der räumlichen Gliederung zu ermöglichen. Nichtsdestoweniger lässt sich erkennen, dass die volle Distinktion (z. B. där/ dean/ dem, Alfdorf/Schwäbisch Gmünd) gegenüber dem Muster NA/D (z. B. där, där/ dä(a)m, Wengen/Kempten) deutlich dominiert, was die hohe Distinktionsquote von 86\% erklärt. Im Vergleich mit Abb. 28 und 29 zeigt sich zudem, dass das Muster der vollen Distinktion beim Demonstrativpronomen auch an Ortspunkten auftritt, an denen beim Definit- und Indefinitartikel das Muster NA/D dominiert.

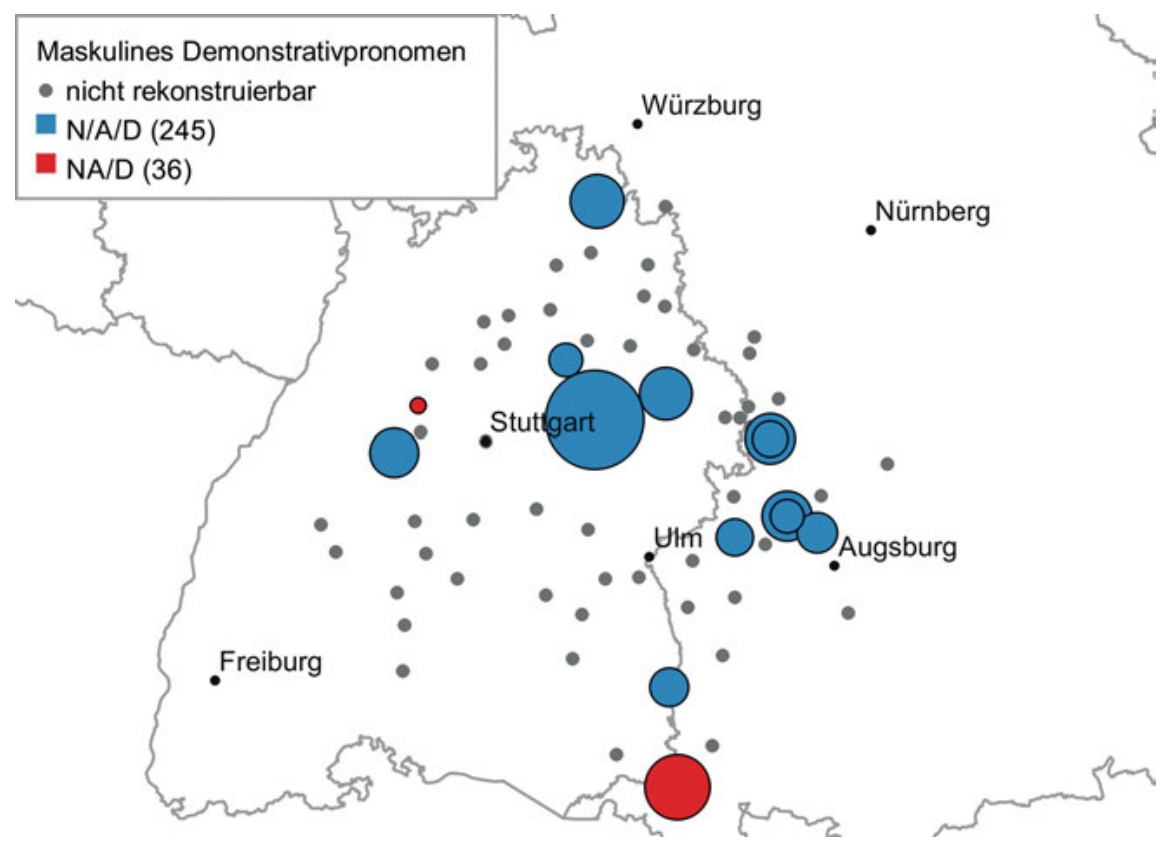

Abb. 30: Kasusmorphologische Raumbildung anhand der Korpusdaten zum maskulinen Demonstrativpronomen.

Auf Grundlage von Abb. 30 lässt sich - unter Berücksichtigung des recht dünnen Belegnetzes - vorsichtig eine großflächigere Verbreitung des zentralen Distinktionsgebiets beim Demonstrativpronomen gegenüber den bisher aufgeführten Wortarten ausmachen. 
Die Karte zeigt an den Orten, an denen ein Muster rekonstruiert werden konnte, keinerlei Mustervariation, was möglicherwiese auf das geringe Spektrum von Typen der Kasusmarkierung zurückzuführen ist (vgl. 4.2.2). Demonstrativpronomen zeigen weniger transparente Affigierung als beispielsweise Adjektive und Possessiva. Sie weisen auch keine lexikalische Variation auf und sind damit auch anhand einer wortbasierten Analyse gut zu greifen. Sie neigen jedoch auch nicht im Ausmaß der Artikel zur Formenreduktion oder gar Klise, sodass Mustervariation auf Basis wechselnder Typen der Kasusmarkierung hier nahezu ausgeschlossen werden kann.

Wie sich aus der bisherigen Forschungsliteratur bereits vermuten lässt, bildet das Personalpronomen verglichen mit den anderen maskulinen Wortarten ein besonders großes Distinktionsgebiet im Untersuchungsraum der Korpusanalyse aus. Abbildung 31 zeigt für diese Wortart zwar auch ein eher loses Belegnetz, doch finden sich hier nahezu ausschließlich Muster der vollen Distinktion (z. B. är/ $\mathrm{n} / \mathrm{em}$, Herkheim/Nördlingen), die zudem relativ gleichmäßig über das gesamte Gebiet verteilt sind. Die Ausnahme bildet hier lediglich ein einziger Ortspunkt im Osten, der das Muster N/AD (z. B. $r / n, n$, Mindelaltheim/Günzburg) zeigt.

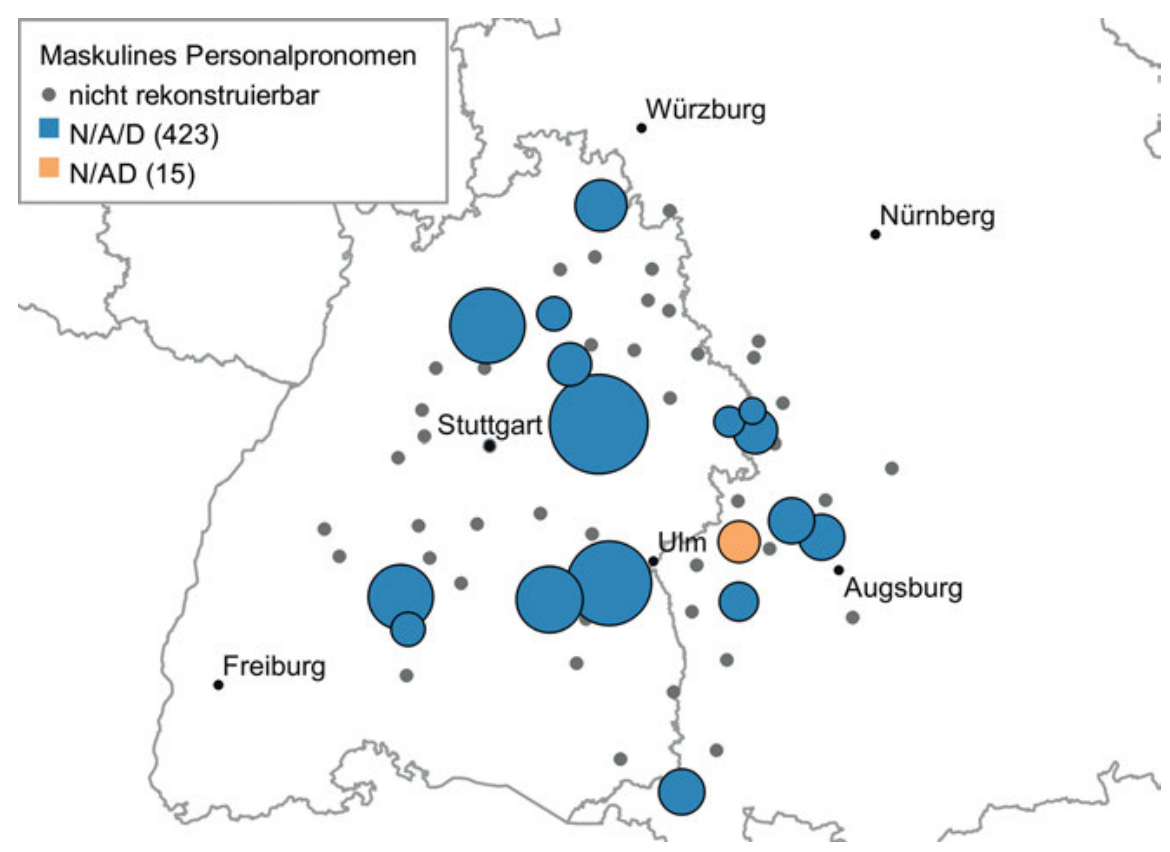

Abb. 31: Kasusmorphologische Raumbildung anhand der Korpusdaten zum maskulinen Personalpronomen. 
Abbildung 31 spiegelt im Vergleich zu den zuvor aufgeführten Karten die bereits mit Howe (1996: 70-71) angeführte Beobachtung wider, dass Personalpronomen als Kategorie mit besonders hohem Anteil der Kasusdistinktion zu werten sind. Sie zeigt, dass diese hohe Distinktionsquote der Personalpronomen im Diasystem nicht nur mit einem strukturell hohen Anteil, sondern auch mit einem geographisch besonders großen Gebiet der vollen Distinktion einhergeht.

Die geographische Gliederung, die auf Basis der zentralen maskulinen Kasusmarker im Untersuchungsgebiet der Korpusanalyse abgebildet werden kann, lässt sich damit wie folgt beschreiben: Im Zentrum befindet sich ein Gebiet, in dem das Muster der vollen Distinktion erhalten ist. Im Westen und Süden wird es vom synkretischen Muster NA/D begrenzt; im Osten und teilweise Nordosten vom synkretischen Muster N/AD. Die geographische Ausdehnung des synkretischen Musters NA/D in das Distinktionsgebiet lässt sich kategoriell entlang der Wortarten im Maskulinum staffeln. Während sich beim maskulinem Adjektiv als peripherem Kasusmarker flächendeckend synkretische Muster zeigen, zeichnet sich ein Kerngebiet der vollen Distinktion um den südlichen Teil der im Untersuchungsgebiet gelegenen politischen Grenze der Bundesländer Baden-Württemberg und Bayern ab, wo sich das Muster noch beim Indefinitartikel zeigt und von dem aus sich die Distinktion N/A/D insbesondere nach Norden und Westen, teilweise aber auch nach Osten hin ausdehnt. Die folgende Skala illustriert diese kategorielle Staffelung der geographischen Verbreitung des Distinktionsgebiets entlang der maskulinen Wortarten.

(12) Indefinitartikel < Definitartikel < Demonstrativpronomen $<$ Personalpronomen

Dabei lässt sich die kategorielle Staffelung des Distinktionsgebiets analog zur Distinktionsquote der einzelnen maskulinen Kasusmarker feststellen, die offensichtlich, wie oben bereits erwähnt, beim Maskulinum nicht nur den relativen Anteil distinkter Einzelbelege im Diasystem, sondern zudem die geographische Verbreitung des Musters N/A/D im Untersuchungsraum spiegelt. Je größer die Ausdehnung des erhaltenen Distinktionsgebiets in der jeweiligen Wortart ist, desto kleiner ist das geographische Gebiet, in dem sich eines der synkretischen Muster (NA/D oder N/AD) durchgesetzt hat. Hierbei handelt es sich um eine Struktur, die ähnlich, wenn auch recht grob, bereits in Shrier (1965) beschrieben ist:

[. . .] is the southwestern territorial patterning of isoglosses (Map 13) which display the strength of the N/A/D system from the central, most conservative dialect regions to the peripheral, more dynamic regions.

(Shrier 1965: 434-435)

Shrier (1965: 434-435) beschreibt im Südwesten (etwa im Bereich des Untersuchungsgebiets der Korpusanalyse) ein Distinktionsgebiet, das im Osten durch 
ein Isoglossenbündel und im Westen durch eine Staffelung von Isoglossen, die sich auf die Muster maskuliner Wortarten beziehen, begrenzt ist. Auf Grundlage der Daten der Korpusanalyse ist eine solche Staffelung deutlich weiter östlich als in Abb. 7 nach Shrier (1965) angenommen und zusätzlich auch im Süden des Distinktionsgebiets anzusetzen. Zudem deutet sich hier an, dass keinesfalls von einer solch klaren Untergliederung durch Isoglossen, wie sie dort suggeriert ist, ausgegangen werden kann (vgl. dazu 4.3.3 und 4.3.4).

Shrier (1965: 435) weist an dieser Stelle jedoch noch auf einen zusätzlichen, für die Skala in (12) äußerst relevanten Aspekt hin: Mit Blick auf die Erkenntnisse zur diachronen Entwicklung des (ober)deutschen Kasussystems (vgl. 2.2.2.1) kann die Skala als geographische Ausdehnung eines synkretischen Musters analog zu den übrigen Kategorien der 3. Person auch in maskulinen Kategorien interpretiert werden, die sich kategoriell entlang der einzelnen Wortarten (angefangen beim Adjektiv und über Artikel und Demonstrativpronomen hin zum Personalpronomen) vollzieht und in einem letzten „konservativen“ Gebiet bei den zentralen Kasusmarkern noch nicht durchgeführt ist. Dabei decken die Daten der Korpusanalyse lediglich einen relativ kleinen Raum ab, der jedoch ein zentrales Gebiet der kasusmorphologischen Gliederung des Oberdeutschen offenbart hat: Das Kerngebiet der erhaltenen maskulinen Kasusdistinktion.

Um diese Beobachtungen zur geographischen Gliederung in einen größeren räumlichen Kontext zu setzen, wurden zu den Daten der Ortsgrammatiken analoge Abbildungen erstellt. Da es sich bei den Daten aus den Ortsgrammatiken nicht um Frequenzdaten handelt und die Muster damit an jedem Ortspunkt nur einmal belegt sind, markieren hier nicht in der Größe variierende Kuchendiagramme, sondern farbige Punktsymbole die Belege der einzelnen Muster. In den Fällen, in denen sich aus den Paradigmen Mustervariation rekonstruieren ließ, sind diese Punktsymbole in einheitlicher Größe mehrfarbig gestaltet. Die kleinen grauen Punktsymbole markieren hier wieder Orte, an denen aus den Daten für die entsprechende Wortart kein Muster der Kasusmarkierung rekonstruiert werden konnte. ${ }^{89}$

Die Ortspunkte, die auf Basis der Ortsgrammatiken analysiert werden konnten (vgl. Abb. 8), sind keinesfalls homogen über das Gebiet des oberdeutschen Raums verteilt. Für das Zentrum und im Südosten, d. h. für das südöstliche Württemberg, für Bayern sowie für Österreich liegen nur äußerst vereinzelt Ortsgram-

89 Die Darstellungen konzentrieren sich hier ausschließlich auf Nominativ, Akkusativ und Dativ. Genitivformen, die sich durchaus in den Ortsgrammatiken finden, werden in den Karten nicht aufgeführt. 
matiken vor. Wie bereits oben angemerkt, betrifft dieser weiße Fleck im Ortsnetz auch große Teile des Gebiets, auf dem die Korpusanalyse angestellt wurde. Während sich zwischen diesem und dem Netz der Ortsgrammatiken im Norden und Westen Überschneidungspunkte bieten, wird der Süden und Osten des Untersuchungsraums nicht von Daten der Ortsgrammatiken abgedeckt. Es sind daher nur in bestimmten Gebieten direkte Vergleiche mit den Korpusdaten möglich. Das Ortsnetz bietet jedoch einen guten Rahmen, um die geographische Raumgliederung für den oberdeutschen Raum um das Untersuchungsgebiet der Korpusanalyse zu analysieren sowie die Möglichkeit, unter Berücksichtigung der oben angesetzten Chronologisierung der Daten erste Hinweise auf diachrone Wandelprozesse in den räumlichen Strukturen zu eruieren.

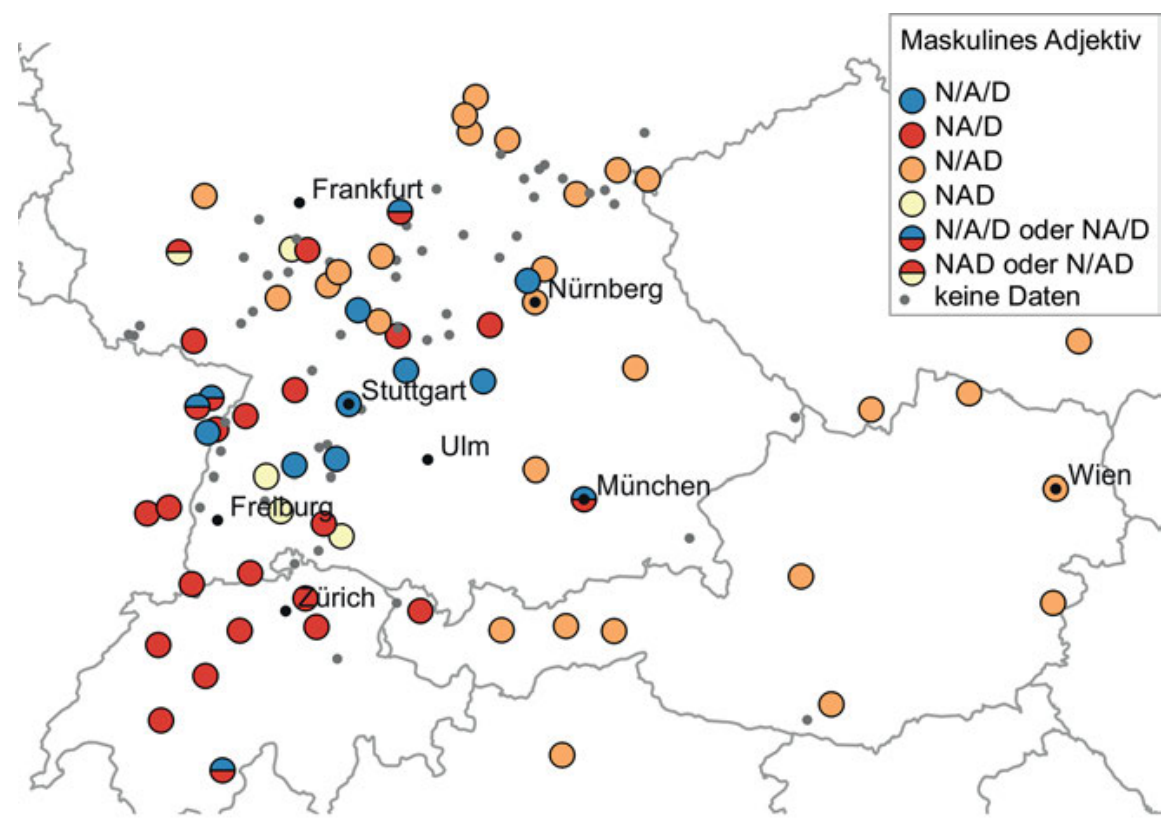

Abb. 32: Kasusmorphologische Raumbildung anhand der Daten aus Ortsgrammatiken zum maskulinen Adjektiv.

Abbildung 32 zeigt die Karte zu den Mustern der Kasusmarkierung beim maskulinen Adjektiv, wobei sich die Ortsgrammatiken größtenteils auf das Paradigma des starken Adjektivs konzentrieren. Ähnlich wie bei der Karte zu den Korpusdaten 
zeigt sich auch hier ein besonders hoher Anteil an verschiedenen synkretischen Mustern. Im zentralen und südlichen Westen des Raums dominiert das Muster NA/D; im Osten sowie im Norden des Gebiets tritt es nur vereinzelt auf. Hier dominiert das Muster N/AD. Belege für totalen Synkretismus NAD zeigen sich im zentralen und vereinzelt auch im nördlichen Westen. Die Karte unterschiedet sich besonders in einem Punkt von der zu den Korpusdaten in Abb. 27: Während sich dort ausschließlich synkretische Belege zeigen, finden sich in den Daten der Ortsgrammatiken auch Belege für volle Distinktion N/A/D, die insbesondere im Untersuchungsraum der Korpusanalyse auftreten. Diese Belege konzentrieren sich größtenteils im (westlichen) Zentrum des Gebiets als nahezu abgeschlossenes Areal, die Belege des Musters N/AD treten im Norden und im gesamten Osten auf und die des Musters NA/D im Südwesten und vereinzelt auch im Zentrum (außerdem als Einzelbeleg in Wien). An den Grenzen des zentralen Distinktionsraums zeigt sich in einzelnen Orten Variation zwischen der vollen Distinktion und dem Muster NA/D. Bei den Korpusdaten in Abb. 27 hat sich hingegen lediglich die Konzentration des totalen Synkretismus im südwestlichen Zentrum angedeutet, die sich jedoch auch in Abb. 32 zeigt. In den Daten der Ortsgrammatiken konnten also sogar beim maskulinen Adjektiv eindeutige raumgliedernde Strukturen herausgearbeitet werden.

Für diesen Unterschied zu den Daten der Korpusanalyse sind grundsätzlich zwei Erklärungsansätze denkbar: Zum einen können die Belege des Musters N/A/D durch das am Standarddeutschen bzw. an älteren Sprachstufen orientierte und damit distinkte Bezugssystem, das den Ortsgrammatiken häufig zugrunde liegt, beeinflusst sein. Ein großer Teil der Belege ist jedoch keineswegs zufällig über das Gebiet verteilt, sondern zeigt Raumbildung im Zentrum des oberdeutschen Gebiets und damit in dem bereits für zentrale Kasusmarker in der Korpusanalyse angesetzten Distinktionsraum. Daher wäre es zum anderen auch denkbar, dass der Unterschied durch einen (diachronen) Abbau der Kasusdistinktion beim Adjektiv - von noch im Kerngebiet vorhandener Distinktion zum durch die Ortsgrammatiken abgebildeten Sprachstand bis zur vollständigen Nivellierung im Korpus - zu erklären ist.

Der maskuline Indefinitartikel zeigt wie bereits in den Korpusdaten auch in den Ortsgrammatiken eine relativ klare Raumbildung (vgl. Abb. 33). Die Belege des distinkten Musters N/A/D befinden sich hier im Zentrum sowie im Norden, auch nördlich des Untersuchungsraums der Korpusanalyse, wo sie vereinzelt Variation mit dem Muster NA/D zeigen. Im gesamten Südwesten, in der Schweiz und im südlichen Baden, dominiert das Muster NA/D und bildet dort sogar einen homogenen Raum. Im Osten finden sich vereinzelt Belege für die volle Distinktion (etwa im Raum München). Ansonsten dominiert dort das Muster N/AD. 


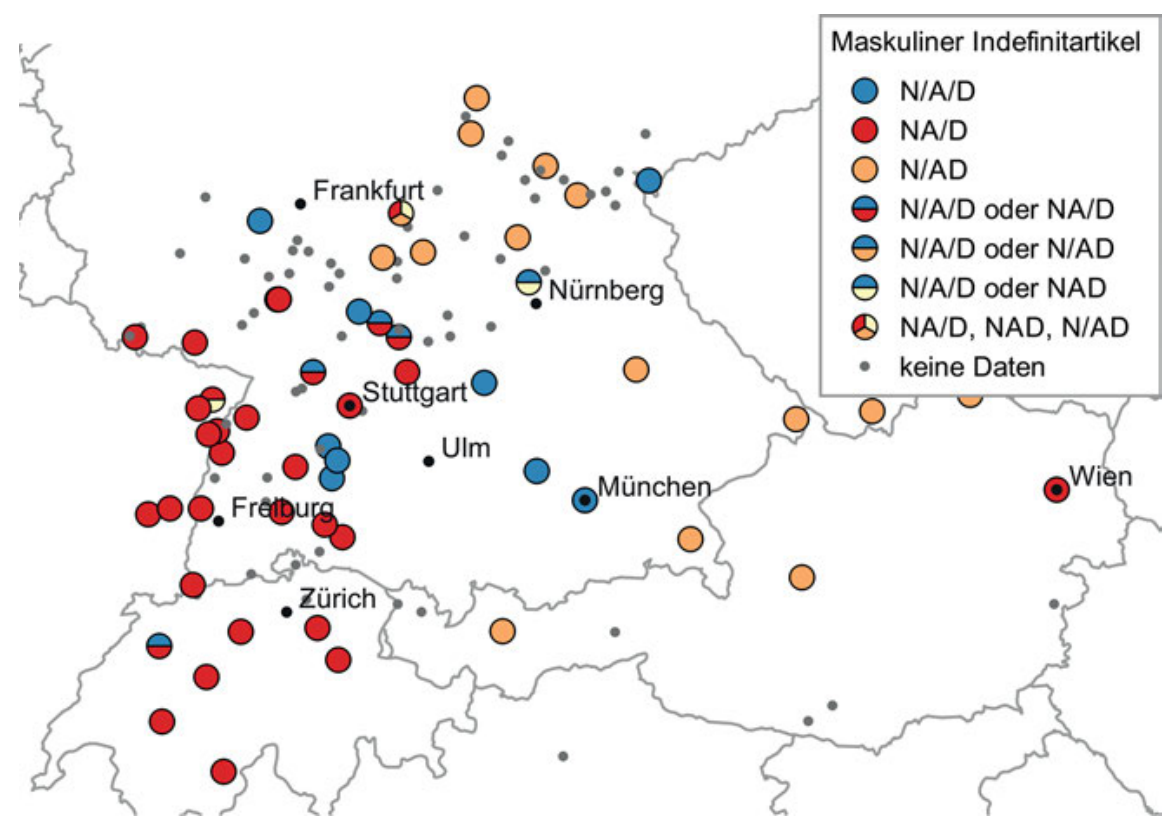

Abb. 33: Kasusmorphologische Raumbildung anhand der Daten aus Ortsgrammatiken zum maskulinen Indefinitartikel.

Auch hier nimmt das Distinktionsgebiet auf Grundlage der Ortsgrammatiken einen größeren Raum ein als auf der entsprechenden Karte zu den Korpusdaten. Insbesondere für die Muster im Kerngebiet könnte wieder ein diachroner Wandel als Erklärung herangezogen werden; für die vereinzelt auftretenden Belege fernab dieses Gebiets (z. B. im äußersten Nordosten) ist jedoch der Einfluss des standarddeutschen bzw. historischen Bezugssystems eine realistischere Erklärung.

Eine Wortart, für die sich aus den Korpusdaten keine vollständigen Muster rekonstruieren ließen, ist das Possessivum..$^{90}$ In den Ortsgrammatiken wird es hingegen häufig aufgeführt, sodass in Abb. 34 eine Karte zur geographischen Gliederung der Muster der Kasusmarkierung erstellt werden konnte. Verglichen mit dem Adjektiv und dem Indefinitartikel zeigen sich hier deutlich mehr Belege für das Muster der vollen Distinktion. Diese bilden einen halbwegs homogenen Raum in nördlichen Baden-Württemberg. Am nördlichen Rand dieses Raums sowie im Süd-

90 Unter diese Kategorie fallen in den Ortsgrammatiken teilweise Possessivpronomen und Possessivdeterminierer. Diese werden jedoch zumeist nicht getrennt aufgeführt. 
westen von Baden-Württemberg und in der gesamten Schweiz zeigt sich das Muster NA/D, das sich vereinzelt auch im Osten des Gebiets findet, wo ansonsten soweit durch das lose Ortsnetz ersichtlich - ebenso wie beim Indefinitartikel das Muster N/AD dominiert. Auch hier zeigt sich am nordöstlichen Rand des Distinktionsgebiets Mustervariation.

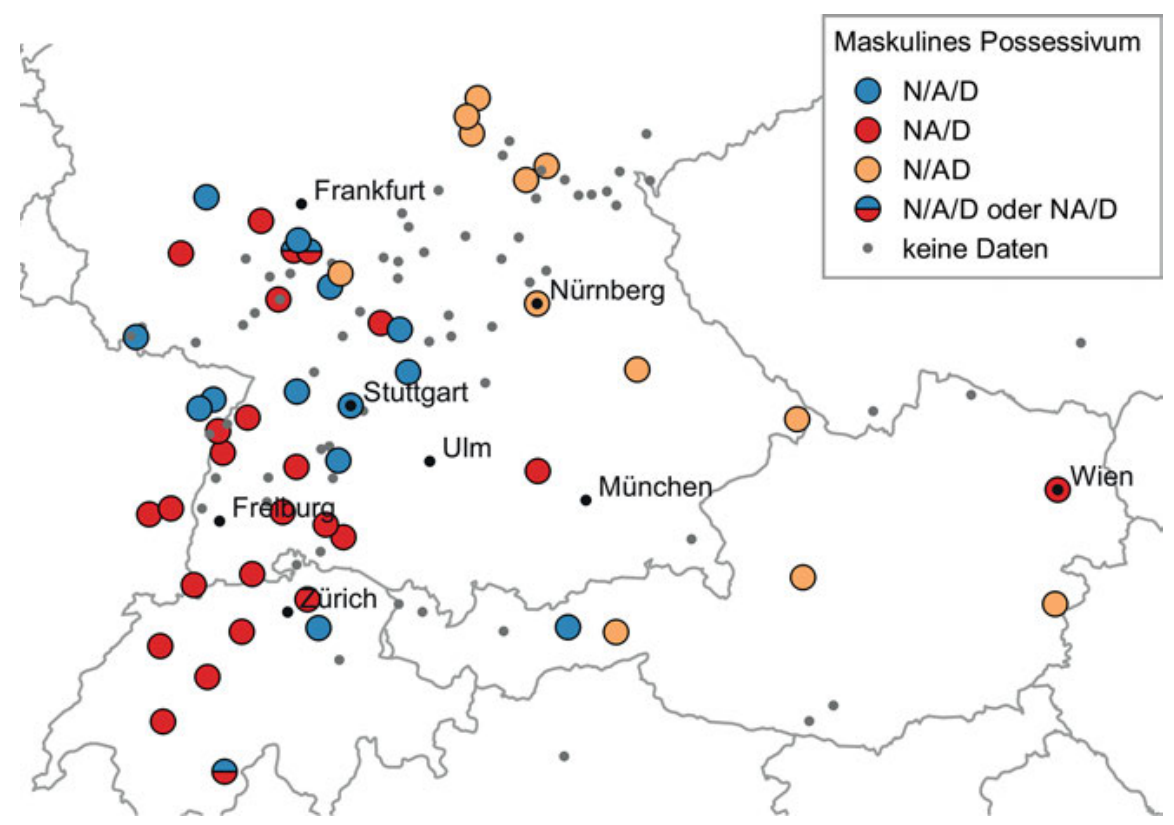

Abb. 34: Kasusmorphologische Raumbildung anhand der Daten aus Ortsgrammatiken zum maskulinen Possessivum.

Wie bereits die Korpusdaten bilden auch die Daten der Ortsgrammatiken ein außerordentlich dichtes Belegnetz beim maskulinen Definitartikel. Dies liegt jedoch - anders als in den Korpusdaten - nicht an der hohen Tokenfrequenz dieser Wortart, sondern v. a. daran, dass sich die Paradigmen des Definitartikels, soweit sie nicht ohnehin explizit in einer Grammatik aufgeführt sind, häufig aus anderen Beschreibungen beispielweise zur Nominalflexion rekonstruieren lassen. Auf diese Weise lässt sich auch hier ein äußerst scharfes Bild der geographischen Gliederung zeichnen.

Wie bereits bei den oben beschriebenen Wortarten findet sich das Muster NA/D auch in den Ortsgrammatiken durchgängig in der Schweiz sowie vereinzelt im nördlich davon gelegenen Distinktionsgebiet. Das Muster N/A/D dehnt sich scheinbar vom Zentrum weiter in den Norden und auch in den Osten bis 
nach München hin aus (aufgrund der wortartenübergreifenden Lücken im Ortsnetz muss diese Aussage etwas vorsichtig formuliert werden). Es zeigt zwar im Nordosten Variation mit dem Muster NA/D, bildet aber ansonsten besonders im Raum Stuttgart ein noch deutlich homogeneres Gebiet, als dies in den Korpusdaten der Fall ist. Auch hier zeigt der Osten ansonsten relativ homogen das Muster N/AD.

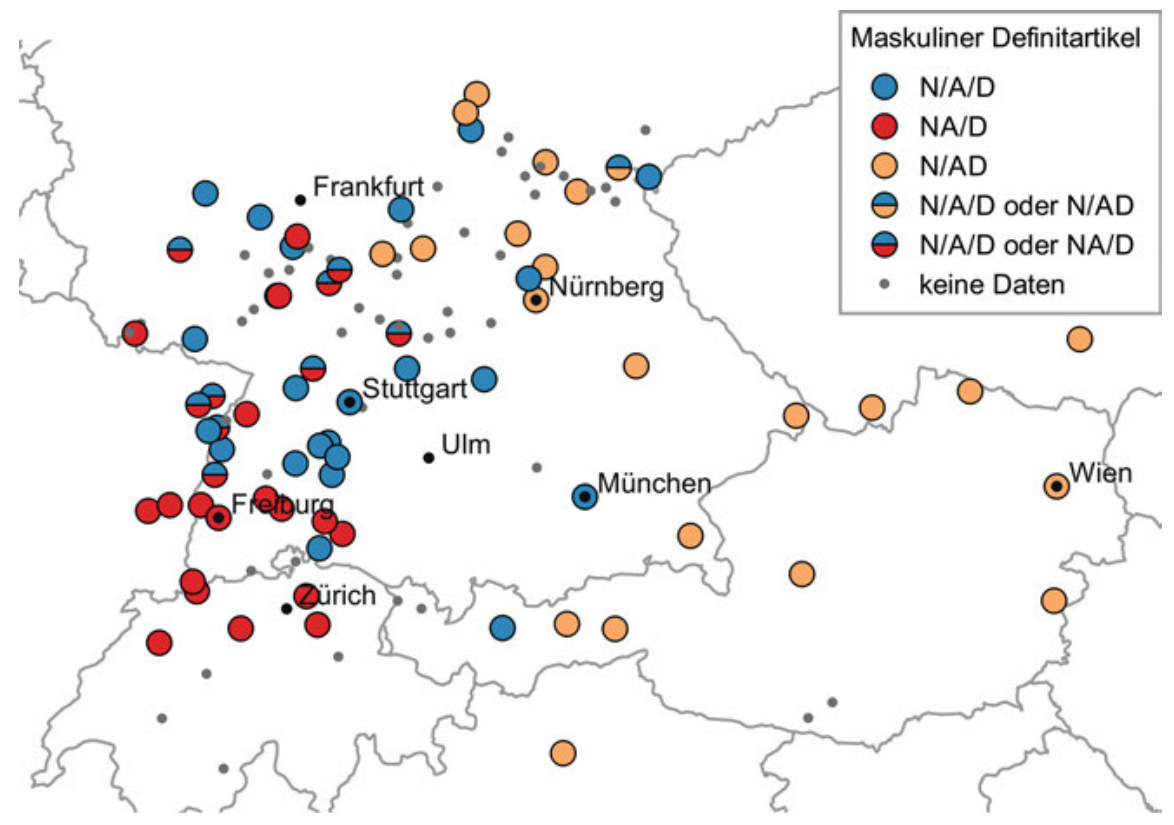

Abb. 35: Kasusmorphologische Raumbildung anhand der Daten aus Ortsgrammatiken zum maskulinen Definitartikel.

Wenn auch mit einem weniger dichten Belegnetz, zeigt das maskuline Demonstrativpronomen ebenfalls ein recht klares Raumbild. Das Gebiet mit dem Muster NA/D ist bis auf wenige Ausnahmen vollständig auf das südliche Baden, die Schweiz (wo sich lediglich ein abweichender Beleg für volle Distinktion zeigt) sowie auf das Elsass beschränkt. Die volle Distinktion (N/A/D) hat verglichen mit den bisher angeführten Wortarten ein äußerst großes Verbreitungsgebiet, das das gesamte Zentrum und den Norden von Baden-Württemberg, sowie die südlich noch analysierten Bereiche von Hessen und Rheinland-Pfalz umfasst und sich im Osten bis etwa auf die Höhe von München erstreckt. Das übrige östliche Gebiet weist auch hier das Muster N/AD auf. 
Da die Korpusanalyse kein flächendeckendes Bild des maskulinen Demonstrativpronomens gezeigt hat, ist kein direkter Vergleich mit diesen Erkenntnissen aus den Ortsgrammatiken möglich. Die großflächige Ausdehnung des Distinktionsgebiets, die sich in den Korpusdaten angedeutet hat, lässt sich jedoch auch in Abb. 36 erkennen.

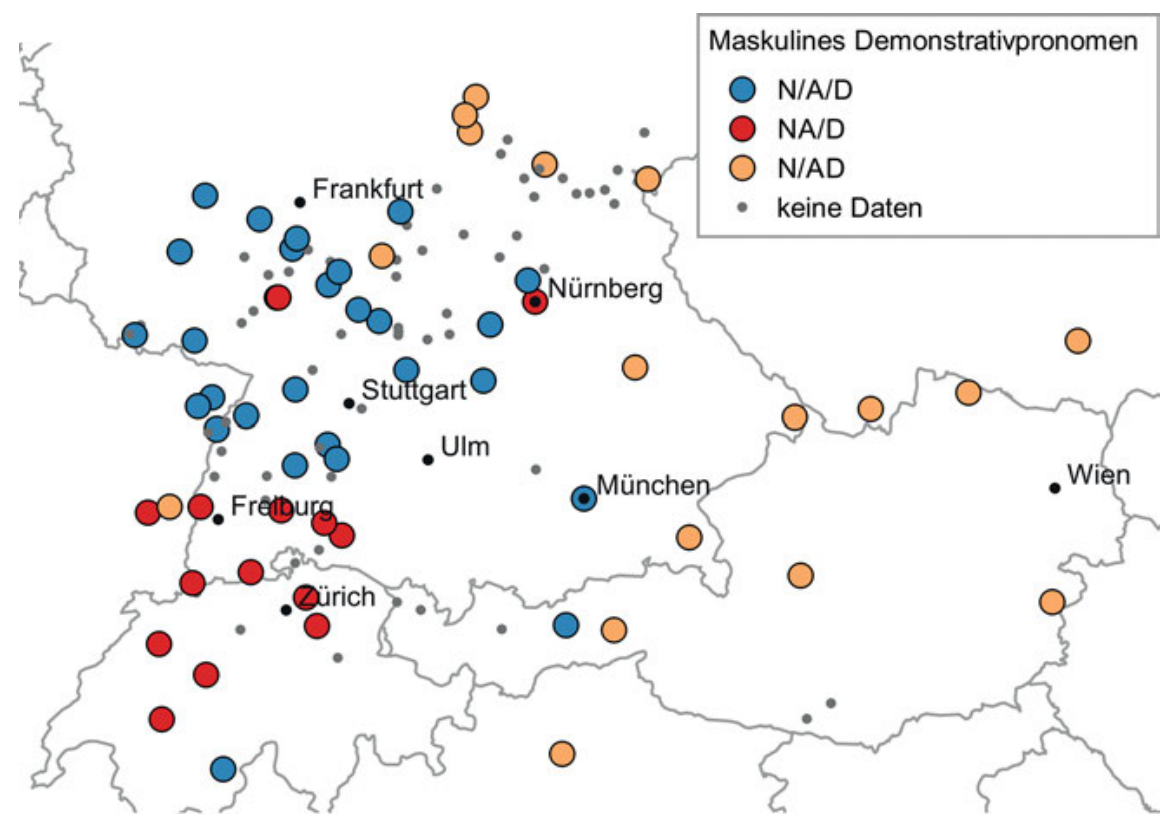

Abb. 36: Kasusmorphologische Raumbildung anhand der Daten aus Ortsgrammatiken zum maskulinen Demonstrativpronomen.

In Bezug auf das Personalpronomen zeigt sich auch in den Daten der Ortsgrammatiken eine deutliche Dominanz der Belege für das Muster der vollen Distinktion (vgl. Abb. 37). Hier ergibt sich für das oberdeutsche Gebiet verglichen mit den bisherigen Wortarten ein erstaunliches Bild: Das geschlossene Gebiet mit Muster NA/D im Westen und Südwesten ist nicht mehr zu erkennen. Stattdessen dominiert nahezu im gesamten westlichen Teil des Untersuchungsgebietes die volle Distinktion. Der Osten hingegen, der bislang recht einheitlich das Muster N/AD gezeigt hat, scheint, soweit auf Basis des lockeren Ortsnetzes erkennbar, im Südwesten bis nach Bad Aussee ebenso die volle Distinktion aufzuweisen, während sich im Südosten neben dem für den Osten im Maskulinum üblichen Muster N/AD auch ein Gebiet mit NA/D abzeichnet - ein synkretisches Muster, das sich auf diesem Gebiet ansonsten nicht im Maskulinum findet. Le- 
diglich im Nordosten zeigt sich ein relativ dicht belegtes Gebiet mit dem im Osten in anderen Wortarten so üblichen Muster N/AD.

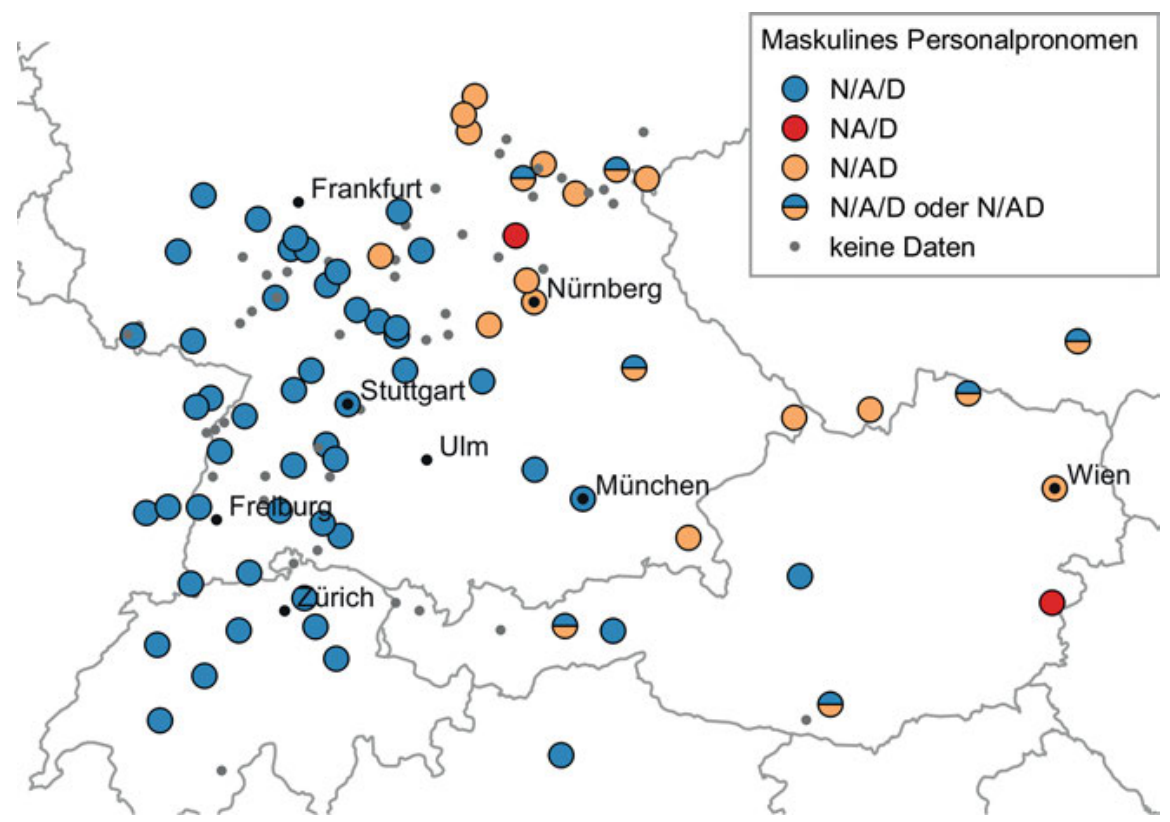

Abb. 37: Kasusmorphologische Raumbildung anhand der Daten aus Ortsgrammatiken zum maskulinen Personalpronomen.

Das Personalpronomen sticht damit nicht nur wegen seiner außerordentlichen Distinktionsquote von 99\% im Untersuchungsgebiet der Korpusanalyse gegenüber den übrigen maskulinen Kategorien hervor. Die Notwendigkeit einer distinkten Markierung des Personalpronomens im Kasusdiasystem scheint derart groß zu sein, dass nur ein beschränktes Gebiet des Oberdeutschen hier ein synkretisches Muster zulässt, wobei eine distinkte Nominativmarkierung (bis auf sehr wenige Ausnahmen) im gesamten oberdeutschen Raum gewährleistet ist.

Die Erkenntnisse zur kasusmorphologischen Raumgliederung anhand maskuliner Kategorien können wie folgt gebündelt werden: Basierend auf den Daten der Ortsgrammatiken lässt sich die Skala in (12) zur kategoriell gestaffelten geographischen Ausdehnung des Distinktionsgebiets in den Korpusdaten folgendermaßen verfeinern:

(13) Adjektiv < Indefinitartikel < Possessivum < Definitartikel < Demonstrativpronomen $<$ Personalpronomen 
Das Adjektiv hat zwar in den Daten der Ortsgrammatiken auch einen gewissen Anteil des Musters N/A/D gezeigt, dieser ist jedoch deutlich geringer als in den übrigen Wortarten. Das Possessivum, das auf Basis der Korpusdaten nicht untersucht werden konnte, wurde zwischen dem Indefinitartikel und dem Definitartikel verortet.

In den Daten der Ortsgrammatiken hat das Distinktionsgebiet jeweils eine größere geographische Verbreitung als in den Daten der Korpusanalyse. Zwar muss durchaus ein gewisser Einfluss des häufig standarddeutschen bzw. historischen und damit distinkten Bezugssystems in den Ortsgrammatiken berücksichtigt werden, ein Großteil der distinkten Belege bildet jedoch auch hier einen größtenteils homogenen Raum im (westlichen) Zentrum des oberdeutschen Gebiets. Eine Erklärung, die ausschließlich auf diesem methodischen Aspekt basiert, der sich dann übergreifend auf das gesamte Gebiet auswirken würde, greift hier daher zu kurz. Dass das distinkte Muster zu dem von den Ortsgrammatiken abgebildeten Sprachstand noch eine größere geographische Verbreitung gezeigt hat und zum Zeitpunkt der Tonaufnahmen zu Ruoff (1984) schon weiteren Nivellierungstendenzen unterworfen war, kann hier durchaus als realistische Erklärung in Betracht gezogen werden.

Ein weiterer Aspekt, der bislang noch nicht speziell besprochen wurde, ist die Mustervariation auf Wortartenebene. Diese zeigt sich sowohl in den Korpusdaten als auch in denen der Ortsgrammatiken und tritt häufig, aber nicht ausschließlich an den Rändern des Distinktionsgebiets auf. Sie ist auf den Karten zum Adjektiv, beim indefiniten sowie beim definiten Artikel in beiden Datengrundlagen und beim Possessivum und Personalpronomen vereinzelt in den Daten der Ortsgrammatiken zu beobachten. Hier konnten an einem Ort nicht nur unterschiedliche Muster für verschiedene Wortarten (z. B. ein anderes Muster beim maskulinen Definitartikel als beim maskulinen Personalpronomen), sondern auch innerhalb des Paradigmas einer einzigen maskulinen Wortart rekonstruiert werden.

Tab. 26: Paradigmatische Reihe des maskulinen Definitartikels mit Mustervariation auf Wortebene, rekonstruiert aus dem Transkript Isingen/Balingen.

\begin{tabular}{ll}
\hline & Singular \\
\hline Nominativ & de \\
\hline Akkusativ & de oder $(d) e n$ \\
\hline Dativ & dem \\
\hline
\end{tabular}


Ein Beispiel zeigt Tab. 26 zum maskulinen Definitartikel im Transkript von Isingen/Balingen. Dort konnten für den Akkusativ zwei Formen rekonstruiert werden, wobei eine der des Nominativs entspricht (NA/D), während die andere distinkt ist (N/A/D).

In spezifisch dialektgeographischen Arbeiten, die sich mit großflächigeren Raumstrukturen auseinandersetzen, wurde dieses Phänomen bislang nicht behandelt, wohl aber in solchen, die sich auf kleinräumigere Gebiete beziehen. Obwohl es beispielsweise Überschneidungen zwischen den hier genutzten Ortsgrammatiken und der dort genutzten Datengrundlage geben muss, wird das Phänomen in Shrier (1965) nicht erwähnt. In 4.3.4 werde ich noch einmal ausführlicher auf dieses Phänomen eingehen, das sich als charakteristisch für bestimmte maskuline Wortarten erwiesen hat.

In 4.3.1 wurde neben den maskulinen Kategorien auch die 1. Person Singular als mögliche raumbildende Kategorie angeführt. Während die raumbildende Funktion der maskulinen Wortarten Shrier (1965: Karte 13) ausführlich besprochen wird, wird diese für das Personalpronomen der 1. Person Singular in dort lediglich in der Legende erwähnt, jedoch ansonsten nicht weiter ausgeführt. Dabei ist anzunehmen, dass die Wortart hier nicht in die Karte integriert wurde, weil sie sich als raumbildend erwiesen hat, sondern lediglich deshalb, weil sie teilsystemübergreifend als letztes Refugium der vollen Distinktion abgebildet werden sollte. Da sich auch in dieser Kategorie jedoch eine gewisse Mustervariation im Korpus gezeigt hat, erfolgt an dieser Stelle abschließend ein kurzer Blick in die geographische Verbreitung der Muster der Kasusmarkierung des Personalpronomens der 1. Person Singular.

Abbildung 38 zeigt, wie grundsätzlich auf Grundlage der Forschungsliteratur sowie der außerordentlich hohen Distinktionsquote von 99\% zu vermuten, einen besonders hohen Anteil an voller Distinktion in den Korpusdaten. Die Karte zeigt allerdings auch, dass die 94 Belege für das Muster N/AD (z. B. i/ me, me, Hettlingen/Wertingen_1) nicht willkürlich im Gebiet verbreitet, sondern an zwei nebeneinanderliegenden Orten am Ostrand des Untersuchungsraums, nämlich Achsheim/Augsburg und Hettlingen/Wertingen, ${ }^{91}$ konzentriert sind. Wie bereits in 4.3.1 gemutmaßt, könnte das Muster N/AD im Singular des Personalpronomens der 1. Person hier auf ein Übergreifen des für die 1. Person üblichen Synkretismusmusters vom Plural auf den Singular hindeuten. Interessant

91 Aus dem Ort Hettlingen/Wertingen liegen im Korpus zwei Transkripte vor. Alle Belege der 1. Person aus diesem Ort sind allerdings im Transkript Hettlingen/Wertingen_1 belegt. Im Transkript Hettlingen/Wertingen_2 konnte kein Muster für das Personalpronomen der 1. Person Singular rekonstruiert werden, was eine vergleichende Analyse - die gerade in Bezug auf dieses Muster interessant gewesen wäre - leider nicht ermöglicht. 
ist hierbei die geographische Lage dieser Belege am Ostrand des Untersuchungsgebiets. Auch in maskulinen Kategorien dominiert dort das Muster N/AD, was eine Ausbreitung des Musters auch im Singular der 1. Person von Osten aus durchaus nachvollziehbar machen würde. Die maskulinen Kategorien könnten damit als Katalysator für die Ausweitung des Pluralmusters der 1. Person auf Singularkontexte fungieren - eine These, die sich jedoch aufgrund der recht geringen Belegdichte und des kleinen Untersuchungsgebiets nicht empirisch untermauern lässt.

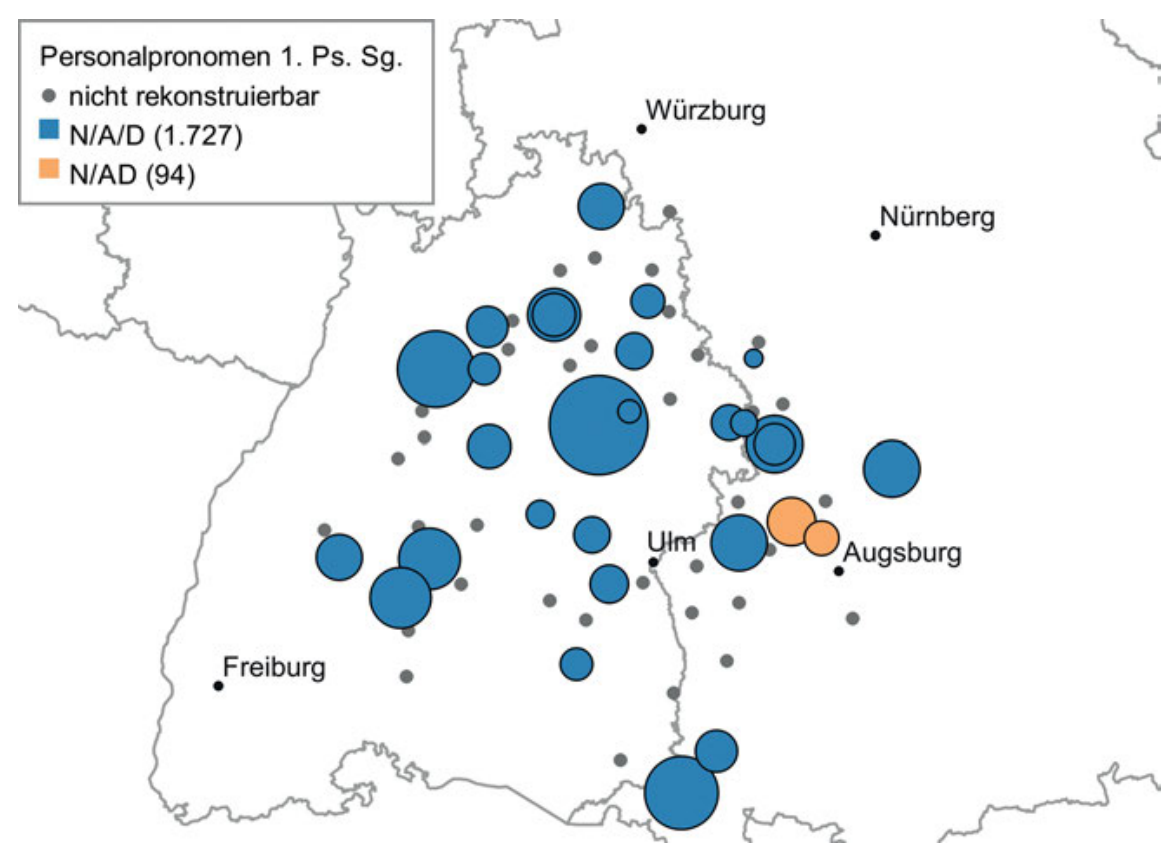

Abb. 38: Kasusmorphologische Raumbildung anhand der Korpusdaten zum Personalpronomen der 1. Ps. Sg.

Die Darstellung der Ergebnisse aus der Analyse der Ortsgrammatiken spricht eher dagegen, ein sich ausbreitendes Muster N/AD beim Personalpronomen der 1. Person Singular anzusetzen (vgl. Abb. 39). Zwar fallen die beiden Orte, die in Abb. 38 das Muster N/AD gezeigt haben, in den Bereich, der nicht durch das Ortsnetz der Grammatiken abgedeckt ist, doch sind im gesamten oberdeutschen 


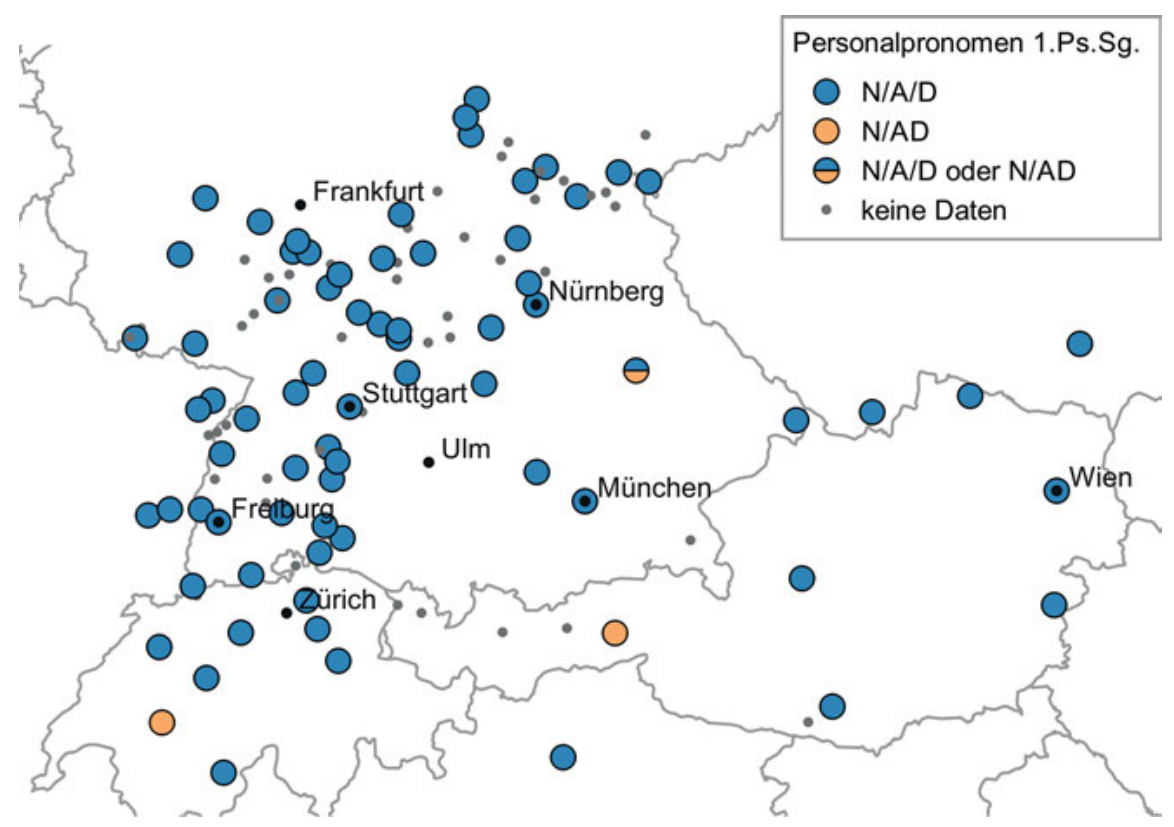

Abb. 39: Kasusmorphologische Raumbildung anhand der Daten aus Ortsgrammatiken zum Personalpronomen der 1. Ps. Sg.

Gebiet nur marginal Belege für dieses Muster zu erkennen, die zudem über den gesamten Raum verteilt sind. ${ }^{92}$

Wie bereits oben ausgeführt, ist davon auszugehen, dass die Daten der Ortsgrammatiken einen eher älteren Sprachstand abbilden als die der Korpusanalyse. Es ist daher durchaus denkbar, dass sich das Verbreitungsgebiet des Musters N/AD beim Personalpronomen der 1. Person Singular im Zeitraum zwischen den beiden in den verschiedenen Datengrundlagen abgebildeten Sprachständen verdichtet beziehungsweise geographisch ausgedehnt hat. An den wenigen und - bezogen auf die Korpusanalyse - geographisch zudem eher kleinräumigen Daten zu diesem Phänomen lässt sich eine raumbildende geographische Ausdehnung des Musters jedoch nicht nachweisen. Auf Basis dieser Analyse können damit weiterhin lediglich die maskulinen Kategorien als raumbildende Faktoren im oberdeutschen Gebiet angesetzt werden.

92 Auch Rabanus (2008: 254-255) beschreibt dieses Muster lediglich auf Grundlage von Funk (1957) für den Ort Ingertsheim (in Oberbayern), der weitab des Untersuchungsgebiets der Korpusanalyse liegt. 


\subsubsection{Erkenntnisse zu kasusmorphologischen Raumbildern}

Die Beschreibung der geographischen Gliederung der Muster anhand der einzelnen maskulinen Kasusmarker in 4.3.2 wird in diesem Teilkapitel als Grundlage genutzt, um übergreifende Regularitäten der kasusmorphologischen Raumgliederung herauszuarbeiten. Bereits in 2.3.3 wurde beispielsweise die Darstellung der Raumstruktur in Shrier (1965) und in Rowley (2004) kritisiert, die sich größtenteils an der Tradition der eher phonologisch-lexikalischen Dialektkartographie orientiert und homogene, durch Isoglossen separierte Raumstrukturen abbildet - ein Konzept, das selbst für phonologisch-lexikalische Phänomene inzwischen hinterfragt wird (vgl. dazu beispielsweise Pröll 2015). Bereits in Shriers (1965) Karten lassen sich erste Hinweise darauf erkennen, dass die geographische Gliederung auf Basis der Muster der Kasusmarkierung eher Raumbildern zu syntaktischen als solchen zu phonologisch-lexikalischen Phänomenen ähnelt - wie etwa die eher großflächige Raumbildung sowie die nach Wortarten gestaffelten Gebietsausdehnungen. Im folgenden Teilkapitel werde ich die kasusmorphologischen Raumbilder daher aufbauend auf den Eigenschaften, die sich in den Karten in 4.3.2 gezeigt haben, und angelehnt an bisherige Erkenntnisse zur morphosyntaktischen Raumgliederung beschreiben. Dabei zeigen sich jedoch auch einige Aspekte, in denen die kasusmorphologische Raumgliederung strukturell von der syntaktischen abweicht. Auf diese Besonderheiten werde ich abschließend noch einmal explizit eingehen.

Phänomene der Kasusmorphologie vor dem Hintergrund der morphosyntaktischen Raumgliederung zu beschreiben ist keine vollkommen neue Idee. Bereits in ihrer Übersicht zum Kernbereich geographischer morphosyntaktischer Variation führt Glaser (2017: 169) verschiedene kasusmorphologische Einzelphänomene auf, so z. B. die präpositionale Dativmarkierung, Possessivkonstruktionen sowie den Synkretismus von Akkusativ mit Nominativ und Dativ. In diesem Kapitel werde ich jedoch nicht nur einzelne das Kasussystem betreffende Phänomene, sondern im Rahmen der strukturell-dialektologischen Ausrichtung der Analyse vollständige Muster der Kasusmarkierung in ihrer geographischen Gliederung in den Blick nehmen und mit den Erkenntnissen zur morphosyntaktischen Raumstruktur in Bezug setzen.

Um die in 4.3.2 aufgeführten Einzelkarten und die dafür jeweils beschriebenen Beobachtungen zu einem Gesamtbild der kasusmorphologischen Raumbildung zu verknüpfen, wurde eine hierarchische Clusteranalyse auf Grundlage von Mustern der Kasusmarkierung in den maskulinen Wortarten durchgeführt. Die Datengrundlage wurde hierbei auf die Ortsgrammatiken beschränkt, da diese im Gegensatz zu den Korpusdaten ein äußerst homogenes Belegnetz 
aufweisen..$^{93}$ Das Belegnetz der Korpusdaten hat sich aufgrund der unterschiedlichen Ausschnitte des idiolektalen Systems, die in den einzelnen Transkripten wiedergegeben sind, als äußerst heterogen erwiesen, was eine geostatistische Analyse dieser Daten leider ausschließt.

Zunächst wurde ein hierarchisches Ward-Cluster ermittelt, das die Quadrierung der euklidischen Distanz zwischen zwei in den Ortsgrammatiken beschriebenen Belegorten wiedergibt (vgl. die prägnante Beschreibung der hierarchischen Clusteranalyse sowie den R-Code, der hier in leicht abgewandelter Form hier genutzt wurde, in Schäfer 2017: 313-314). ${ }^{94}$

Abbildung 40 zeigt das Dendrogramm, eine Baumdiagrammdarstellung der einzelnen Cluster, in die sich die einzelnen Ortspunkte (auf die sich die Ortsgrammatiken beziehen), anhand der dort rekonstruierten Muster der Kasusmarkierung gliedern lassen. Die Nummerierung der einzelnen Ortsgrammatiken, die sich jeweils am Ende der Zweige eines Clusters befinden, ist wieder über das Verzeichnis in Anhang 6.1 aufgeschlüsselt. Das Dendrogramm ist dabei wie folgt zu verstehen: Die Ortspunkte, die auf einer niedrigeren Stufe des Dendrogramms miteinander verbunden sind - die eher feine Verästelungen zeigen - sind einander ähnlicher als solche, die auf einer höheren Stufe miteinander verbunden sind - also durch eher dicke Astgabeln verbunden sind.

Die einzelnen Ortspunkte wurden basierend auf ihrer Zugehörigkeit zu dem jeweiligen Cluster eingefärbt und in Abb. 41 kartiert. Die Farben der einzelnen Cluster wurden zufällig ausgewählt.

Der oberdeutsche Raum lässt sich auf diese Weise wie folgt gliedern: Es können ganz grundlegend drei größere Gebiete in Abb. 41 angesetzt werden. Der gesamte Osten des Oberdeutschen (vgl. Cluster 3) scheint hier (dies muss aufgrund des grade dort sehr losen Ortsnetzes vorsichtig formuliert werden) bei allen maskulinen Wortarten (mit Ausnahme des Personalpronomens vollständig) zum Muster N/AD zu tendieren - ein Muster, das für die 3. Person eher untypisch ist und eher in der 1. Person (insbesondere im Plural) dominiert (vgl. 4.2.3).

93 Zur Differenzierung von Orts- und Belegnetz s. 3.2.2.

94 Im Folgenden genutztes, überarbeitetes R-Skript nach Schäfer (2017: 313):

$\mathrm{d}<-\operatorname{dist}($ DATEINAME, method =“euclidean")

\#für Distanzmatrix

fit <- hclust(d, method=“ward.D2")

plot(fit, hang=-1)

\#erstellt ein Dendrogramm

groups <- cutree(fit, k=5) ->Baum

\#teilt den Baum in 5 Cluster

rect.hclust(fit, $\mathrm{k}=5$, border="grey")

\#zeichnet graue Quadrate um die 5 Cluster 

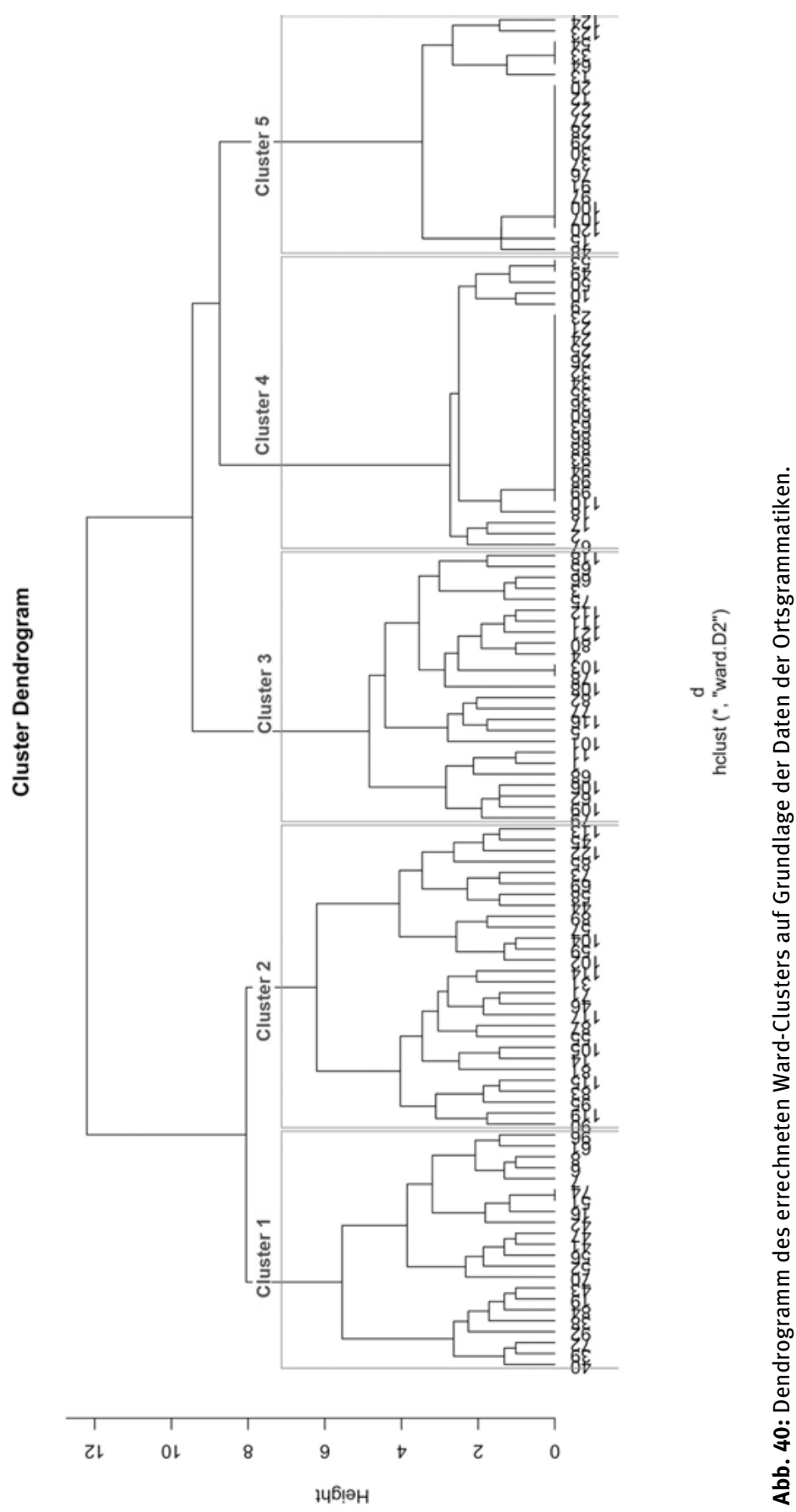


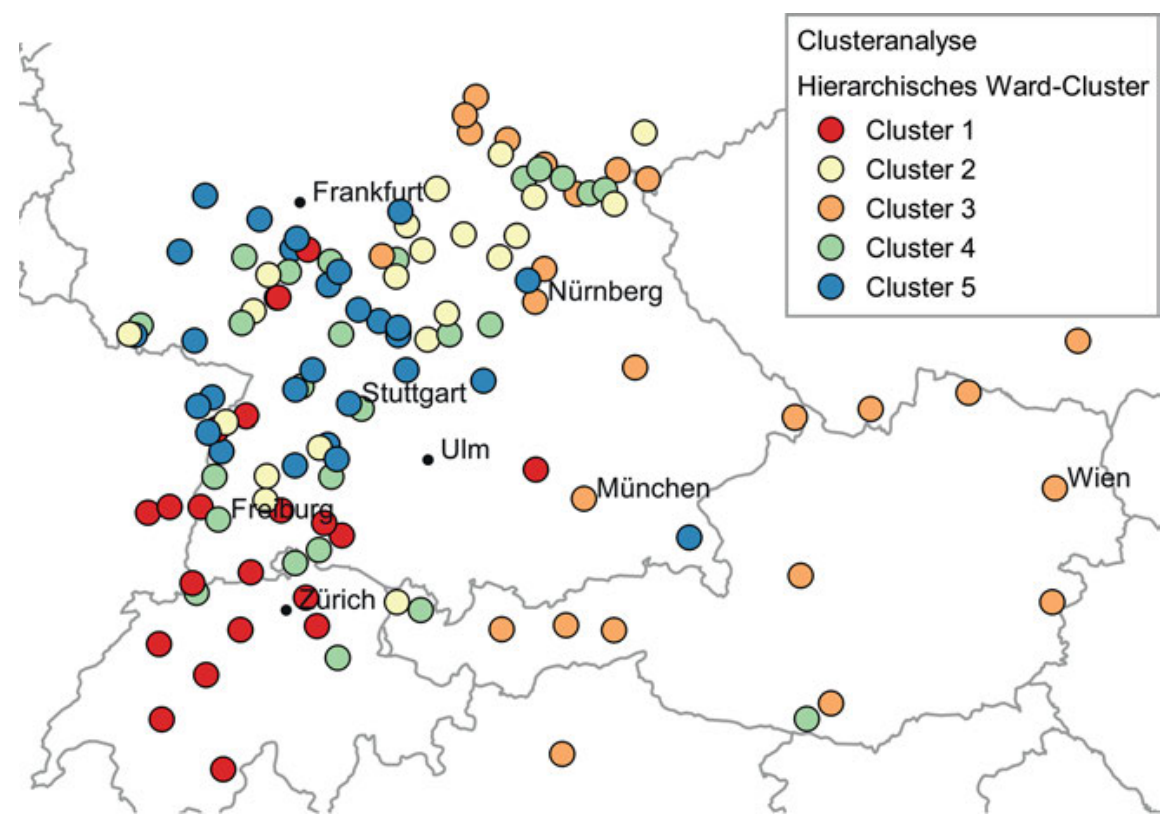

Abb. 41: Kasusmorphologische Raumbildung anhand einer Clusteranalyse auf Grundlage aller maskulinen Kasusmarker.

Dieses Muster ist für das oberdeutsche Gebiet insofern bemerkenswert, als es die ansonsten hier so prominente Dativdistinktion betrifft. Dal (1971a) vermutet diesbezüglich ein eher phonologisch als morphologisch determiniertes Muster, das keine „syntaktische Einebnung der beiden Kasus [Akkusativ und Dativ] überhaupt“ zur Folge hat (Dal 1971a: 188). Aus Perspektive der Systemfunktion, die in dieser Arbeit auf Grundlage der morphophonologischen Oberflächenstruktur herausgearbeiteten wurde, wird zwar dennoch Akkusativ-Dativ-Synkretismus angesetzt, im Folgenden wird sich aber zeigen, dass sich der Raum mit Muster N/AD auch dialektgeographisch anders verhält als die beiden anderen, eher durch vermutlich innermorphologisch determinierte Muster geprägten Räume.

Der gesamte Westen lässt sich grob in zwei weitere Gebiete unterteilen. Im Südwesten, vornehmlich in der Schweiz sowie im südlichen Baden, findet sich bei nahezu allen maskulinen Wortarten - mit Ausnahme des Personalpronomens relativ homogen das Muster NA/D (vgl. Cluster 1). Das Maskulinum ist den übrigen Genera sowie dem Plural der 3. Person im Muster der Kasusmarkierung nahezu vollständig angepasst. Lediglich das suppletive Personalpronomen trägt hier noch die letzten Reste einer Nominativ-Akkusativ-Distinktion. Im Gegensatz zum Sonderstatus, den das Maskulinum im östlichen Gebiet mit dem ansonsten 
in der 3. Person unüblichen Muster N/AD einnimmt, kann die Ausbreitung des Musters NA/D ins Maskulinum, die in diesem Raum zu erkennen ist, als letzte Konsequenz einer bereits in 2.2.2.1 beschriebenen eher morphologisch determinierten diachronen Entwicklung gewertet werden, in der ein Muster der Kasusmarkierung für die 3. Person etabliert und ausgebaut worden ist.

Der dritte Raum, der sich vom Norden bis zum Zentrum des westlichen Gebiets erstreckt, weist deutlich mehr Heterogenität auf als die beiden bisher beschriebenen Räume (vgl. Cluster 2, 4 und 5 sowie einen Großteil der Daten der Korpusanalyse in 4.3.2, die nicht in die Clusteranalyse integriert werden konnten). Hier tritt die in 4.3.2 ausführlich beschriebene geographische Staffelung des Musters NA/D zur vollen Distinktion gegliedert nach den einzelnen maskulinen Wortarten zutage: Bis hin zum Kerngebiet der vollen Distinktion, das sich an der östlichen Grenze des westlichen Raums - etwa an der politischen Grenze zwischen Baden-Württemberg und Bayerisch-Schwaben - befindet, lässt sich hier eine zunehmende geographische Ausbreitung des Musters NA/D gestaffelt nach den einzelnen zentralen maskulinen Kasusmarkern beobachten. Mit Blick auf die angesetzte Chronologisierung der Daten und die weitere Ausdehnung des Musters NA/D in den Korpusdaten kann man hier einen Raum vermuten, in dem der Prozess der Nivellierung der Nominativ-Akkusativ-Distinktion, der im Südwesten bereits abgeschlossen ist, noch im Gange ist.

Die Raumgliederung des Oberdeutschen kann mit lediglich drei unterschiedlichen Teilsystemen durchaus als großflächige Struktur beschrieben werden und zeigt damit bereits ein erstes Charakteristikum der morphosyntaktischen Raumgliederung (vgl. beispielsweise Glaser 2008: 101, Glaser 2017: 175). Die charakteristische Ost-West-Gliederung morphosyntaktischer Phänomene, die Glaser (2017: 174) konkret auf die oben angeführten synkretischen Muster der Kasusmarkierung bezieht, lässt sich (wenn auch nicht ausschließlich, doch zumindest grundlegend) hier nur für ein begrenztes Gebiet bestätigen. Im Süden des oberdeutschen Gebiets zeigen die Ortsgrammatiken tatsächlich eine klare Unterteilung des Musters NA/D im alemannischen Raum, d.h. in der Schweiz und in Vorarlberg, und des Musters N/AD in Österreich. Diese klare Differenzierung wird lediglich beim Personalpronomen (wo die volle Distinktion im alemannischen Bereich das Muster N/AD ablöst) sowie durch einzelne Belege der vollen Distinktion im Übergangsbereich unterbrochen.

Nördlich davon, auf bundesdeutschem Gebiet, lässt sich eine solche OstWest-Gliederung nicht erkennen. Zwar finden sich hier prinzipiell Unterschiede zwischen dem östlichen Gebiet, in dem N/AD dominiert, und dem westlichen Raum, in dem NA/D und N/A/D interagieren, doch lassen sich die Grenzen beziehungsweise Ausdehnungen dieses Gebiets nicht derart klar bestimmen. So nimmt beispielsweise das Muster N/AD etwa beim Adjektiv und Indefinitartikel 
nahezu den gesamten Norden ein. Auch innerhalb des westlichen Raums kann weder von einer Ost-West-Gliederung noch von der zweiten in Glaser (2017: 174) als charakteristisch angeführten Struktur, der Nord-Süd-Gliederung, ausgegangen werden. Hier legen insbesondere die feinkörnigen Karten der Korpusdaten nahe, dass es sich bei der Staffelung des Distinktionsraums eher um ein Gebiet handelt, das sich ausgehend vom Kerngebiet der Distinktion nach Norden, Westen und Süden hin in Wellen ausdehnt. Die Gliederung der kasusmorphologischen Gebiete auf Grundlage vollständiger Muster der Kasusmarkierung scheint sich hier demnach zwar ähnlich, aber doch deutlich komplexer zu gestalten, als für zahlreiche andere morphosyntaktische (Einzel-)Phänomene angenommen wird. Dabei müssen diese feinen Unterschiede in der Raumgliederung nicht unbedingt auf spezielle Eigenschaften der Kategorie Kasus zurückzuführen sein. Es ist auch denkbar, dass durch die besonders feinkörnige Analyse gesprochensprachlicher Daten detailliertere Raumstrukturen aufgedeckt werden konnten als dies bei anderen Methoden und Datengrundlagen (etwa bei indirekten Erhebungen) der Fall ist.

Große Gemeinsamkeiten mit den bisherigen Erkenntnissen zur morphosyntaktischen Raumgliederung lassen sich in den Daten grundsätzlich bezüglich der Übergangsmuster zwischen den einzelnen oben angeführten Räumen beobachten, wobei zwei verschiedene Strukturen zu erkennen sind. Die Karten zeigen im Westen die charakteristische Übergangsstruktur einer schiefen Ebene wie sie in Seiler (2005) ${ }^{95}$ definiert ist: Das oberdeutsche Gebiet - insbesondere der zentrale Raum im Westen - weist kaum vollständig homogene Räume auf, sondern eher solche, in denen mehrere Muster der Kasusmarkierung parallel auftreten können - die also einen gewissen Anteil an Variation zeigen (vgl. Glaser 2017: 176-177). Übergänge zwischen diesen Gebieten äußern sich nun in der graduell ansteigenden Prominenz des einen und der analog abfallenden des jeweils anderen Musters (vgl. Seiler 2005: 330). Diese steigende bzw. fallende Prominenz lässt sich mit Seiler (2005: 331-332) grundsätzlich anhand von vier Charakteristika greifen: Der geographischen Belegdichte sowie der anteiligen Häufigkeit der Belege eines Musters je Ort, der Präferenz des Musters gegenüber anderen Varianten sowie der Beschränkung durch syntaktische Restriktionen.

95 Im Rahmen der kasusmorphologischen Raumgliederung wird die schiefe Ebene ausschließlich anhand von Seilers (2005) Charakterisierung genutzt. Das Konzept einer schiefen Ebene wird jedoch bereits bei Schmidt (1872: 27) als bildhafte Darstellung der Entstehung verschiedener indogermanischer Sprachen aus einem einzelnen kontinuierlichen Varietätenspektrum eingeführt. 


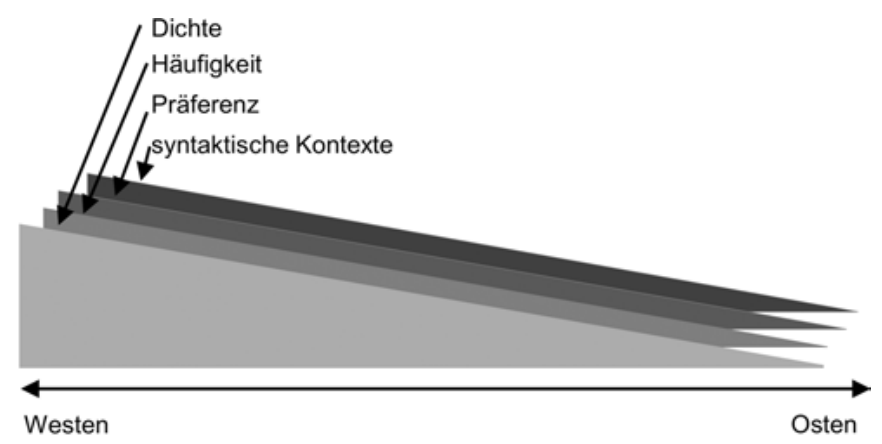

Abb. 42: Visualisierung des Konzepts der schiefen Ebene nach Seiler (2005: 332).

Die Präferenz bestimmter Muster lässt sich im Rahmen dieser Analyse nicht ermitteln - inwiefern eine Gewährsperson ein Muster präferiert bzw. als natürlicher empfindet, kann weder auf Basis von Ortsgrammatiken noch von Korpusdaten ausgemacht werden. Seilers (2005) Konzept fußt auf Daten der indirekten Erhebung zum Syntaktischen Atlas der deutschen Schweiz (SADS), die eben diese Präferenz im Fragebogen gezielt erhoben hat (vgl. beispielsweise Glaser 2000, Seiler 2005).

Die übrigen Charakteristika lassen sich jedoch auch anhand der hier genutzten Datenquellen untersuchen: Die geographische Belegdichte, d. h. die Menge der Orte, an denen sich ein Muster zeigt, ist sowohl in den Korpusdaten als auch in denen der Ortsgrammatiken zu erkennen und bereits oben beschreiben: So dominiert beispielsweise das Muster NA/D im nahezu vollständig homogenen südwestlichen Gebiet. Im zentralen Westen nimmt diese Dominanz zunehmend ab; immer mehr Belege der vollen Distinktion treten auf, bis diese im Kerngebiet der Distinktion schließlich dominieren. Auch die anteilige Häufigkeit der Muster lässt sich in den Korpusdaten ablesen: Die Anteile des Musters NA/D nehmen zum Distinktionsgebiet hin ab (vgl. 4.3.4). Insbesondere im zentral westlichen Gebiet, in dem teilweise starke Mustervariation auftritt, sind auch syntaktische Restriktionen für das Auftreten der einzelnen Muster denkbar. So lässt sich angelehnt an die Beschreibung Seilers (2005: 331) annehmen, dass das Muster NA/D im Südwesten immer auftritt, dass es im zentralen Gebiet bestimmten Restriktionen unterliegt und im Kerngebiet der Distinktion unterbleibt. Restriktionen dieser Art stellen zunächst grundlegend die einzelnen maskulinen Wortarten dar, in denen NA/D auftritt. Während dies im Südosten des Gebiets alle Wortarten außer dem Personalpronomen betrifft, findet sich das Muster nach Nordosten hin bei immer weniger Wortarten. In diesen Bereich des Übergangs fallen auch die meisten Belege der Mustervaria- 
tion auf Ebene einzelner Wortarten, die im westlichen Teil zwischen voller Distinktion und dem Muster NA/D variieren.

Im Osten des Gebiets - am Übergang zum Muster N/AD - zeichnet sich hingegen ein anderer Typus der morphosyntaktischen Übergangsstruktur ab: Auch hier tritt Mustervariation - sowohl solche, zwischen den verschiedenen maskulinen Wortarten als auch solche, auf Ebene einer einzelnen Wortart - als Übergangsstruktur auf, jedoch scheint diese geographisch deutlich begrenzter zu sein. Zwar ist die Belegdichte im östlichen Gebiet bei der Analyse der Ortsgrammatiken deutlich geringer als in den westlichen Gebieten, auf die auch der Untersuchungsraum der Korpusanalyse einen stärkeren Fokus legt, dennoch lässt sich relativ klar erkennen, dass sich die östliche Übergangsstruktur klar von der westlichen unterscheidet.

Dort lässt sich eher von der Struktur einer Stufe, wie sie in Leser (2012) beschrieben wird (vgl. Abb. 43), als von einer schiefen Ebene ausgehen. Eine solche Stufe zeigt nach Leser (2012: 97) einen deutlich abrupteren, räumlich deutlich begrenzteren Übergang als die schiefe Ebene an, grenzt sich durch auf beiden Seiten auftretende Variation jedoch vom Isoglossenkonzept ab. Auf beiden Seiten der Stufe können demnach durchaus noch Belege für das jeweils weniger prominente Muster gefunden werden, die Übergangszone nimmt jedoch einen deutlich geringeren geographischen Raum ein als dies bei der schiefen Ebene der Fall ist.

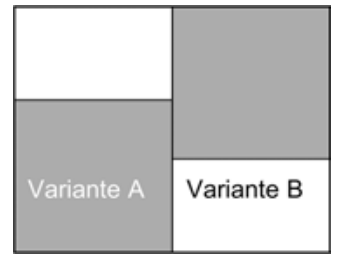

Abb. 43: Abbildung des Konzepts der Stufe nach Leser (2012: 97).

Auf Grundlage der Karten in 4.3.2 lässt sich dieser Stufenstruktur für die kasusmorphologische Raumgliederung noch ein weiteres Merkmal hinzufügen: Die Staffelung des synkretischen Musters nach maskulinen Wortarten, die die schiefe Ebene im Westen des Gebiets charakterisiert, tritt im Osten scheinbar nicht in diesem Umfang auf. Zwar unterschiedet sich die exakte Ausdehnung des N/AD-Gebiets insbesondere im Norden zwischen Adjektiv und Indefinitartikel einerseits und den übrigen Wortarten andererseits, doch scheint der Übergang zwischen dem östlichen und dem westlichen Gebiet ansonsten bei allen Wortarten nahezu parallel zu verlaufen (ganz ähnlich, wie bereits in den Karten in Shrier 1965 abgebildet). 
Die folgende Abbildung illustriert eine grobe Abstraktion des hier beschriebenen Übergangsszenarios entlang einer Achse, die quer durch den gesamten oberdeutschen Raum verläuft und Schnittpunkte mit allen drei oben beschriebenen Großräumen aufweist. Aufgrund der geographisch-strukturellen Ähnlichkeit orientiert sich Abb. 44 auch bezüglich der Darstellung der Stufenstruktur an der Darstellung der schiefen Ebene aus Seiler (2005: 332) in Abb. 42.

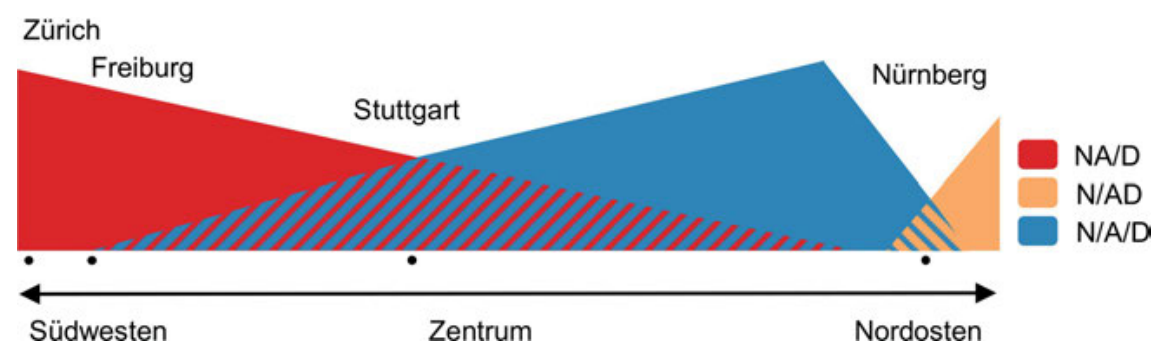

Abb. 44: Abstrahierte Darstellung der Übergangsstrukturen im Untersuchungsgebiet. ${ }^{96}$

Die Abbildung gibt die oben angeführten Erkenntnisse zur kasusmorphologischen Raumgliederung wieder: Der Verlauf der Achse - angedeutet durch die darauf liegenden Städte - zeigt die Südwest-Nordost-Gliederung, die für den oberdeutschen Raum angesetzt wurde. Diese Gliederung unterteilt drei größere Räume. Im Südwesten dominiert das synkretische Muster NA/D, im Osten das ebenfalls synkretische Muster N/AD. Dazwischen befindet sich ein Gebiet, in dem das Muster N/A/D zum östlichen Zentrum hin zunehmend prominenter wird. Während sich zum Südwesten und Westen hin der graduelle, geographisch recht breite Übergang einer schiefen Ebene abzeichnet, deutet sich nach Osten eine Übergangsstufe an.

Die Besonderheit der Übergangsstrukturen bei der kasusmorphologischen Raumgliederung liegt nun darin, dass sie auf verschiedenen Abstraktionsebenen zu erkennen sind: Sie lassen sich erstens, wie bereits beschrieben, auf Ebene der Kategorie Maskulinum erkennen. Entlang der in (13) angeführten Skala der Wortarten nimmt das Muster N/A/D vom Kerngebiet der vollen Distinktion nach Süden, Norden und Westen hin zu. Nach Osten hin tritt bei allen Wortarten mit Ausnahme des Personalpronomens die Stufe zwischen voller Distinktion und dem Muster N/AD zu Tage. Die geographische Übergangsstruktur ist hier demnach tatsächlich durch syntaktische Restriktionen konditioniert, die entlang der

96 Aus Gründen der Übersichtlichkeit wurde in dieser Abbildung auf die gesonderte Darstellung der Charakteristika aus Seiler (2005: 332) verzichtet. 
schiefen Ebene zu- beziehungsweise abnehmen. Während die volle Distinktion im äußersten Südwesten lediglich beim maskulinen Personalpronomen auftritt, findet sie sich im zentralen Gebiet auch beim Demonstrativpronomen und im Kerngebiet der vollen Distinktion bei allen untersuchten maskulinen Kasusmarkern mit Ausnahme des Adjektivs.

Die Übergangsstrukturen zeigen sich aber zweitens auch auf Ebene einzelner maskuliner Kasusmarker, wie beispielsweise dem maskulinen Adjektiv, beim Definit- oder Indefinitartikel (und in den Daten der Ortsgrammatiken auch beim Possessivum). Sie sind also nicht nur im Vergleich der Karten in 4.3.2, sondern auch innerhalb der Einzelkarte selbst zu erkennen. Auch hier zeigt sich beispielsweise eine allmähliche Abnahme von Belegen des Musters NA/D auf der Achse vom Südwesten hin zum Kerngebiet der vollen Distinktion. Während im Südwesten ausschließlich Belege für NA/D und in diesem Kerngebiet ausschließlich solche für N/A/D auftreten, zeigen die Karten zu beiden Datengrundlagen dazwischen einen Raum, in dem für einzelne Ortspunkte beide Muster für eine Wortart belegt sind, wo also auch auf Ebene des einzelnen maskulinen Kasusmarkers intrasystematische beziehungsweise idiolektale Variation auftritt (vgl. dazu das Beispiel in Tab. 26).

Die Übergangsstrukturen sind folglich auch auf dieser Abstraktionsebene grundlegend $\mathrm{zu}$ erkennen. Inwiefern diese Struktur der intrasystematischen bzw. idiolektalen Variation auf Ebene einzelner Wortarten jedoch alle Charakteristika einer schiefen Ebene aufweist, insbesondere inwiefern es auch hier tatsächlich (syntaktische oder andere) Restriktionen gibt, die das Auftreten der einzelnen Muster der Kasusmarkierung steuern, gilt es im Folgenden (vgl. 4.3.4) noch zu untersuchen.

Auf Basis der bisherigen Forschungsliteratur zu dieser Form der Mustervariation auf Wortartenebene, die sich jedoch nie explizit mit dem Untersuchungsraum, sondern eher mit kleinräumigen Systemen wie im Südhessischen, im Hochund Höchstalemannischen beschäftigt hat, lassen sich hierfür weitere Restriktionstypen zumindest vermuten. Neben den in Seiler (2005: 331) angeführten syntaktischen Restriktionen können hier durchaus auch semantische Faktoren, wie beispielsweise Agentivität oder Belebtheit oder auch die morphologische Struktur der Kasusmarker Einfluss auf das Auftreten eines bestimmten Musters in einem variierenden Teilsystem üben.

Zusammenfassend lässt sich die die kasusmorphologische Gliederung des oberdeutschen Raums wie folgt charakterisieren: Sie zeigt sich nicht nur bei einzelnen das Kasussystem betreffenden Phänomenen, sondern auch, wie bereits in Shrier (1965) beschrieben, in der Systemfunktion, d. h. bei vollständigen Mustern der Kasusmarkierung. Dabei lässt sich weder eine klare Ost-West- noch eine NordSüd-Gliederung beschreiben. Vielmehr zeigt sich ganz grundlegend eine Südwest- 
Nordost-Gliederung der verschiedenen Teilsysteme, die insbesondere im zentralen Bereich von einem recht komplex gestaffelten Distinktionsgebiet geprägt ist. Die Übergangsräume zwischen den so gegliederten Teilsystemen im oberdeutschen Raum lassen sich nicht durch das traditionelle Konzept der Isoglossen charakterisieren, sondern zeigen verschiedene, aus der Dialektsyntax bekannte Übergangsstrukturen, ${ }^{97}$ die sich jedoch je nach prominentem Muster im jeweiligen Teilsystem unterscheiden: der Übergang zwischen dem zentralen Gebiet der vollen Distinktion zu dem Teilsystem, in dem N/AD dominiert, zeigt eine Stufenstruktur, der zu dem Teilsystem, in dem NA/D dominiert, hingegen eine schiefe Ebene. Beide Übergangsstrukturen spiegeln sich auf verschiedenen Abstraktionsebenen wider, sowohl auf Ebene der Kategorie Maskulinum (wo sie entlang der einzelnen Wortarten verläuft) als auch auf Ebene der einzelnen maskulinen Wortarten (wo sie in intrasystematischer bzw. idiolektaler Mustervariation innerhalb einzelner Wortarten mündet).

\subsubsection{Struktur des Übergangs: intrasystematische und idiolektale Variation}

Variation zwischen verschiedenen Mustern der Kasusmarkierung innerhalb eines Teilsystems - sowohl zwischen verschiedenen maskulinen Wortarten als auch auf Ebene der einzelnen maskulinen Wortart - wurde in 4.3.3 als charakteristische geographische Übergangsstruktur der kasusmorphologischen Raumgliederung beschrieben. Dieses Kapitel widmet sich eben dieser Mustervariation im Speziellen dem Typus der Variation, der auf Ebene der einzelnen maskulinen Wortart auftritt und dessen mögliche Restriktionen noch zu klären sind (vgl. für ein Beispiel Tab. 26).

Unter Berücksichtigung des grundlegenden Konzepts der schiefen Ebene in Seiler (2005) ist davon auszugehen, dass die einzelnen Muster der Kasusmarkierung im variierenden Bereich bestimmte zu- beziehungswiese abnehmende Restriktionen aufweisen: Neben Belegdichte und -häufigkeit (sowie Präferenz) wurden dort geographisch zunehmende syntaktische Restriktionen für bestimmte Muster als typisches Merkmal von Übergangsstrukturen in Untersuchungen zu syntaktischen Phänomenen aufgeführt. Angelehnt an die Terminologie in

97 Die hier beschriebenen Übergangsstrukturen sind zwar aus der Dialektsyntax bekannt, inwiefern sie jedoch ausschließlich auf morphosyntaktische Phänomene beschränkt sind, ist unklar. Fleischer (2019: 655) verweist auf die speziellen methodischen Eigenschaften morphosyntaktischer Projekte, die insbesondere die Analyse von intrasystematischer Variation begünstigen und damit, anders als traditionellere Projekte, grundsätzlich auf andere Raumstrukturen als Isoglossen ausgelegt sind. 
Seiler (2008: 382) würde man in diesem Fall von einer konditionierten Variation sprechen, bei der zwei Varianten innerhalb desselben Systems koexistieren, jedoch auf unterschiedliche Funktionen oder Kontexte verteilt sind. Diese konditionierte Variation unterscheidet sich von der deutlich selteneren freien Variation, in der die Varianten zufällig verteilt sind, eben durch diese gesteuerte Distribution (vgl. Seiler 2008: 381-383). Beim Typus der Mustervariation zwischen verschiedenen Wortarten können eben diese Wortarten als restringierende Faktoren klassifiziert werden. Ein Ort im Untersuchungsraum weist also zwei verschiedene Muster der Kasusmarkierung im Maskulinum auf, wobei diese, je nach geographischer Lage an verschiedene maskuline Wortarten geknüpft sind. Hierbei handelt es sich folglich um konditionierte Variation.

Inwiefern auch der Typus der Mustervariation auf Wortartenebene, d. h. im Paradigma einzelner Wortarten, konditioniert ist, soll im Verlauf des folgenden Teilkapitels geklärt werden. Die Analyse dieser Variation und ihrer möglichen Restriktionen erlaubt einen Einblick in gleich zwei grundlegende Aspekte des oberdeutschen Kasusdiasystems: Aus dialektgeographischer Perspektive ermöglicht sie eine fundierte Charakterisierung der Eigenschaften und Restriktionen dieser speziellen kasusmorphologischen Übergangsstruktur. Aus Perspektive der strukturellen Gliederung bietet sie ein optimales Versuchslabor für den Einfluss von nicht-morphologischen Faktoren auf die Kategorie Kasus:

Auf Ebene des einzelnen Kasusmarkers lässt sich hier der Einfluss von nichtmorphologischen Kategorien auf das untersuchte Kasusdiasystem ablesen. Die Muster der Kasusmarkierung in den nicht-variierenden Teilsystemen des gesamten Diasystems sind größtenteils von morphologischen Faktoren wie Genus, Numerus und Person (vgl. 4.2.3) oder speziell im Maskulinum von der Wortart (vgl. 4.2.4) und zu einem gewissen Ausmaß von der geographischen Lage (vgl. 4.3.1) abhängig. Semantische Einflüsse konnten in 4.2.3 nur indirekt, über die grammatischen Kategorien aufgezeigt werden. Lässt ein Teilsystem jedoch bei einer einzigen Wortart innerhalb einer Kategorie mehr als ein Muster der Kasusmarkierung zu, besteht die Möglichkeit, dass nicht-morphologische Faktoren die jeweilige Variante steuern und demnach auch direkt auf das Kasussystem wirken. Eine vertiefende Analyse der möglichen Konditionierungen der unterschiedlichen Muster in den variierenden Systemen verschafft damit Einblicke in die Sensitivität der Kategorie Kasus gegenüber eben diesen nicht-morphologischen Faktoren. Durch die Analyse der variierenden Muster können nun also vertiefende Erkenntnisse über einen möglichen direkten Einfluss von Syntax und der Semantik auf die Kasusdistinktion gewonnen werden.

In dieser Analyse konzentriere ich mich dabei auf die Mustervariation beim maskulinen Definitartikel. Wie bereits oben erwähnt, ist dieser Kasusmarker im Korpus äußerst frequent und dort sowie in den Ortsgrammatiken zugleich geo- 
graphisch recht homogen belegt (vgl. 4.3.2). Grundlegend zeigen sich im oberdeutschen Gebiet zwei verschiedene Variationsstrukturen, die jeweils an eines der beiden hier auftretenden Muster der Kasusmarkierung (NA/D oder N/AD) gekoppelt sind. ${ }^{98}$

In Abb. 35, die die Belege zum maskulinen Definitartikel aus den Ortsgrammatiken darstellt, ist $\mathrm{zu}$ erkennen, dass 9 Orte Mustervariation beim maskulinen Definitartikel zeigen. Ein Ort zeigt Variation zwischen der vollen Distinktion und dem synkretischen Muster N/AD und acht Orte variieren zwischen N/A/D und NA/D. Aus den Ortsgrammatiken selbst lassen sich jedoch kaum weitere Informationen $\mathrm{zu}$ dieser Mustervariation ermitteln (an dieser Stelle sei noch einmal auf die Problematisierung der Ortsgrammatiken als Datengrundlage in 3.2.2 verwiesen.). Lediglich Fischer (1999: 184-185) und Weber (1987: 172) verweisen für Zürich und Luzern (sowie die jeweilige Umgebung) auf phonologische Einflüsse, die bestimmte Formen und damit Muster der Kasusmarkierung konditionieren - ein Phänomen, das teilweise mit Formenvariation in hochalemannischen Dialekten in Verbindung gebracht wird (vgl. Meyer 1967), ${ }^{99}$ das jedoch unter Berücksichtigung der großen Formenvielfalt beim maskulinen Definitartikel im Untersuchungsraum der Korpusanalyse nicht als Restriktion infrage kommt. ${ }^{100}$

Auch im Korpus finden sich 13 Transkripte, für die nach der in 3.5.4 beschriebenen Methode Mustervariation nachgewiesen werden konnte. Hier zeigt sich an drei Orten die Variation zwischen der vollen Distinktion und N/AD und an zehn Orten Variation zwischen N/A/D und NA/D, wobei die volle Distinktion in diesen variierenden Systemen quantitativ jeweils dominiert. Gemittelt zeigt sie in diesen variierenden Teilsystemen einen Anteil von 63\% (vgl. Tab. 27). Lediglich im Teilsystem von Mönchsdeggingen/Nördlingen_1 liegt der Anteil der vollen Distinktion bei 48\%. In den übrigen Teilsystemen im Korpus lässt sich angelehnt an die Klassifikation in Ellsäßer (2020: 66) von kanonischer Distinktion ausgehen, bei der ein synkretisches Muster lediglich als Variante mit relativ niedrigem Anteil an der Gesamtbelegmenge auftritt. Da die Daten der Korpusanalyse allesamt geo-

98 Eine feinere, auch quantitativ basierte Typologie der im oberdeutschen Gebiet auftretenden Mustervariation findet sich in Ellsäßer (2020: 64-67).

99 Wobei die Beschreibungen sich jeweils stärker auf Formen- als auf Mustervariation konzentrieren.

100 Dieses Phänomen erklärt lediglich das phonologisch determinierte Auftreten einer derForm im Akkusativ, das in den Fällen, in denen der auch die Nominativform darstellt, zu Synkretismus führt. In den variierenden Systemen tritt jedoch durchaus Synkretismus auf, der nicht auf diesen Formen beruht (z. B. de, de/ em, Frauenriedhausen/Dillingen), was eine solche phonologische Konditionierung in diesen Systemen eher unrealistisch macht. 
graphisch nah am Kerngebiet der vollen Distinktion liegen, stützt die anteilige Dominanz des distinkten Musters die Konzeptualisierung der schiefen Ebene in Seiler (2005), wonach die Häufigkeit eines Musters mit zunehmender Entfernung vom entsprechenden Pol abnimmt.

Tab. 27: Anteile verschiedener Muster in Transkripten mit variierenden Teilsystemen am Beispiel des maskulinen Definitartikels.

\begin{tabular}{lrrr}
\hline Sigle & Belege N/A/D & Belege N/AD & Belege NA/D \\
\hline Altheim/Ulm & $45,5^{101}$ & 0 & 32,5 \\
\hline Ballersdorf/Neuburg & 18,5 & 9,5 & 0 \\
\hline Bondorf/Böblingen & 28 & 0 & 9 \\
\hline Emerkingen/Ehingen & 26,5 & 0 & 13,5 \\
\hline Frauenriedhausen/Dillingen & 41,5 & 0 & 20,5 \\
\hline Hayingen/Münsingen & 28 & 0 & 26 \\
\hline Heimsheim/Leonberg & 12,5 & 0 & 9,5 \\
\hline Herkheim/Nördlingen & 25,5 & 8,5 & 0 \\
\hline Isingen/Balingen & 44,5 & 0 & 18,5 \\
\hline Meßbach/Künzelsau & 18 & 0 & 11 \\
\hline Mönchsdeggingen/Nördlingen_1 & 31,5 & 0 & 34,5 \\
\hline Waldmannshofen/Mergentheim_2 & 16,5 & 10,5 & 0 \\
\hline Wengen/Kempten & 34,5 & 0 & 19,5 \\
\hline
\end{tabular}

Im Korpus lassen sich die unterschiedlichen Muster jedoch nicht nur quantifizieren. Die Daten bieten zudem ausreichend viel Kontext und sind bereits ausreichend nach verschiedenen Variablen annotiert (3.5.1), um auch verschiedene, möglicherweise konditionierende Faktoren der Mustervariation direkt zu überprüfen. Während, wie bereits in 3.3.2 argumentiert, die Mustervariation in den Daten der Ortsgrammatiken lediglich auf nicht näher definierte intrasystematische Variation schließen lässt, zeigt das Auftreten dieser Struktur im Korpus, dass es sich hierbei um idiolektale Variation handelt. Es lässt sich daher ausschließen, dass variierende Muster durch eine unterschiedliche Ausprägung der

101 Zur Berechnung der Belegzahlen der einzelnen Muster s. 3.5.4. 
Kasusdifferenzierung verschiedener SprecherInnen am jeweiligen Ort verursacht ist. Es scheint vielmehr ein strukturelles Phänomen innerhalb des idiolektalen Systems einzelner SprecherInnen darzustellen.

Die folgende Tabelle gibt einen quantitativen Überblick über die Belegmenge in den verschiedenen variierenden idiolektalen Systemen im Korpus sowie über Belegmenge der einzelnen Muster (auf Grundlage des Vorgehens in 3.5.4) gegliedert nach den jeweiligen Transkripten. In Anhang 6.2.4 findet sich eine weitere Tabelle, die zusätzliche Informationen zu den variierenden Teilsystemen (wie beispielsweise Sprechlage und Alter der Gewährsperson und TranskriptorIn des Transkripts) listet. ${ }^{102}$

Neben seiner bereits oben erwähnten hohen Frequenz bietet sich der maskuline Definitartikel auch deshalb für eine vertiefende Analyse an, weil sich die meisten Arbeiten, die sich mit der Konditionierung von intrasystematischer Variation im Kasussystem auseinandersetzen, mit eben dieser Wortart beschäftigen (vgl. beispielsweise Meyer 1967, Weber 1987, Dal Negro 2004, Dirani 2020), ${ }^{103}$ sodass hier zumindest für bestimmte Variationsstrukturen bereits Ausgangshypothesen in der Literatur vorhanden sind, die im Folgenden anhand der Korpusdaten als mögliche Restriktionen für die (variierenden) Muster der Kasusmarkierung im Untersuchungsgebiet überprüft werden.

Eine Hypothese, die sich auf Basis dieser Arbeiten aufstellen lässt, ist daher, dass es sich bei der Übergangsstruktur hin zum in der vorliegenden Arbeit beschriebenen zentralen Distinktionsgebiet auch auf Ebene des einzelnen Kasusmarkers nicht um freie, sondern um konditionierte Variation handelt, dass die Muster demnach nicht zufällig, sondern gebunden an bestimmte funktionale oder formale Restriktionen verteilt sind.

Zwar bezieht sich keine dieser Arbeiten auf den Untersuchungsraum der Korpusanalyse, doch bieten sie allesamt Vorschläge für sprachsysteminterne konditionierende Faktoren intrasystematischer Variation, die in anderen oberdeutschen Gebieten auftreten, und zeigen damit durchaus realistische Szenarien auch für den hier untersuchten Raum auf. Dabei konzentrieren sie sich jedoch ausschließlich auf Systeme, die das synkretische Muster NA/D zeigen, weshalb die westliche Variationsstruktur, die dieses Muster einschließt, auch hier im Fokus steht.

102 Diese Information ist deshalb relevant, weil sie zeigt, dass die Transkripte mit variierenden Systemen von unterschiedlichen TranskriptorInnen erstellt worden sind. Die Variation lässt sich also nicht auf TranskriptorInneneffekte zurückführen.

103 Nur äußerst wenige Arbeiten, wie beispielsweise die von Rohdenburg (1993) zum niederdeutschen Pronomen, beziehen sich bei diesem Phänomen auf andere Wortarten. 
Bereits in 2.2.2.5 wurde eine gewisse Interaktion semantischer Faktoren mit der Kategorie Kasus beschrieben. Diese zeigt sich beispielsweise im Zusammenhang der Kategorie Genus (die ja großen Einfluss auf die Kasusdistinktion ausübt) mit bestimmten Belebtheitsregularitäten, der in Alber/Rabanus (2011) beschrieben wird. Zudem wirkt sich Belebtheit auch über die Personendistinktion auf die Kasusmarkierung aus: Während die 1. Person ausschließlich SELF ausdrückt und so immer die höchste Stufe der Belebtheitshierarchie einnimmt, kann die 3. Person sowohl die Stufen Human und Animate, als auch InANimate, ABSTRACT, MASS und LOCATION ausdrücken. Die Ausprägungen der Kategorie Person korrelieren folglich mit unterschiedlichen Belebtheitsstufen (vgl. 4.2.3).

Insbesondere für alemannische Dialekte wurde daneben auch eine direkte semantische Konditionierung für Variationsphänomene in den Mustern der Kasusmarkierung vorgeschlagen. Die Arbeit von Dal Negro (2004) zum maskulinen Definitartikel im Südwalser Ort Issime wurde in 2.2.2.5 bereits erwähnt: Dort beschreibt sie ein System, in dem Nominativ und Akkusativ ursprünglich durch distinkte Formen ([NoM] dar/ [AKK] da, dan) ausgedrückt wurden - eine kasusmorphologische Distinktion, die jedoch bereits vollständig nivelliert wurde ([Nom] dar, [AKK] dar). Diachron ließe sich daraus folglich eine Entwicklung vom Muster N/A/D hin zu NA/D ableiten. Die Form, die in diesem System ursprünglich einen distinkten Akkusativ ausgedrückt hat, ist mit diesem formalen Synkretismus jedoch nicht vollständig aus dem System verschwunden. Dal Negro (2004: 108-109) beschreibt spezielle Kontexte, definiert demnach semantische (und referentielle) Restriktionen, die das Auftreten der getilgten distinkten Akkusativform in diesem System reglementieren: Diese Formen treten deutlich häufiger bei unbelebten Referenten auf, während die ursprüngliche Nominativform eher bei belebten Referenten auftritt, was Dal Negro (2004: 108) durch die Verknüpfung von Belebtheit und Agentivität und damit verbundener prototypischer Subjektsinterpretation erklärt. Sie geht demnach von einem direkten Einfluss der Belebtheit des jeweiligen Referenten auf die Form des Kasusmarkers und damit implizit auf das Muster der Kasusmarkierung aus.

Für die alemannischen Dialekte wird an dieser Stelle häufig eine Form der differentiellen Objektmarkierung angenommen. Dieses Konzept besagt, dass weniger prototypische Objekte in transitiven Sätzen - d.h. solche, die belebt und agentivisch sind - eher eine distinkte Kasusmarkierung verlangen als prototypischere - d.h. solche, die unbelebt und eher patientivisch sind (vgl. dazu 2.2.2.5). Besteht in einem System demnach die Möglichkeit der Variation zwischen verschiedenen Mustern der Kasusmarkierung innerhalb einer grammatischen Kategorie oder eben einer Wortart, müsste man nach diesem Prinzip davon ausgehen, dass eine distinkte Akkusativmarkierung in den Kontexten auftritt, in denen das direkte Objekt belebt und/oder agentivisch und damit 
nicht prototypisch ist. Im Beispiel des von Dal Negro (2004) beschriebenen Südwalser Systems würde man folglich das Muster NA/D bei einem unbelebten Objekt und das Muster N/A/D bei einem belebten Objekt erwarten. Auf diese Weise würde Kasusdistinktion die Argumentstruktur eindeutig markieren. In den Kontexten, in denen diese Markierung jedoch durch andere, z. B. semantische Mittel, wie prototypischen Belebtheitsrelationen gewährleistet ist, wären in einem solchen System durchaus synkretische Muster möglich.

Neben Dal Negro (2004) setzt auch Baechler (2018) unter anderem differentielle Objektmarkierung für bestimmte Teile höchstalemannischer Systeme an. Neben einer durchgängigen Tendenz zum Nominativ-Akkusativ-Synkretismus sowie einer grundlegenden paradigmatischen Differenzierung menschlicher und nicht-menschlicher Referenten beschreibt sie dort die starke Präferenz der distinkten Akkusativ- und damit Objektmarkierung bei menschlichen Referenten (Baechler 2018: 325-328). Außer für die höchstalemannischen Dialekte wird ein solcher direkter Einfluss der Belebtheit auf die Muster der Kasusmarkierung lediglich im Nordniederdeutschen beschrieben (vgl. Rohdenburg 1998), wobei das dort beschriebene Paradigma weniger Kasusdistinktionen im engen Sinne als vielmehr ausschließlich Belebtheitsdifferenzen an sich zu kodieren scheint. Inwiefern es sich bei einer belebtheitsinduzierten Mustervariation auch um ein realistisches Szenario für die variierenden Teilsysteme im hier untersuchten Korpus handelt, kann im Rahmen der Analyse anhand verschiedener Variablen quantitativ überprüft werden:

Ein messbarer direkter Einfluss der Belebtheit auf die Kasusdistinktion zeigt sich nicht. In den 10 Transkripten, die im Korpus Variation zwischen den Mustern N/A/D und NA/D zeigen, wo das synkretische Muster also die formale Distinktion von Subjekt und direktem Objekt beeinflusst und damit Belebtheitsdistinktionen für diese Zuordnung relevant sein können, zeigt sich kein signifikanter Unterschied im Anteil distinkter und synkretischer Akkusativmarkierungen bei menschlichen oder allgemein belebten (15 distinkte und 7 synkretische Akkusativbelege) im Vergleich zu nicht belebten Referenten (51 distinkte und 27 synkretische Akkusativbelege). ${ }^{104}$ Ähnlich verhält es sich hier im Nominativ. Auch hier gibt es keine signifikante Differenz im Anteil von Synkretismus und Distinktion bei menschlichen oder allgemein belebten (100 distinkte und 34 synkretische Nominativbelege) und nicht belebten Referenten (33 distinkte und 15 synkretische Nominativbelege). ${ }^{105}$

104 Ein entsprechender $\chi^{2}$-Test zeigt einen $p$-Wert von $1(\mathrm{Chi}=3,0326 \mathrm{e}-30, \mathrm{df}=1)$ und mit Cramer'sV $=0,024$ eine geringe Effektstärke.

105 Ein entsprechender $\chi^{2}$-Test zeigt einen $p$-Wert von $0,55(\mathrm{Chi}=0,5498, \mathrm{df}=1)$ und mit Cramer'sV $=0,081$ eine geringe Effektstärke. 
Es zeigt sich keine signifikante Assoziation zwischen distinkten Akkusativformen und belebten Referenten beim direkten Objekt (12 distinkte und 7 synkretische Belege) im Gegensatz zum unbelebten direkten Objekt (28 distinkte Belege und 17 synkretische Belege). ${ }^{106}$ Auch konnte kein Zusammenhang zwischen agentivischen - also eher nicht prototypischen - und distinkten Akkusativen festgestellt werden, da es in den variierenden Teilsystemen im Korpus schlichtweg keine Belege für ein Proto-Agens im Akkusativ gibt. Akkusativformen drücken hier lediglich das ProtoPatiens, in einem Fall einen Proto-Rezipienten sowie Proto-Location aus, wobei sich dort (wie auch im Nominativ, wo jedoch durchaus Agenzien ausgedrückt werden) kein signifikanter Zusammenhang mit Kasussynkretismus oder -distinktion zeigt. $^{107}$

Generell deutet sich in den Korpusdaten an, dass die Markierung der Argumentstruktur - sowohl auf semantischer als auch auf syntaktischer Ebene - zumindest nicht messbar als Faktor für die Konditionierung der Mustervariation herangezogen werden kann. In Ellsäßer (2020: 74-75) habe ich auf Basis eines kleineren, vorläufigen Korpus die Vermutung angetellt, dass diejenigen Akkusativformen, die in funktional eher schwach belasteten Kontexten, d. h. außerhalb der Argumentstruktur auftreten, eher zu Synkretismus neigen. Dies konnte auf Basis der vollständigen und überarbeiteten Korpusdaten nicht bestätigt werden (direktes Objekt: 40 distinkte und 24 synkretische Belege, kein direktes Objekt: 24 distinkte und 10 synkretische Belege). ${ }^{108}$

Auch scheint Transitivität keinen Einfluss auszuüben. So wäre es beispielsweise denkbar gewesen, dass Nominativformen, die in intransitiven Relationen auftreten, einem Ökonomieprinzip folgend eher zu Synkretismus neigen könnten als solche, die in transitiven Relationen auftreten, wo folglich die Notwendigkeit besteht, Subjekt und Objekt distinkt $\mathrm{zu}$ markieren. Ein signifikanter Zusammenhang zwischen distinkter Nominativmarkierung und transitiven Relationen konnte allerdings ebenfalls nicht nachgewiesen werden (34 distinkte und 11 synkretische Nominativbelege in transitiven und 98 distinkte und 37 synkretische Belege in intransitiven Relationen). ${ }^{109}$

106 Ein entsprechender $\chi^{2}$-Test zeigt einen $p$-Wert von $0,56(\mathrm{Chi}=0,33382, \mathrm{df}=1)$ und mit Cramer'sV $=0,058$ eine geringe Effektstärke.

107 Ein entsprechender $\chi^{2}$-Test zeigt einen $p$-Wert von $1(\mathrm{Chi}=0,33382, \mathrm{df}=1)$ und mit Cramer'sV $=0,013$ eine geringe Effektstärke.

108 Ein entsprechender $\chi^{2}$-Test zeigt einen $p$-Wert von $0,56(\mathrm{Chi}=0,33382, \mathrm{df}=1)$ und mit Cramer'sV $=0,081$ eine geringe Effektstärke.

109 Ein entsprechender $\chi^{2}$-Test zeigt einen $p$-Wert von $0,85(\mathrm{Chi}=0,037879, \mathrm{df}=1)$ und mit Cramer'sV $=0,029$ eine geringe Effektstärke. 
Auch ein Zusammenhang zwischen Kasusdistinktion und weniger prototypischen Wortfolgevarianten, der aufgrund der funktionalen Interaktion des Kasussystems mit der Syntax (vgl. 2.2.2.4) durchaus nachvollziehbar gewesen wäre, konnte bei den variierenden Systemen des maskulinen Definitartikels im Korpus nicht nachgewiesen werden. So treten beispielsweise bei der Wortfolge $\mathrm{O}>\mathrm{S}$ distinkte und synkretische Akkusativformen (3 distinkte und 4 synkretische Belege bei $\mathrm{O}>\mathrm{S}$ ) sowie distinkte und synkretische Nominativformen auf ( 9 distinkte und 2 synkretische Belege bei $\mathrm{O}>\mathrm{S}$ ). ${ }^{110}$

Auch wenn semantische Regularitäten sowie bestimmte Eigenheiten der Argumentstruktur indirekt demnach einen gewissen Einfluss auf das Kasusdiasystem ausüben (vgl. 4.2.3), scheint sich dieser auf Ebene einzelner Kasusmarker nicht direkt in den Mustern der Kasusmarkierung abzuzeichnen. Die Korpusdaten zeigen keine Anhaltspunkte für eine funktionale Konditionierung der Mustervariation im Untersuchungsgebiet.

Dies lässt sich auch in den östlichen Systemen vermuten, die die Mustervariation zwischen N/A/D und N/AD aufweisen, zu deren möglicher Konditionierung die Forschungsliteratur bislang keine Hypothesen bietet. Hier wäre auf der Ebene der Argumentstruktur beim syntaktischen Muster nicht die kasusmorphologische Unterscheidung zwischen Subjekt und Objekt, sondern zwischen direktem und indirektem Objekt betroffen. In keinem der Teilsysteme, die diese Mustervariation im Korpus zeigen, ist ein indirektes Objekt belegt. Der Dativ tritt in den entsprechenden Transkripten lediglich als peripherer Kasus auf. Daher lässt sich die differenzierte Markierung der Argumentstruktur hier nicht direkt untersuchen. Da jedoch trotz der ausschließlich außerhalb der Argumentstruktur befindlichen Belege Mustervariation auftritt, kann diese auch nicht an das indirekte Objekt oder dessen semantische und syntaktische Eigenschaften gekoppelt sein. Dieses Ergebnis hat sich bereits durch primär syntaktisch orientierte Arbeiten angedeutet: Rauth (2016: 127-130) beobachtet für die Systeme, die das Muster N/AD zeigen, eine große Abfolgevarianz von direktem und indirektem Objekt, was nicht für eine Interaktion von Kasus und Wortfolge zur Disambiguierung der Argumentstruktur in diesem Fall spricht.

Neben einer syntaktischen oder semantischen Konditionierung der Mustervariation im Korpus ist aber auch eine formale Konditionierung der Muster denkbar, die an die jeweilige morphologische Struktur gekoppelt ist. In 4.2.2 wurde gezeigt, dass der Typus der Kasusmarkierung der kasustragenden Wort-

110 Ein statistischer Test erübrigt sich hier aufgrund der äußerst geringen Belegzahlen. Es zeigt sich jedoch, dass synkretische Belege grundsätzlich auch bei der Abfolge $\mathrm{O}>\mathrm{S}$ möglich sind, obwohl auch hier durchaus belebte Objekte auftreten. 
art durchaus Auswirkungen auf Kasusdistinktion und -synkretismus im Diasystem zu haben scheint. Suppletive Typen neigen eher zu Kasusdistinktion als affigierte. Im Rahmen einer recht einfachen Operationalisierung wurde dort gezeigt, dass klitische Formen des Definitartikels im Korpus signifikant häufiger mit distinkter Kasusmarkierung assoziiert sind als nicht-klitische Formen. Für den Definitartikel (wie für den Indefinitartikel) wurde ein relativ breites Spektrum von Typen angesetzt. Bei vollen und reduzierten Formen tritt hier die Affigierung eines Einzellautes ähnlich wie beim Demonstrativpronomen auf, bei (der Minimalhypothese entsprechenden) klitischen Formen kann partielle oder auch totale Suppletion auftreten. ${ }^{111}$ Angelehnt an den Zusammenhang zwischen suppletiven und eher klitischen Formen und Kasusdistinktion könnte nun angenommen werden, dass die jeweiligen Typen der Kasusmarkierung konditionierende Faktoren für die Muster der Kasusmarkierung speziell in den variierenden Teilsystemen darstellen.

Tatsächlich finden sich in der Forschungsliteratur, wie bereits angeführt, Anhaltspunkte dafür, dass in einigen Dialekten mindestens zwei Paradigmen bei den Definitartikeln angesetzt werden müssen (vgl. beispielsweise Studler 2013 zum Alemannischen, Dirani 2020 zum Südhessischen und Weiß/Dirani 2019 kontrastiv zum Südhessischen und Bairischen), die sich durch Formenreduktion unterscheiden. Inwiefern sich diese Differenzierung jedoch auf die Muster der Kasusmarkierung, die sich aus den einzelnen Paradigmen ablesen lassen, auswirkt, ist aus diesen Arbeiten nicht eindeutig ersichtlich. So zeigt sich beispielsweise in den alemannischen Paradigmen von Studler (2013: 152153) kein Unterschied in den Mustern bei vollen und reduzierten Formen, während die südhessischen Paradigmen bei Weiß/Dirani (2019: 314) bei Vollformen das Muster N/A/D und bei reduzierten Formen das Muster NA/D aufweisen und damit der oben ausgeführten, für das Untersuchungsgebiet der Korpusanalyse angesetzten Annahme eher widersprechen. Die Distinktion zwischen den Paradigmen der vollen und reduzierten Formen wird dabei häufig auf eine Ausdifferenzierung von semantischer und pragmatischer Definitheit zurückgeführt.

Aufbauend auf der Definitheitsskala von Himmelmann (1997: 23-27) setzt Nübling (2005: 108-112) verschiedene Domänen für klitische und volle Artikel an: Die Domäne des klitischen Artikels setzt sie im semantisch definiten Bereich, wo der Referent des Artikels ohne Kenntnis des Kontexts oder der Situation ausgemacht werden kann, an (z. B. Ich gehe in's Kino.). Den vollen Artikel, wie auch das Demonstrativpronomen, setzt sie im Bereich der pragmatischen Definitheit an,

111 Auf die Problematik bei der Untersuchung eines solchen Zusammenhangs auf Basis der Korpusdaten und des Untersuchungsdesigns wurde in 4.2.2 bereits verwiesen. 
wo der Referent kontext- beziehungsweise situationsgebunden ist (z. B. Ich gehe in das Kino im Kreuzviertel.) (vgl. Nübling 2005: 109 und Löbner 1985: 298). Weiß/Dirani (2019: 316) differenzieren diese Untergliederung feiner aus und beziehen sie explizit auf die südhessischen Artikelparadigmen, die, wie oben erwähnt, auch eine gewisse Mustervariation aufweisen. Eine Konditionierung der Muster im Untersuchungsgebiet der Korpusanalyse durch die verschiedenen Definitheitstypen und die damit verknüpfte Differenzierung von vollen und reduzierten Formen soll daher ebenfalls überprüft werden.

Da in der Literatur ein Zusammenhang zwischen semantischer Definitheit und Klise angeführt wurde, wurde im Korpus zudem ein Zusammenhang zwischen semantischer Definitheit und den entsprechenden Mustern der Kasusmarkierung getestet. ${ }^{112}$ In den westlichen Systemen, die zur Variation mit dem Muster NA/D neigen, hat sich jedoch weder bei den Akkusativbelegen (pragmatische Definitheit 49 distinkte und 24 synkretische und semantische Definitheit 19 distinkte und 10 synkretische Belege) noch bei den Nominativbelegen (pragmatische Definitheit 80 distinkte und 33 synkretische und semantische Definitheit 72 distinkte und 26 synkretische Belege) ein Zusammenhang gezeigt. ${ }^{113}$

Es lässt sich hier jedoch auch ein Zusammenhang zwischen reduzierten beziehungswiese als klitisch klassifizierten Formen (die unter den in 4.2.2 ausführlich diskutierten, nicht optimalen Bedingungen klassifiziert wurden) und der Mustervariation beim maskulinen Definitartikel testen. Die Korpusdaten zeigen trotz der recht groben Operationalisierung von reduzierten Formen (vgl. 4.2.2), dass als klitisch klassifizierte Formen auch beim maskulinen Definitartikel in den variierenden Systemen tatsächlich seltener zu Synkretismus neigen als als nicht-klitisch klassifizierte Formen. Diese Assoziation ist jedoch nicht signifikant. ${ }^{114}$ Da sich dieser Zusammenhang allerdings übergreifend über alle Belege des Definitartikels im Korpus manifestiert hat, ist die Differenzierung

112 Semantische und pragmatische Definitheit wurden den Korpusdaten dazu soweit möglich auf Basis der Übersicht und der Beispiele in Nübling (2005: 108-112) zugeordnet. Da es sich hierbei um ein komplexes Unterfangen handelt, wurde diese Klassifikation nicht im gesamten Korpus, sondern nur stichprobenhaft für die Definitartikel der variierenden Systeme durchgeführt.

113 Ein entsprechender $\chi^{2}$-Test zeigt einen $p$-Wert von 0,56 $(\mathrm{Chi}=0,34523, \mathrm{df}=1)$ und mit Cramer'sV = 0,043 eine geringe Effektstärke.

114 Grundlage für den $\chi^{2}$-Test ist eine Kreuztabelle ähnlich der Tabelle zu Klitika in 4.2.2. Gewertet wurden die Belege des maskulinen Definitartikels Nominativ und Akkusativ in den variierenden zehn Systemen mit Muster NA/D. Der Residualwert der Assoziation von Klitika und Kasussynkretismus beträgt -1,4. Der p-Wert des Tests 0,1884 (Chi=1,7301, df =1). Der Test weit mit Cramer’sV = 0,098 eine geringe Effektstärke auf. 
zwischen vollen und klitischen Formen als speziell konditionierender Faktor der Mustervariation in den variierenden Systemen äußerst unwahrscheinlich.

In den östlichen Systemen, die Variation mit dem Muster N/AD zeigen, lässt sich auch dieser Zusammenhang kaum empirisch überprüfen. Mit lediglich 92 Belegen in diesen variierenden Systemen (23 im Nominativ, 19 im Akkusativ und 50 im Dativ) stellt das Korpus hierfür auch keine ausreichend große Datengrundlage zur Verfügung. Im Dativ dominiert sowohl bei als klitisch klassifizierten als auch bei als nicht-klitisch klassifizierten Belegen klar die Distinktion (30 distinkte und 9 synkretische klitische Belege, 11 distinkte und keine synkretischen nicht-klitischen Belege). Auffällig ist hier lediglich, dass als klitisch klassifizierte Akkusativbelege, entgegen der in 4.2.2 gesetzten Annahme, durchweg synkretisch auftreten - eine Beobachtung, die jedoch durchaus der geringen Datengrundlage von lediglich 7 entsprechenden Akkusativbelegen geschuldet sein kann. Bei den als nicht-klitisch klassifizierten Belegen dominiert auch hier die Distinktion mit 9 Belegen. Synkretismus ist lediglich zweimal belegt.

Zur Struktur im Osten des Untersuchungsgebiet, die den Übergang zwischen den Gebieten der Muster N/A/D und N/AD prägt, konnten die Korpusdaten der variierenden Systeme nur äußerst wenige Erkenntnisse liefern, was jedoch auch an dem offensichtlichen Charakteristikum dieses Übergangstypus liegt: der geringen geographischen Ausdehnung und der damit verbundenen niedrigen Belegzahl. Auch wenn die Grenzen zwischen beiden Systemen im Norden das Untersuchungsgebiet der Korpusanalyse durchkreuzen, konnten nur wenige Belegorte ausgemacht werden, die das spezielle variierende Übergangsmuster zeigen. Auch auf Grundlage dieser wenigen Daten kann jedoch angenommen werden, dass es sich bei diesem Muster um eine Form der freien Variation handelt, die sich auf Ebene der idiolektalen Systeme einzelner SprecherInnen in unmittelbarer geographischer Nähe zum jeweils anderen Gebiet äußert. Bereits oben wurde mit Dal (1971a: 188) vermutet, dass es sich hierbei nicht um eine Grenze zwischen zwei grundlegend verschiedenen kasusmorphologischen Systemen, sondern lediglich um die Grenze zwischen zwei phonologischen Ausdrucksformen desselben kasusmorphologischen Systems handelt, das jedoch von einer an der Systemfunktion orientierten Analyse, wie der hier durchgeführten, nicht erfasst wird. Dies würde nicht nur das östliche, für die 3. Person eher atypische Muster der Kasusmarkierung N/AD, sondern auch den Unterschied zwischen der Struktur dieses Übergangs und dem im Westen zwischen N/A/D und NA/D erklären. Dal (1971a) führt diesen Gedankengang jedoch nicht weiter aus und bleibt damit ein mögliches Szenario über die zugrundeliegenden (morphophonologischen) Prozesse schuldig.

Der Fokus der Analyse lag in diesem Teilkapitel auf der geographisch deutlich weiter ausgedehnten westlichen Übergangsstruktur, die zur Mustervariation 
zwischen N/A/D und NA/D neigt. Die aufgrund der Erkenntnisse zu anderen ober- beziehungsweise hochdeutschen Systemen getesteten möglichen Restriktionen konnten für die variierenden Systeme im Untersuchungsgebiet der Korpusanalyse allesamt nicht bestätigt werden. Es besteht daher tatsächlich die Möglichkeit, dass es sich bei der im Übergangsgebiet auf Ebene der einzelnen Kasusmarker auftretenden Mustervariation zumindest aus Perspektive einer paradigmatischen Analyse um freie Variation handelt. Die westliche Variationsstruktur zeigt dabei auf dieser Ebene abgesehen von den zu- beziehungsweise abnehmenden Restriktionen alle Charakteristika der schiefen Ebene. Der Anteil des Musters N/A/D ist nahe am Distinktionsgebiet recht hoch. Die Belegdichte des Musters nimmt mit zunehmender Entfernung von diesem Gebiet ab.

Die Übergangsstruktur der schiefen Ebene ist folglich in der kasusmorphologischen Raumgliederung auf den unterschiedlichen Abstraktionsebenen, auf denen sie sich zeigt, unterschiedlich stark ausgeprägt: Auf beiden Ebenen der, des Maskulinums (mit allen seinen Wortarten) sowie der, einzelner maskuliner Wortarten - kristallisiert sich eine Abnahme der Belegdichte sowie anteilige Belegzahl des Musters der vollen Distinktion vom Kerngebiet hin nach Süden, Westen und Osten heraus. Während die Abnahme auf Ebene des Maskulinums mit einer zunehmenden Restriktion der Wortarten, in denen dieses Muster auftritt, einhergeht, zeigt sich auf Ebene eben dieser Wortarten scheinbar freie Mustervariation. Diese ist jedoch - ebenso wie die konditionierte Variation auf Ebene des Maskulinums - stark geographisch gebunden, bildet also einen Variationsraum zwischen dem Gebiet im Südwesten, in dem das synkretische Muster NA/D dominiert, und dem Kerngebiet der vollen Distinktion im westlichen Zentrum ab.

Die geographische Übergangsstruktur lässt sich anhand der Korpusdaten bis zum Einzeltranskript beziehungsweise bis zum einzelnen daraus rekonstruierten idiolektalen Kasussystem zurückverfolgen. Die Mustervariation in diesem Variationsraum manifestiert sich damit nicht zwischen einzelnen (diatopisch oder diachron unterschiedlich orientierten) SprecherInnen an einem Ort - in Form von intrasystematischer Variation - sondern im idiolektalen System einzelner Personen, aus dem sich für den einzelnen Kasusmarker unterschiedliche Paradigmen rekonstruieren lassen.

Inwiefern dieser Variationsraum ein stabiles System darstellt (vgl. Glaser 2017: 177), lässt sich auf Basis der hier genutzten Daten aus Ortsgrammatiken und dem Korpus von Ruoff (1984) nicht vollständig ermitteln. Ein mit Blick auf die diachrone Entwicklung des (ober)deutschen Kasussystems (vgl. 2.2.2.1) durchaus realtistisches Szenario, könnte man wie folgt skizzieren: Der Variationsraum spiegelt einen diachronen Übergang, bei dem die maskulinen Kategorien den Mustern der Kasusmarkierung der übrigen Kategorien der 3. Person (Femininum, Neutrum 
und Plural) NA/D, angeglichen werden. Diese diachrone Ausdehnung des Musters NA/D würde sich in diesem Fall geographisch von Südwesten nach Nordosten bzw. zum Kerngebiet der Distinktion entlang der einzelnen maskulinen Wortarten entwickeln, wobei sie sich innerhalb idiolektaler Systeme manifestiert, wo der Anteil des Musters NA/D sukzessive zu- und der Anteil des Musters N/A/D entsprechend abnimmt. Ein solches Szenario würde damit, so diese Entwicklung ungehindert voranschreiten würde, den vollständigen Rückzug der vollen Distinktion auf die Domäne der 1. (und womöglich 2.) Person Singular im oberdeutschen Diasystem vorhersagen.

Die Korpusdaten geben dabei zumindest einen kleinen Hinweis zur Antwort auf die Frage, inwiefern sich hier überhaupt eine diachrone Entwicklung zeigt und ob diese unter Berücksichtigung verschiedenster interagierender Faktoren voranschreitet: Aus sieben Orten liegen im Korpus je zwei Transkripte vor. Es wurden hier also die Muster zweier idiolektaler Systeme aus einem Ort rekonstruiert, die jeweils von einer älteren und einer jüngeren Person stammen. An diesen Orten kann eine sehr vereinfachte Apparent-time-Analyse konstruiert werden. An den zwei Ortspunkten, an denen sich die Muster der Kasusmarkierung zwischen diesen Transkripten unterscheiden, zeigt sich in den Transkripten, die den Idiolekt der älteren Personen abbilden (Hettlingen/Wertingen_1, geboren 1883 und Mönchsdeggingen/Nördlingen_2, geboren 1879), tatsächlich das als älter angesetzte Muster der vollen Distinktion N/A/D, während die Transkripte der jüngeren Personen das als progressiv eingeschätzte Muster NA/D (Hettlingen/Wertingen_2, geboren 1933) beziehungsweise die Mustervariation (Mönchsdeggingen/ Nördlingen_1, geboren 1937) zeigen. ${ }^{115}$ Es zeigt sich demnach tatsächlich ein erster Hinweis auf eine diachrone Ausdehnung des Musters NA/D in ursprüngliche N/A/D-Gebiete.

Auch unter Berücksichtigung der in 4.3.2 angesetzten Chronologisierung der Daten, die den Sprachstand, den die Ortsgrammatiken wiedergeben, als älter klassifiziert hat, könnte man eine diachron und geographisch fortschreitende Ausbreitung des Musters NA/D annehmen. In den Karten zu den Ortsgrammatiken weist das Muster N/A/D tatsächlich ein größeres geographisches Verbreitungsgebiet auf als in denen zu den Korpusdaten. Für vertiefende Erkenntnisse zur Diachronie der dialektalen Kasussysteme im oberdeutschen Raum wären jedoch Real- oder zumindest weitere Apparent-time-Analysen notwendig. ${ }^{116}$

115 Ein Vertikalisierungsphänomen kann hier jedoch nicht vermutet werden: Die Sprechlagen sind innerhalb dieser Belegorte konstant (vgl. Tab. 5).

116 Vertiefende Apparent-time-Analysen analog zu der hier an zwei Belegorten durchgeführten wären auf Basis der Daten der Tübinger Arbeitsstelle „Sprache in Südwestdeutschland durchaus möglich. Zwar sind in Ruoff (1984) nur für sieben Orte mehrere Transkripte enthal- 
Auf Basis der Daten dieser Arbeit kann die Frage nach der Ursache beziehungsweise der exakten Beschaffenheit des Variationsraums nicht vollständig geklärt werden. Es konnten aber erste, empirisch fundierte Hinweise für vertiefende Untersuchungen gegeben werden. So können etwa die in dieser Arbeit gewonnenen Erkenntnisse zur (variierenden wie konstanten) Kasusmarkierung von der paradigmatischen auch auf die syntagmatische Ebene übertragen und dort Mechanismen untersucht werden, die diese Variation unter Umständen ausgleichen oder aber noch verstärken.

\subsubsection{Zwischenfazit}

Dieses Kapitel hat die Erkenntnisse zur dialektgeographischen Gliederung der oberdeutschen Kasussysteme dargestellt, die auf Grundlage der beiden in der Analyse genutzten Datengrundlagen, der Ortsgrammatiken und des Korpus, gewonnen werden konnten. Es wurden ganz grundlegende Fragen nach Verbreitung und Struktur der kasusmorphologisch determinierten Gebiete gestellt und so einige weiße Flecken im Forschungsfeld der kasusmorphologischen Dialektgeographie erschlossen.

Dabei konnten die maskulinen Wortarten als raumbildende Faktoren im oberdeutschen Gebiet herausgearbeitet werden (4.3.1). Die geographische Gliederung, die durch diese Kategorien charakterisiert ist, lässt sich grundlegend durch bereits etablierte Modelle der morphosyntaktischen Raumgliederung beschreiben, wobei sich insbesondere die Übergangsstrukturen bei einer strukturell-dialektologischen Analyse vollständiger Muster der Kasusmarkierung auf mehreren Abstraktionsebenen spiegeln: Die charakteristischen Strukturen zeigen sich sowohl auf Ebene der einzelnen maskulinen Kasusmarker als auch übergreifend auf Ebene der Kategorie Maskulinum (4.3.2 und 4.3.3).

Ein besonderes Merkmal der kasusmorphologischen Raumbildung stellt die Mustervariation innerhalb der idiolektalen Systeme dar. Diese zeigt sich auf höherer Abstraktionsebene in der Variation zweier Muster der Kasusmarkierung, die durch die verschiedenen Wortarten konditioniert ist. Auf niedrigerer Abstraktionsebene äußert sie sich in der wahrscheinlich freien Variation der Muster bei einzelnen Kasusmarkern - ein Phänomen, das hier beispielhaft am maskulinen Definitartikel vorgestellt und näher untersucht wurde. Mustervariation manifestiert sich dabei nicht nur als kleinräumige Übergangsstruktur (wie beispielsweise

ten, grundsätzlich liegen jedoch für jeden Untersuchungsort mehrere Tonaufnahmen verschieden alter SprecherInnen vor. 
im Osten des Untersuchungsraums), sondern auch in einem äußerst großflächigen Variationsraum (im Zentrum und im Westen), der als eigenes kasusmorphologisches Teilsystem beschrieben werden kann (4.3.4).

In diesem Kapitel haben sich zudem die Vorteile einer Methodenkombination bei der dialektgeographischen Untersuchung von Kasussystemen gezeigt. Die Ergebnisse aus der Analyse der Ortsgrammatiken haben einen Überblick über nahezu den gesamten oberdeutschen Sprachraum ermöglicht, der vom kanonisierten Aufbau der Grammatiken und dem damit einhergehenden relativ homogenen Belegnetz, das auch eine geostatistische Analyse ermöglicht hat, profitiert. Die Nachteile dieser Datengrundlage zeigen sich jedoch erstens in den größtenteils auf einzelnen Nennformen beruhenden Beschreibungen, die weder Aufschluss über Frequenzverhältnisse noch über mögliche Interaktion des Kasusteilsystems mit nicht-morphologischen Faktoren bieten, und zum anderen im lockeren, nicht homogen verteilten Ortsnetz, das speziell im östlichen, aber auch im zentralen westlichen Oberdeutschen große Lücken aufweist.

Insbesondere Letzteres hat sich als kasusmorphologisch höchst spannendes Gebiet erwiesen und wurde vom recht feinmaschigen Ortsnetz des hier genutzten Korpus gut abgedeckt. Aufgrund der recht unterschiedlichen Ausschnitte aus dem idiolektalen System, das in den einzelnen Transkripten des Korpus abgedeckt wurde, war das Belegnetz des Korpus jedoch äußerst heterogen; bestimmte Wortarten waren in einigen Orten überdurchschnittlich frequent und in anderen wiederum nicht oder nur unzureichend belegt, weshalb trotz der hohen Belegzahl im Korpus insgesamt keine statistischen dialektgeographischen Methoden (z. B. Kartierung anhand einer Clusteranalyse) möglich waren. Die Korpusdaten haben jedoch frequenzbasierte Analysen ermöglicht und konnten so beispielsweise diejenigen Muster aufzeigen, die in den variierenden Teilsystemen dominieren. Zudem konnten die Kasusmarker hier nicht nur separat, sondern im Kontext analysiert werden, wodurch beispielsweise Analysen zu möglichen semantischen und syntaktischen Einflüssen angestellt werden konnten. Die dialektgeographischen Strukturen, die sich in den unterschiedlich definierten Teilsystemen in den Ortsgrammatiken gezeigt haben, konnten im Rahmen der Korpusanalyse zum einzelnen idiolektalen System zurückverfolgt werden. Trotz des heterogenen Belegnetzes hat sich die Korpusanalyse damit als lohnende Datengrundlage auch für dialektgeographische Fragestellungen erwiesen, deren Vorteile insbesondere bei der frequenzbasierten Analyse von Strukturen und Interaktionen verschiedener grammatischer Kategorien und Funktionen liegen. 


\subsection{Ausblick: Kasusmarkierung im Syntagma}

In dieser Arbeit habe ich ich mich bisher auf die paradigmatische synthetische Kasusmarkierung konzentriert. Den strukturellen Ausgangspunkt haben einzelne kasustragende Wortformen sowie die Muster der Kasusmarkierung im Paradigma gebildet, die auf Basis dieser Wortformen rekonstruiert werden konnten. Die Analyse hat sich damit auf die kleinste Einheit des Kasusdiasystems konzentriert und deren strukturelle sowie geographische Charakteristika im untersuchten Diasystem ausführlich dargestellt.

Es hat sich gezeigt, dass das Auftreten verschiedener Kasusmarker im untersuchten Diasystem auf die Kombination nominaler und konkordialer Kasusmarker im Deutschen zurückzuführen ist (vgl. 2.2.2.2). Die abstrakten Kasus einer Nominaloder Präpositionalphrase können so durch mehrere Wortformen (z. B. verschiedene Artikel, Adjektive oder Pronomen) ausgedrückt werden. In 4.2.4 wurden diese als zentrale und periphere Kasusmarker beschrieben, die zur Kasusdistinktion im Diasystem in unterschiedlichem Ausmaß beitragen, beziehungsweise aus diachroner Perspektive unterschiedlich stark von formalen Nivellierungstendenzen betroffen sind. Personalpronomen zeigen beispielsweise eher hohe Distinktionsquoten (sind folglich zumeist zentrale Kasusmarker), Adjektive hingegen eher niedrige (und sind durchweg periphere Kasusmarker). Diachron orientierte Arbeiten, wie beispielsweise Hotzenköcherle (1962b: 327) prognostizieren aufgrund dieser teilweise starken Nivellierungstendenzen bestimmter, im Diasystem peripherer Kasusmarker den Relevanzverlust oder sogar den vollständigen Abbau der Kategorie Kasus (vgl. 2.2.2.1). Prognosen wie diese nehmen jedoch eine ausschließlich paradigmatische Perspektive ein und konzentrieren sich zumeist nur auf bestimmte Ausschnitte des Kasusdiasystems, insbesondere auf Nomen.

Bislang nehmen noch relativ wenige Arbeiten auch eine syntagmatische Perspektive auf (kasus-)morphologische Phänomene ein (vgl. Schmidt et al. 2019: 35). Dabei weisen gerade die unterschiedlichen Nivellierungstendenzen der einzelnen Kasusmarker stark auf eine Interaktion dieser Marker auf syntagmatischer Ebene hin: Insbesondere die klammernden Verfahren in der deutschen Nominalphrase (vgl. dazu Ronneberger-Sibold 2010b) führen dazu, dass der abstrakte Kasus einer Nominal- oder Präpositionalphrase nicht mehr am Nomen selbst (vgl. 2.2.2.1), d. h. am rechten Klammerrand, sondern am häufig verstärkten (Ronneberger-Sibold 2010b: 103-105) linken Klammerrand und damit an Determinierern und Pronomen markiert wird. Ein einzelner abstrakter Kasus kann demnach grundsätzlich durch mehrere, verschiedene Marker innerhalb der Phrase ausgedrückt werden. Aus syntagmatischer Perspektive würde die vollständige Distinktion an allen kasustragenden Wortformen daher zu Redundanzen in der Kasusdistinktion führen. Die Nivellierung bestimmter Kasus- 
marker stellt aus dieser Perspektive einen ökonomischen Prozess dar (vgl. dazu Askedal 2001), der redundanter Kasusmarkierung vorbeugt.

Aufbauend auf der Grundlage, die die paradigmatische Analyse zahlreicher Kasusmarker in dieser Arbeit geschaffen hat, können nun größere strukturelle Einheiten, wie z. B. Phrasen oder ganze Sätze, in den Blick genommen werden. Hier ist es nun erstmals möglich, nicht nur bestimmte Ausschnitte, sondern das (nahezu) vollständige Kasusdiasystem, also unterschiedliche kasustragende Wortarten und deren syntagmatische Interaktion in gesprochensprachlichen Daten zu untersuchen. Dabei stellt sich die Frage, inwiefern die Kategorie Kasus in diesen größeren Einheiten den paradigmatischen Nivellierungstendenzen einzelner Kassusmarker zum Trotz distinkt markiert wird, ob die Nivellierungstendenzen folglich auf einen Verlust (vgl. Hotzenköcherle 1962b) oder auf einen Ökonomisierungsprozess (vgl. Askedal 2001) des Kasussystems hinweisen.

Dieses Kapitel hat nun zum Ziel, einen Ausblick auf eine solche empirische Untersuchung des Ausdrucks abstrakter Kasus im Syntagma zu geben. Es skizziert eine korpusbasierte Analyse von Kasusdistinktion auf Phrasen- und Satzebene, aufbauend auf Methodik, Daten und Erkenntnissen der (paradigmatischen) Korpusanalyse dieser Arbeit. In einer Stichprobe aus dem Transkript aus Alfdorf/Schwäbisch Gmünd, dem längsten Transkript in Ruoff (1984), das den größten Ausschnitt eines idiolektalen Systems im Korpus bietet, wird die Interaktion peripherer und zentraler Kasusmarker im Syntagma beispielhaft analysiert. Untersucht wurden die Phrasen aus dem Transkript, in denen ein Adjektiv belegt ist (118 Belege). Adjektive wurden in dieser Arbeit als periphere Kasusmarker mit einer niedrigen Distinktionsquote (durchschnittlich nur 8,9\%) klassifiziert. Es gilt nun zu ermitteln, inwiefern sich diese niedrige Distinktionsquote auf den distinkten Ausdruck des abstrakten Kasus in der entsprechenden Phrase im Allgemeinen und damit auch auf die distinkte Markierung der Argumentstruktur auf Satzebene auswirkt. Dabei stellt sich die Frage, ob durch die niedrige Distinktionsquote (strukturelle) kasusmorphologische Ambiguität entsteht, ob die synkretische Kasusmarkierung am Adjektiv durch andere Kasusmarker im Syntagma ausgeglichen wird, oder ob in diesen Fällen andere Kategorien - z. B. Kongruenz, Belebtheit oder Serialisierung - die Argumentstruktur disambiguieren und Synkretismus damit durch Mechanismen außerhalb der Kategorie Kasus ausgeglichen wird.

Für 99 der 118 Belege in der Analyse kann ein Muster der Kasusmarkierung des Adjektivs rekonstruiert werden; acht davon zeigen distinkte Kasusmarkierung, 91 sind synkretisch. Das Transkript von Alfdorf/Schwäbisch Gmünd bildet damit einen durchschnittlichen Wert (Distinktionsquote von $8,1 \%) \mathrm{ab}$. 
Die Analyse der synkretischen Adjektivbelege zeigt, dass sich der Kasussynkretismus häufig zwar an der einzelnen Wortform, aber nicht unbedingt innerhalb der gesamten kasustragenden Phrase äußert, dass der paradigmatische Synkretismus also innerhalb der Phrase ausgeglichen wird. Bei 29 Belegen (32\%) findet sich innerhalb derselben Phrase und damit für denselben abstrakten Kasus, eine weitere, distinkte Markierung, beispielsweise ein Artikel, ein anderes Adjektiv oder ein Possessivdeterminierer. Beispiel (14) illustriert diesen Typus der (syntagmatischen) Kasusmarkierung. Auf Grundlage der paradigmatischen Analyse wurde ermittelt, dass die Adjektivform alde im idiolektalen System von Alfdorf/Schwäbisch Gmünd synkretisch, der maskuline Definitartikel $d r$ hingegen distinkt ist. Es handelt sich in diesem Beispiel um zwei konkordiale Marker desselben abstrakten Kasus. Dieser wird trotz des synkretischen Adjektivs innerhalb der Nominalphrase und zwar am linken Klammerrand (vgl. Ronneberger-Sibold 2010b: 103-105) distinkt markiert.

$\begin{array}{llll}d r & \text { alde } & \text { Schlebber muaß älles schaffa. } \\ \text {,der_DIST } & \text { alte_syN } & \text { Schlepper muss alles arbeiten. }\end{array}$

Beleg 7912, Alfdorf/Schwäbisch Gmünd

Bei diesem Typus scheint das Diasystem ökonomischen Prinzipien zu folgen: Anstatt redundante Kasusmarkierung zu zeigen, was bei zwei konkordialen Markern durchaus möglich erscheint, wird der abstrakte Kasus der Phrase hier lediglich an einer Stelle, d. h. durch nur einen Marker distinkt markiert.

Auch in Belegen, in denen das synkretische Adjektiv nicht durch einen distinkten Kasusmarker innerhalb der Phrase ergänzt wird, wenn es sich um ein starkes Adjektiv handelt oder der ergänzende Kasusmarker ebenfalls synkretisch ist, lässt sich teilweise eine solche kasusmorphologische Ökonomie erkennen: Sie zeigt sich im distinkten Ausdruck der Argumentstruktur durch Kasusmarkierung, der trotz einer einzelnen synkretischen Phrase auf Satzebene gewährleistet ist. Bei 32 Belegen, bei denen die Phrase, die das Adjektiv enthält, synkretisch ist, wird der Kasus eines anderen Arguments im Satz distinkt markiert. Auch hier führt der einzelne synkretische Adjektivbeleg folglich nicht zu (struktureller) Ambiguität. Dieser Typus ist in (15) illustriert. Die synkretische Adjektivform andara wird hier durch den Indefinitartikel en ergänzt, der ebenfalls auf paradigmatischer Ebene als synkretisch klassifiziert worden ist. Der abstrakte Akkusativ, den die Nominalphrase en andara Schlebber trägt, wird demnach innerhalb der Phrase nicht distinkt markiert. Strukturelle Ambiguität wird in diesem Satz jedoch - neben der kongruierenden Verbform 
solldat - durch eine distinkte Nominativmarkierung der subjektivischen Nominalphrase verhindert. Das Personalpronomen mir stellt im Diasystem einen zentralen Kasusmarker mit äußerst hoher Distinktionsquote dar und markiert allein - nominal - den abstrakten Nominativ. Auf Satzebene ist das Subjekt hier daher durch eine distinkte Kasusmarkierung gekennzeichnet; die synkretische Markierung des Objekts gefährdet den distinkten Ausdruck der Argumentstruktur daher nicht. Auch in diesen Fällen werden die abstrakten Kasus im Satz damit distinkt markiert. \\ $\begin{array}{lllll}\text { mir } & \text { solldat halt en } & \text { andara } & \text { Schlebber ha. } \\ \text {,Wir_DIST } & \text { sollten halt } & \text { einen_SYN } & \text { anderen_SYN } & \text { Schlepper haben. }\end{array}$ \\ Beleg 7884, Alfdorf/Schwäbisch Gmünd}

Diese beiden Typen der distinkten syntagmatischen Kasusmarkierung auf Phrasen- oder Satzebene finden sich bei 67\% der Adjektivbelege, die im Transkript aus paradigmatischer Perspektive als synkretisch klassifiziert worden sind. Gemeinsam mit den Adjektiven, die auch auf paradigmatischer Ebene Kasusdistinktion aufweisen, sind damit 70\% der abstrakten Kasus, die im Transkript von Adjektiven getragen werden, durch Kasusmorphologie distinkt markiert. Die distinkte Markierung tritt dabei entweder direkt am Adjektiv, innerhalb der Nominal- beziehungsweise Präpositionalphrase oder innerhalb des Satzes auf. Von einem vollständigen Abbau oder gar einem Relevanzverlust der Kategorie Kasus im untersuchten Diasystem (vgl. Hotzenköcherle 1962b) kann auf Grundlage dieser Ergebnisse folglich keinesfalls ausgegangen werden. Zwar zeigt sich auf paradigmatischer Ebene durchaus eine starke Tendenz zur Nivellierung der Kasusdistinktion - was sich beispielsweise am großen Anteil der peripheren Marker (4.2.4) zeigt -, diese Nivellierung scheint jedoch einem ökonomischen Prinzip zu folgen, das Redundanzen auf syntagmatischer Ebene abbaut (vgl. Askedal 2001). Selbst in diesem exemplarischen Datensatz, der auf Belegen peripherer Kasusmarker aufgebaut ist, die stark zum Synkretismus neigen, gewährleistet die morphologische Kasusdistinktion nach wie vor einen Großteil der strukturellen Disambiguierung der Argumentstruktur. Kasus erweist sich damit sogar hier noch als durchaus funktionsfähige Kategorie. Ein solches ökonomisches Prinzip wurde für Kategorien der (ober)deutschen Morphologie bereits für einen bestimmten syntaktischen Kontext beschrieben:

Das Bedürfnis der Sprecher nach morphologischer Symbolisierung kategorialer Merkmale ist ein relevanter Faktor bei der Ausbildung paradigmatischer Distinktion. Ein morphologisches Minimum wird nicht unterschritten.

(Rabanus 2008: 269) 
Die Feststellung, die Rabanus (2008) für den Ausdruck verschiedener morphologischer Kategorien im Minimalsatz (d. h. in einem Satz bestehend aus einem Verb und seinen obligatorischen, pronominalen Argumenten) trifft, lässt sich auf Grundlage der kurzen, hier angestellten Analyse auch auf die Kasusdistinktion im Syntagma generell übertragen. Betrachtet man ausschließlich die Kasusmorphologie - Rabanus (2008) bezieht in seine Analyse zusätzliche disambiguierende Kategorien wie Kongruenz, Numerus und Person ein -, lässt sich feststellen, dass die paradigmatische, distinkte Markierung im Diasystem größtenteils dort gewährleistet ist, wo sie auf syntagmatischer Ebene zur Disambiguierung der Argumentstruktur notwendig ist, z. B. insbesondere bei Personalpronomen, die als nominale Kasusmarker allein in einer Phrase stehen. Kasussynkretismus kann so mit einer Tendenz zum Abbau von kasusmorphologischen Redundanzen innerhalb der Phrase oder auf Satzebene und damit anhand einer ökonomischen Entwicklung erklärt werden, an deren Ziel die morphologische Distinktion der Argumentstruktur hinreichend, aber nicht redundant ist (vgl. das Konzept des morphologischen Minimums in Rabanus 2008: 262 und 266). Diese Tendenz ist im untersuchten System zwar grundlegend zu erkennen; es sind jedoch trotzdem durchaus sowohl redundante, als auch kasusmorphologisch unterspezifizierte Belege vorhanden.

Letztere, die kasusmorphologisch ambigen Sätze, die zunächst mit diesem Prinzip zu konfligieren scheinen, zeigen jedoch ebenfalls keine vollständige Ambiguität. Beim Großteil dieser Belege (21 Belege, 23\% der synkretischen Akkusativbelege) ist eine kasusmorphologische Disambiguierung der Argumentstruktur nicht notwendig. Hier handelt es sich um intransitive Relationen, um Passivsätze und um prädikative Konstruktionen, innerhalb derer immer ein Subjekt im Nominativ gefordert wird und bei denen das Verb zudem mit eben diesem Subjekt kongruiert, womit eine andere Form der morphologischen Markierung auftritt. (16) illustriert einen solchen Beleg.

$$
\begin{array}{llllll}
\text { on då isch } a & \text { sähr, sähr schlächda } & \text { Luft. } \\
\text {,Und hier ist } & \text { eine_syN } & \text { sehr, sehr } & \text { schlechte_syN Luft. }
\end{array}
$$

Beleg 7977, Alfdorf/Schwäbisch Gmünd

Nur zehn Belege im Transkript zeigen tatsächlich strukturelle Ambiguität. Hier wird die Argumentstruktur weder durch Kasusdistinktion noch durch Kongruenz, somit. durch keine morphologische Kategorie, disambiguiert. Dabei handelt es sich jedoch mit lediglich 11\% um einen äußerst geringen Anteil, der zudem größtenteils durch nicht-morphologische Faktoren disambiguiert ist. Bei acht Belegen zeigt sich eine prototypische Belebtheitsdistinktion: Die synkreti- 
schen Nominalphrasen, die die Adjektivbelege enthalten, sind hier unbelebt (und in einem Fall tierisch) und damit prototypische Objekte, während die Subjekte (die jedoch auch synkretisch sind) ausnahmslos menschlich und damit ebenfalls prototypisch sind (vgl. (17) und (18)).

$$
\begin{array}{lllll}
\text { mãi } & \text { Muader håt koi } & \text { Zeit et gheet, } \\
\text {,Meine_syn } & \text { Mutter } & \text { hat keine_syn } & \text { Zeit nicht gehabt. }
\end{array}
$$

Beleg 7657, Alfdorf/Schwäbisch Gmünd

(18) On mãi Großvadder håt nå ds elderliche ,Und mein_syN Großvater hat dann das elterliche_syN Ãwäsa greddet, Anwesen gerettet.

Beleg 8030, Alfdorf/Schwäbisch Gmünd

Diese semantisch disambiguierten Belege weisen zudem alle die prototypische Abfolge $\mathrm{S}>\mathrm{O}$ auf, sind daher zusätzlich syntaktisch disambiguiert. Bei den einzigen beiden Adjektivbelegen, die hier tatsächlich sowohl als synkretisch klassifiziert wurden als auch in einer strukturell und semantisch ambigen Umgebung auftreten, handelt es sich um Fremdwörter, die vermutlich nicht zum alltäglichen Wortschatz der Gewährsperson gehören (was u. U. eine untypische morphologische Struktur begünstigt) und die zudem keine Argumente, sondern Adjunkte ${ }^{117}$ ausdrücken (vgl. (19)).

Ond ous higenische Grend därfa då
,Und aus hygienischen_sYN Gründen dürfen hier
koi $\quad$ Schtonda abghalda wärra.
keine_syN
Stunden abgehalten werden.

Beleg 8051, Alfdorf/Schwäbisch Gmünd

Die beispielhafte Analyse konnte damit erste Erkenntnisse zur Struktur der syntagmatischen Kasusmarkierung und zum Einfluss syntagmatischer Strukturen auf das Kasusparadigma liefern: Syntagmatische und paradigmatische Kasusmarkierung zeigen eine interessante Interaktion, die es vertiefend $\mathrm{zu}$ untersuchen gilt. Es zeichnet sich ab, dass (prototypische) Positionen beziehungsweise

117 Im hier angeführten Beispiel kann angelehnt an Blake (2001: 9) zum Lateinischen davon ausgegangen werden, dass der Dativ - zumindest grundsätzlich - durch die Präposition, die im Deutschen immer Dativ fordert, eindeutig markiert ist. 
Funktionen im Syntagma Auswirkungen auf die Distinktionsquote der Kasusmarker haben. Das Diasystem lässt paradigmatischen Synkretismus eher bei den Kasusmarkern zu, die innerhalb der Phrase bzw. innerhalb des Satzes häufig durch weitere Kasusmarker ergänzt werden (z. B. Adjektive und Determinierer), während solche, die eher alleine stehen (z. B. Personalpronomen), eher Distinktion zeigen. Dieser Zusammenhang konnte in der Analyse erstmals quantifiziert und zudem unter Einbeziehen des Kontexts sowie von semantischen und syntaktischen Kategorien in gesprochensprachlichen Daten analysiert werden.

Die Analyse hat sich jedoch auf einen beschränkten Datensatz gestützt. Innerhalb eines einzelnen (rekonstruierten) Idiolekts wurden lediglich die Sätze analysiert, die Adjektive enthalten. In vertiefenden Analysen können auch weitere Kasusmarker, wie beispielsweise Definit- und Indefinitartikel, die hier lediglich in Abhängigkeit zum Adjektiv aufgeführt wurden, auf syntagmatische Interaktionen hin untersucht werden. Eine breitere Datengrundlage, die durchaus auch auf Basis von Ruoff (1984) erstellt werden könnte, würde außerdem erlauben, geographische Analysen auch bezüglich der Kasusdistinktion im Syntagma anzustellen und so zu überprüfen, inwiefern sich die Unterschiede, die in 4.3 für die maskulinen Kategorien auf paradigmatischer Ebene beschrieben wurden, auch in den syntagmatischen Strukturen spiegeln.

Einen Anhaltspunkt dafür, dass sich auf Ebene des Syntagmas geographische Unterschiede zeigen können, liefern die Ergebnisse der Untersuchung von Baechler/Pröll (2018), die sich einer ähnlichen Fragestellung - nämlich der nach der „Kompensation“ von Kasusnivellierung am Nomen durch Artikel und Adjektive innerhalb einer Nominalphrase - u. a. mit höchstalemannischen Daten widmen. Dort findet sich eine distinkte Markierung im Syntagma innerhalb der Nominalphrase lediglich beim Dativ, wo es sogar zu syntagmatischen kasusmorphologischen Redundanzen kommt. Nominativ und Akkusativ sind in diesem Gebiet, das auch im Maskulinum zum Muster NA/D neigt, sowohl paradigmatisch als auch syntagmatisch synkretisch (Baechler/Pröll 2018: 20-21). Eine Interaktion von paradigmatischer und syntagmatischer Kasusmarkierung wie in der hier vorgestellten Korpusanalyse kann dort nicht festgestellt werden.

Dieser Unterschied kann durch die geographische Lage und die damit einhergehenden Unterschiede in den Mustern der Kasusmarkierung vermutet werden. Das hier beispielhaft untersuchte Teilsystem aus Alfdorf/Schwäbisch Gmünd entstammt dem Kerngebiet der vollen Distinktion, in dem Nominativ und Akkusativ auch im Maskulinum distinkt sind, wo demnach grundsätzlich mehr Möglichkeiten zur paradigmatischen Kasusdistinktion vorhanden sind als im von Baechler/ Pröll (2018) untersuchten Gebiet.

Neben den abweichenden paradigmatischen Voraussetzungen des Untersuchungsgebiets in Baechler/Pröll (2018) unterscheidet sich diese Arbeit auch von 
der hier angestellten Analyse jedoch in der genutzten Datenbasis. Baechler/Pröll (2018) beziehen sich auf Paradigmen aus Orts- und Dialektgrammatiken und rekonstruieren die Nominalphrasen aus den dort aufgeführten Paradigmen zu Nomen, Artikeln und Adjektiven. Ein Vergleich der Erkenntnisse zur Interaktion verschiedener Phrasen auf Satzebene, die im Rahmen der Korpusanalyse gewonnen werden konnten und der sich in Anbetracht der unterschiedlichen Ergebnisse angeboten hätte, ist daher nicht möglich. Auch Baechler/Pröll (2018: 26) gehen jedoch von einer gewissen Interaktion der (synkretischen) Nominalphrasen mit syntagmatischen Strukturen zur Disambiguierung der Argumentstruktur aus und verweisen mit Werlen (1990) auf eine Tendenz zur festen Wortfolge (S > 0) in alemannischen Dialekten.

Die Unterschiede zu den Ergebnissen von Baechler/Pröll (2018) stellen in jedem Fall einen guten Ausgangspunkt für vertiefende, geographisch vergleichende Untersuchungen syntagmatischer Kasusmarkierung dar, die auf Grundlage der hier skizierten Methode angestellt werden können.

Die hier beispielhaft durchgeführte Analyse zur Interaktion von Kasusmarkierung in Paradigma und Syntagma kann die Aussagen von Dal (1971b: 177) und Dürscheid (2007: 110) zum Status der Kategorie Kasus im Hochdeutschen abschließend grundlegend bestätigen: Die Nivellierungstendenzen scheinen - zumindest im hier untersuchten Teilsystem - primär bestimmte morphologische Marker zu betreffen; die Kategorie Kasus und ihr distinkter Ausdruck auf syntagmatischer Ebene scheint davon jedoch nicht so stark beeinflusst zu sein, dass ihre Grundfunktion davon in größerem Umfang beeinträchtigt ist. Neben verschiedenen anderen, wie beispielsweise Kongruenz, Serialisierung und Belebtheit, stellt Kasus im untersuchten System einen Mechanismus zur Markierung der Argumentstruktur dar, der diese Funktion in einem Großteil der Kontexte erfüllt. Kasus kann damit als durchaus funktionsfähige Kategorie im untersuchten Diasystem bewertet werden. 